\title{
Morse Homotopy and Chern-Simons Perturbation Theory
}

\section{Kenji Fukaya $^{1}$}

Department of Mathematics, Faculty of Sciences, Kyoto University, Kitashirakawa-Oiwake-cho, Sakyo-ku, Kyoto, Japan E-mail: fukaya@kusm kyoto-u ac.jp

Received: 1 June 1995/Accepted: 2 February 1996

\begin{abstract}
We define an invariant of a three manifold equipped with a flat bundle with vanishing homology. The construction is based on Morse theory using several Morse functions simultaneously and is regarded as a higher loop analogue of various product operations in algebraic topology. There is a heuristic argument that this invariant is related to perturbative Chern-Simons Gauge theory by Axelrod-Singer, etc. There is also a theorem which gives a relation of the construction to open string theory on the cotangent bundle.
\end{abstract}

\section{Contents}

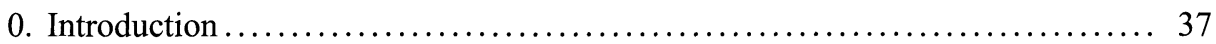

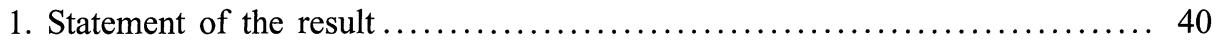

2. Transversality and compactness $\ldots \ldots \ldots \ldots \ldots \ldots \ldots \ldots \ldots \ldots \ldots \ldots \ldots \ldots \ldots, 48$

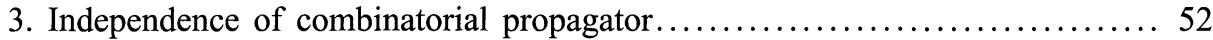

4. Independence of the Morse functions I ............................. 59

5. Independence of the Morse functions II ........................... 62

6. Compactification of configuration space and transversality at diagonal ..... 73

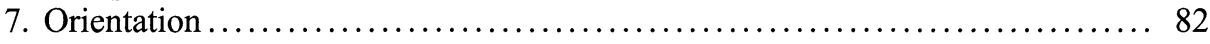

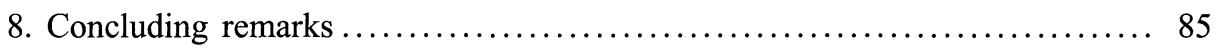

\section{Introduction}

Let $M$ be a $2 n$ dimensional manifold and $N$ be its $n$ dimensional submanifold. We consider a current $T_{N}$ such that $T_{N}(\omega)=\int_{N} \omega$. We try to justify the integral

$$
\int_{M} T_{N} \wedge T_{N}
$$

(We remark that $T_{N} \wedge T_{N}$ itself is not well defined.) One way to do so is to take a perturbation $N^{\prime}$ of $N$ so that $N^{\prime}$ and $N$ are transversal to each other and consider

\footnotetext{
${ }^{1}$ Partially supported by Grants-in-Aid for Scientific Research on Priority Areas 231 "Infinite Analysis"
} 
$\int_{M} T_{N} \wedge T_{N^{\prime}}$. It is easy to see that $T_{N} \wedge T_{N^{\prime}}$ is a delta current supported at the intersection $N \cap N^{\prime}$. Therefore $\int_{M} T_{N} \wedge T_{N^{\prime}}$ is the intersection number $N \bullet N^{\prime}=$ $N \bullet N$. What is important here is that the integral $\int_{M} T_{N} \wedge T_{N^{\prime}}$ is independent of the perturbation $N^{\prime}$.

There is an alternative way to get the same answer. Namely we choose a harmonic $n$ form $h_{N}$ which represents the De-Rham cohomology class of $N$. Then again $\int_{M} h_{N} \wedge h_{N}$ is well defined and gives the self intersection number $N \bullet N$.

We can continue in a similar way to define the secondary invariant as follows. Let $M=\Delta \subseteq M^{2}$ be the diagonal. Assume that $T_{\Delta}$ is an exact current. (In fact this never happens. But we can find this kind of situation by working with a local coefficient.) We choose an $n-1$ form $\omega$ on $M^{2}$ such that $d \omega=T_{\Delta}$. Assume that $M$ is three dimensional. Then the integral

$$
\int_{M^{2}} \omega \wedge \omega \wedge \omega
$$

is a number. By modifying this integral $\int_{M^{2}} \omega \wedge \omega \wedge \omega$, an invariant of a 3 manifold is discovered by Axelrod-Singer [AS], Bar-Natan [Ba], Guadagnini-MartinelliMintchev [GMM], Kontsevich [Ko], etc. We discuss their result a bit more in Sect. 8. Their construction is based on harmonic theory and hence is an analogy of the second method we mentioned above to justify $(0.1)$.

On the other hand, we can also imitate the first approach. Namely we can use an appropriate intersection number to justify $(0.2)$. To perform this kind of construction is the purpose of this paper.

To do so, we need to find a cycle $X$ such that $\partial X=\Delta$. Such an $X$ can be found as follows. Choose a Morse function $f$. Let $M(f)$ be the set of all pairs $(x, y) \in M^{2}$ such that $x, y$ lie on the same gradient line of $f$. One can easily find that a connected component of the boundary of $M(f)$ is a diagonal $\Delta$. Hence the intersection number $M\left(f_{1}\right) \bullet M\left(f_{2}\right) \bullet M\left(f_{3}\right)$ should be related to (0.2).

Let us here review various results related to the contents of this paper. First it was discovered by [As, Ba, GMM, Ko] that an integral like $\int_{M^{2}} \omega \wedge \omega \wedge \omega$ gives the second term of the expansion of Witten's invariant [W2, Kh, ReT] that is a ChernSimons gauge theory. The first term of this expansion is basically the Analytic torsion of Ray-Singer and was discussed by Witten [W2]. Axelrod-Singer, etc. defined a higher term also.

In fact it is not yet proved that the construction of Axelrod-Singer, etc. really gives the expansion. What they did is to give an argument of physical level of rigor to show that this is an expansion of the Witten invariant and also they proved rigorously that the invariant is independent of the various choices involved.

Witten in [W3] found that Chern-Simons Gauge theory on a 3-manifold $M$ is equivalent to the open string field theory of its cotangent bundle $T^{*} M$. However to make the latter rigorous in a mathematical sense is not easy and remains yet an open question. (See Sect. 8 for more discussion about it.)

Floer studied the pseudo-holomorphic disk with Lagrangian boundary condition to define his celebrated Floer homology for Lagrangian intersection [F11]. In the case of the cotangent bundle he proved that Floer homology between 0 section and its Hamiltonian perturbation is equal to the usual homology of the manifold. The problem to define Floer homology for Lagrangian intersection in a more general situation is studied by $\mathrm{Oh}$ [Oh]. The author introduced an $A^{\infty}$-structure on Floer homology [Fu1, Fu2]. In the case of the cotangent bundle $T^{*} M$, it is proved by 
$\mathrm{Oh}$ and the author that the $A^{\infty}$-structure of Floer homology is equivalent to one for $M$ [FO]. The latter is described by using Morse theory. The main idea to do so is to use several Morse functions at once. This idea is due to the author [Fu2, Fu3] and to $\mathrm{M}$. Betz and R. Cohen [BC] independently. Roughly speaking we consider the moduli space of maps from a graph to $M$ such that each edge is a gradient line of some Morse function. The $A^{\infty}$-structure then corresponds to the case when the graph is a tree. The result of $\mathrm{Oh}$ and the author says that the case of a tree is equivalent to the 0 -loop amplitude of open string field theory on $T^{*} M$.

Betz-Cohen studied also the graph which is not a tree. They announced that characteristic classes of a manifold are described in that way. The author in [Fu3] also studied the case when the graph is not a tree. However the discussion there was not yet satisfactory.

The main point of this paper is that the Morse theory for a graph which is not a tree gives Chern-Simons perturbation theory. The author does not yet prove that they really coincide. What is proved in this paper is that (for the 2 loop amplitude) there is a well defined invariant based on Morse theory.

We can generalize our result with $\mathrm{Oh}$ to this case and show that our invariant is related to open string theory (see Sect. 8). Thus in a sense our construction is a rigorous mathematical definition of a 2 loop open string amplitude on the cotangent bundle of a 3 manifold.

It is remarkable that our construction is similar to the construction of ChernSimons Perturbation theory in many points. Also in Sect. 8 we discuss some heuristic argument which suggests that our invariant coincides to Chern-Simons Perturbation theory. This idea is closely related to Witten's work on Morse theory [W1].

Note that the invariant we introduced here is a secondary invariant to the (co)homology group (with cup product). Also here the homology group is studied from the point of view of singular theory (since Morse function gives a cell decomposition.) With this respect Reidemeister torsion is another natural secondary invariant of the (co)homology theory with local coefficient. In Chern-Simons perturbation theory analytic torsion of Ray-Singer [RS] appeared as the first invariant. The coincidence of Reidemeister and Analytic torsion was established by Cheeger [Ch] and Müller [Mü]. Our conjecture that the invariant in this paper coincides with one by Axelrod-Singer, etc. may be regarded as the higher genus analogue of this theorem of Cheeger-Müller.

The organization of this paper is as follows:

In Sect. 1 we define our invariant. Roughly speaking it is obtained by counting the order of the set of solutions of an appropriate ordinary differential equation, with appropriate weight.

The proof that this number is independent of various choices is based on the study of compactification of the moduli space of the solution of an ordinary differential equation. There are basically two points to clarify. To define an intersection number $M\left(f_{1}\right) \bullet M\left(f_{2}\right) \bullet M\left(f_{3}\right)$, there are two problems. One is that the boundary of $M\left(f_{1}\right)$ is not in fact equal to the diagonal $\Delta$. There is another boundary that is the set of pairs $(x, y)$, where $x$ is in an unstable manifold of grad $f$ with respect to a critical point $p$ of $f$ and $y$ is in the stable manifold of the same critical point $p$. We need to add various correction terms to settle this problem.

In Sect. 2, we discuss the point mainly related to this problem.

Based on it the well-definedness of our invariant is proved in Sects. 3-5. 
In Sect. 6, we discuss another problem with the transversality of $M\left(f_{1}\right) \bullet M\left(f_{2}\right) \bullet$ $M\left(f_{3}\right)$. Namely these submanifolds are not transversal to each other at the diagonal, even if we choose $f_{i}$ generic. We need to use an appropriate blow up of the diagonal of $M^{2}$. This construction is similar to the argument of compactification of configuration space due to Fulton-Macpherson [FM] and Kontsevich [Ko], which was used also by [AS] and [Ko].

In Sect. 7 we discuss orientation of our moduli space.

In Sect. 8 we describe some ideas related to the problem that our invariant is equal to both Chern-Simons perturbation theory and open string theory of the cotangent bundle.

The result of this paper was announced in [Fu4] without proof.

During the preparation of this paper, the author visited Maryland University, Hong Kong University of Science and Technology, Newton Institute, International Center of Theoretical Physics and Stanford University. The author would like to thank these universities and institutes for their hospitalities.

The author would also like to thank Professor Hiraku Nakajima for his excellent explanation of Chern-Simons perturbation theory to the author. He also would like to thank T. Gocho who pointed out an error on sign in the preliminary version.

\section{Statement of the Result}

Let $M$ be a compact oriented 3 manifold and $\xi$ be a flat vector bundle on it. In this section we define a number $Z_{2}\left(M ; f_{1}, f_{2}, f_{3} ; \xi\right)$ by fixing a metric on $M$ and three generic functions $f_{1}, f_{2}, f_{3}$ on $M$. The proof that it is invariant of these choices will be given in Sects. $2-7$.

The number $Z_{2}\left(M ; f_{1}, f_{2}, f_{3} ; \xi\right)$ is a sum of $\hat{Z}_{\Theta}\left(M ; f_{1}, f_{2}, f_{3} ; \xi\right)$ and correction terms. We first define the leading term.

For a function $f$ on $M$, let $\Phi_{f}^{t}: M \rightarrow M$ be the one parameter group of diffeomorphisms associated to grad $f$. Namely it satisfies

$$
\left\{\begin{array}{l}
\Phi_{f}^{0}(x)=x \\
\left.\frac{\partial \Phi_{f}^{t}(x)}{\partial t}\right|_{t=t_{0}}=\operatorname{grad} f\left(\Phi_{f}^{t_{0}}(x)\right) .
\end{array}\right.
$$

Let $f_{1}, f_{2}, f_{3} \in M$. We put

$$
\mathscr{M}_{\Theta}\left(f_{1}, f_{2}, f_{3}\right)=\left\{\left(x, y ; t_{1}, t_{2}, t_{3}\right) \in M^{2} \times \mathbf{R}_{+}^{3} \mid \Phi_{f_{i}}^{t_{i}}(x)=y, i=1,2,3\right\} .
$$

Lemma 1.2. For generic $f_{1}, f_{2}, f_{3}$, the space $\mathscr{M}_{\Theta}\left(f_{1}, f_{2}, f_{3}\right)$ is an oriented manifold of dimension $3-\operatorname{dim} M$.

Lemma 1.3. If $\operatorname{dim} M=3$, then for generic $f_{1}, f_{2}, f_{3}$, the space $\mathscr{M}_{\Theta}\left(f_{1}, f_{2}, f_{3}\right)$ is compact.

These lemmas are proved in Sect. 2.

For $t_{1}, t_{2}, t_{3}>0$, we define a metric on $\Theta$ as follows. Let $e_{j}, j=1,2,3$ be the edges of length $t_{1}, t_{2}, t_{3}$ respectively. We take two vertices $v_{1}, v_{2}$ and attach each of the edges of $e_{j}$ to $v_{1}, v_{2}$. 


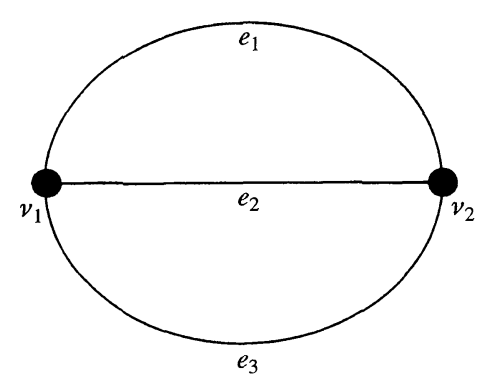

Fig. 1 .

For an element $\left(x, y ; t_{1}, t_{2}, t_{3}\right)$ of $\mathscr{M}_{\Theta}\left(f_{1}, f_{2}, f_{3}\right)$ we associate a map $I: \Theta \rightarrow M$ as follows. We put $I\left(v_{1}\right)=x, I\left(v_{2}\right)=y$, and on the edge $e_{j}$ we define $I(t)=$ $\Theta_{f_{j}}^{t}(x)$. (Here we identify $e_{j}=\left[0, t_{j}\right]$.) By the definition of $\mathscr{M}_{\Theta}\left(f_{1}, f_{2}, f_{3}\right)$ this map is continuous at $v_{2}$. Hereafter we regard an element of $\mathscr{M}_{\Theta}\left(f_{1}, f_{2}, f_{3}\right)$ as such a map.

We next associate a weight $\chi(I, \xi)$ to each element $I: \Theta \rightarrow M$ and a flat vector bundle $\xi$ as follows. We define element $\gamma_{i} \in \pi_{1}(\Theta), i=1,2,3,4$ by

$$
\begin{aligned}
& \gamma_{1}=e_{2}^{-1} \circ e_{1}, \\
& \gamma_{2}=e_{3}^{-1} \circ e_{2}, \\
& \gamma_{3}=e_{1}^{-1} \circ e_{3}, \\
& \gamma_{4}=e_{2}^{-1} \circ e_{3} \circ e_{1}^{-1} \circ e_{2} \circ e_{3}^{-1} \circ e_{1} .
\end{aligned}
$$

Roughly speaking, $\gamma_{1}, \gamma_{2}, \gamma_{3}$ are boundary loops of one of the ribbon structures of the $\Theta$-graph, while $\gamma_{4}$ is the boundary loop of another ribbon structure of the $\Theta$-graph. Now we put

$$
\chi(I, \xi)=-\prod_{i=1}^{3} \operatorname{Tr}\left(\operatorname{Hol}_{\xi}\left(I_{*}\left(\gamma_{i}\right)\right)+\operatorname{Tr}\left(\operatorname{Hol}_{\xi}\left(I_{*}\left(\gamma_{4}\right)\right)\right.\right.
$$

Here $\operatorname{Hol}_{\xi}(\ell)$ is the holonomy homomorphism of the flat bundle $\xi$ along the loop $\ell$. Now we define

\section{Definition 1.5.}

$$
Z_{\Theta}\left(f_{1}, f_{2}, f_{3} ; \xi\right)=\sum_{I \in \mathscr{M}_{\Theta}\left(f_{1}, f_{2}, f_{3}\right)} \varepsilon_{I} \chi(I, \xi)
$$

Before going to the next step, let us rewrite our weight by using Lie algebra bundle $\varsigma=\operatorname{Ad} \xi$. More generally we consider a flat bundle $\varsigma$ over $M$, whose fibre has a structure of semi-simple Lie algebra compatible with the flat structure. By using a canonical invariant inner product, we have an inner product on $\varsigma$ which is also compatible with the flat structure. Let $I \in \mathscr{M}_{\Theta}\left(f_{1}, f_{2}, f_{3}\right)$. We choose an orthonormal basis $\mathbf{e}_{1}, \ldots, \mathbf{e}_{\operatorname{dim} g}$ of $\varsigma_{x}$ for $x=I\left(v_{1}\right)$. We put

$$
\chi(I, \varsigma)=\sum_{i, j, k=1}^{\operatorname{dim} g}\left\langle\left[\mathbf{e}_{i}, \mathbf{e}_{j}\right], \mathbf{e}_{k}\right\rangle\left\langle\left[P_{e_{1}}\left(\mathbf{e}_{i}\right), P_{e_{3}}\left(\mathbf{e}_{k}\right)\right], P_{e_{2}}\left(\mathbf{e}_{j}\right)\right\rangle .
$$

Here $P_{e_{i}}: \varsigma_{x} \rightarrow \varsigma_{y}$ is the parallel transport along the path $I\left(e_{i}\right)$. 
Lemma 1.7. If $\xi$ is a $\mathbf{C}^{2}$ bundle with flat $\operatorname{su}(2)$ structure and if $\varsigma=\operatorname{Ad} \xi$, then we have

$$
2 \chi(I, \xi)=\chi(I, \varsigma) .
$$

Proof. We embed $\mathrm{su}(2) \rightarrow \operatorname{gl}(2 ; \mathbf{C})$ and choose $\mathbf{e}_{0}$ so that $\mathbf{e}_{0}, \ldots, \mathbf{e}_{3}$ is an orthonormal basis of $\operatorname{gl}(2 ; \mathbf{C})$ and $\mathbf{e}_{1}, \mathbf{e}_{2}, \mathbf{e}_{3}$ is an orthonormal basis of $\operatorname{su}(2)$. Then since $\mathbf{e}_{0}$ is in the center, we have

$$
\chi(I, \varsigma)=\sum_{i, j, k=0}^{3}\left\langle\left[\mathbf{e}_{i}, \mathbf{e}_{j}\right], \mathbf{e}_{k}\right\rangle\left\langle\left[P_{e_{1}}\left(\mathbf{e}_{i}\right), P_{e_{3}}\left(\mathbf{e}_{k}\right)\right], P_{e_{2}}\left(\mathbf{e}_{j}\right)\right\rangle .
$$

We then find that $(1.8)$ is equal to

$$
\begin{aligned}
& -2 \sum_{i, j, k=0}^{3}\left\langle\mathbf{e}_{i} \mathbf{e}_{j}, \mathbf{e}_{k}\right\rangle\left\langle P_{e_{1}}\left(\mathbf{e}_{i}\right) P_{e_{2}}\left(\mathbf{e}_{j}\right), P_{e_{3}}\left(\mathbf{e}_{k}\right)\right\rangle \\
& +2 \sum_{i, j, k=0}^{3}\left\langle\mathbf{e}_{i} \mathbf{e}_{j}, \mathbf{e}_{k}\right\rangle\left\langle P_{e_{2}}\left(\mathbf{e}_{j}\right) P_{e_{1}}\left(\mathbf{e}_{i}\right), P_{e_{3}}\left(\mathbf{e}_{k}\right)\right\rangle .
\end{aligned}
$$

(Here $\mathbf{e}_{i} \mathbf{e}_{j}$ etc. denotes the product of matrix.)

It is straightforward to see that (1.9) is independent of the choice of orthonormal basis. So we change our basis and take $\mathbf{f}_{i} \otimes \mathbf{f}^{j}, i, j=1,2$. Here $\mathbf{f}_{1}, \mathbf{f}_{2}$ is an orthonormal basis of $\xi_{x}$ and $\mathbf{f}^{1}, \mathbf{f}^{2}$ is its dual basis. Using this basis the first term of (1.9) is

$$
\begin{aligned}
& 2 \sum_{i, j, k=1}^{2}\left\langle\left(\mathbf{f}_{i} \otimes \mathbf{f}^{j}\right)\left(\mathbf{f}_{j} \otimes \mathbf{f}^{k}\right),\left(\mathbf{f}_{k} \otimes \mathbf{f}^{i}\right)\right\rangle\left\langle P_{e_{1}}\left(\mathbf{f}_{i} \otimes \mathbf{f}^{j}\right) P_{e_{2}}\left(\mathbf{f}_{j} \otimes \mathbf{f}^{k}\right), P_{e_{3}}\left(\mathbf{f}_{k} \otimes \mathbf{f}^{i}\right)\right\rangle \\
& \quad=2 \sum_{i, j, k=1}^{2}\left\langle P_{e_{1}}\left(\mathbf{f}_{i}\right), P_{e_{3}}\left(\mathbf{f}_{i}\right)\right\rangle\left\langle P_{e_{1}}\left(\mathbf{f}_{j}\right), P_{e_{2}}\left(\mathbf{f}_{j}\right)\right\rangle\left\langle P_{e_{2}}\left(\mathbf{f}_{k}\right), P_{e_{3}}\left(\mathbf{f}_{k}\right)\right\rangle . \\
& \quad=2 \prod_{i=1}^{3} \operatorname{Tr}\left(\operatorname{Hol}_{\xi}\left(\gamma_{i}\right)\right) .
\end{aligned}
$$

Similarly we find that the second term of (1.9) is equal to $2 \operatorname{Tr}\left(\operatorname{Hol}_{\xi}\left(\gamma_{4}\right)\right)$. The proof of Lemma 1.7 is now complete.

We define

$$
Z_{\Theta}\left(f_{1}, f_{2}, f_{3} ; \varsigma\right)=\sum_{I \in \mathscr{M}_{\Theta}\left(f_{1}, f_{2}, f_{3}\right)} \pm \chi(I, \varsigma)
$$

for a flat Lie algebra bundle $\varsigma$. Here \pm is determined by the orientation of $\mathscr{M}_{\Theta}\left(f_{1}, f_{2}, f_{3}\right)$, which is discussed in Sect. 7 .

We put

$$
\tilde{Z}_{\Theta}\left(f_{1}, f_{2}, f_{3} ; \varsigma\right)=\frac{1}{8} \sum_{\varepsilon_{1}= \pm 1} Z_{\Theta}\left(\varepsilon_{1} f_{1}, \varepsilon_{2} f_{2}, \varepsilon_{3} f_{3} ; \varsigma\right) .
$$

We thus defined the leading term of our invariant. To define correction terms, we first introduce the Witten complex [W1] with local coefficient. Let $f: M \rightarrow \mathbf{R}$ be a Morse function. We put

$$
C_{k}(M ; f ; \varsigma)=\bigoplus_{\substack{p \in \operatorname{Cr}(f) \\ \eta(p)=k}} \varsigma_{p} .
$$


Here $\operatorname{Cr}(f)$ is the set of critical points of $f$ and $\eta(p)$ is its Morse index. We define the boundary operator $\partial: C_{k}(M ; f ; \varsigma) \rightarrow C_{k-1}(M ; f ; \varsigma)$ as follows. Let $\eta(p)=\eta(q)+1$. We are going to define the component $\partial_{p q}: \varsigma_{p} \rightarrow \varsigma_{q}$ of $\partial$. Following the definition of the usual Witten complex, we use the moduli space

$$
\mathscr{M}(p, q)=\left\{\begin{array}{l|l}
\ell: \mathbf{R} \rightarrow M & \begin{array}{l}
\frac{d \ell}{d t}=-\operatorname{grad} f \\
\lim _{\substack{t \rightarrow-\infty \\
\lim _{t \rightarrow+\infty} \ell(t)=p}} \ell(t)=q
\end{array}
\end{array}\right\} .
$$

In case the Morse function $f$ is generic and $\eta(p)=\eta(q)+1$, this space is an oriented manifold of dimension one. The group $\mathbf{R}$ acts on it by translation of parameter. Let $\overline{\mathscr{M}}(p, q)$ be the quotient space of this action. We then define $\partial_{p q}: \varsigma_{p} \rightarrow \varsigma_{q}$ by

$$
\partial_{p q}=\sum_{\ell \in \bar{M}(p, q)} \pm P_{\ell}
$$

Here $P_{\ell}$ is the parallel transportation along the arc $\ell$, and the sign is determined by the orientation of the moduli space $\overline{\mathscr{M}}(p, q)$.

Lemma 1.11. $\partial \partial=0, H_{*}\left(C_{*}(M ; f ; \varsigma), \partial\right)=H_{*}(M ; \varsigma)$.

Here the right-hand side is the homology with local coefficient. The proof of Lemma 1.11 is a straightforward analogue of the result in the case when $\varsigma$ is trivial. The proof in that case is given by various authors. See for example [Sc].

Now we go back to our problem to define correction terms. We make use of the assumption $H(M ; \varsigma)=0$. By Lemma 1.11 it follows that the chain complex $\left(C_{*}(M ; f ; \varsigma), \partial\right)$ is acyclic. Hence so is $\operatorname{End}\left(C_{*}(M ; f ; \varsigma), \partial\right)$. The element $i d \in \operatorname{End}\left(C_{*}(M ; \varsigma), \partial\right)$ is a cycle. So there exists an element $g_{f, \varsigma} \in \operatorname{End}\left(C_{*}(M ; f ; \varsigma)\right)$ of degree one such that $\partial g_{f, \varsigma}=i d$. (Namely $\partial \circ g_{f, \varsigma}+g_{f, \varsigma} \circ \partial=i d$.) We call such $g_{f, \varsigma}$ a combinatorial propagator. We remark that combinatorial propagator is not unique. But we prove later that the invariant we define using it is independent of the choice of combinatorial propagator.

Now let $c_{i} \in \operatorname{End}_{l}\left(C_{*}\left(M ; f_{i} ; \varsigma\right)\right)$. We are going to define $Z_{\Theta}\left(c_{1}, \varnothing, \varnothing ; f_{1}, f_{2}\right.$, $\left.f_{3} ; \varsigma\right)$ etc. We put

$$
c_{1}=\sum_{\eta(p)=\eta(q)+1} c(p, q)[p] \otimes[q]^{*} .
$$

Here $c(p, q) \in \operatorname{Hom}\left(\varsigma_{q}, \varsigma_{p}\right)$. For $\eta(p)=\eta(q)+1$, we define a moduli space $\mathscr{M}_{\Theta(1,0,0)}\left(p, q ; f_{1}, f_{2}, f_{3}\right)$ as follows:

$$
\mathscr{M}_{\Theta(1,0,0)}\left(p, q ; f_{1}, f_{2}, f_{3}\right)=\left\{\begin{array}{l|l}
\left(x, y ; t_{2}, t_{3}\right) \in M^{2} \times \mathbf{R}_{+}^{2} & \begin{array}{l}
\lim _{t \rightarrow+\infty} \Phi_{f_{1}}^{t}(x)=p \\
\lim _{t \rightarrow-\infty} \Phi_{f_{1}}^{t}(y)=q \\
\Phi_{f_{i}}^{t_{2}}(x)=y, i=2,3
\end{array}
\end{array}\right\} .
$$

As in the previous case, an element of the moduli space $\mathscr{M}_{\Theta(1,0,0)}\left(p, q ; f_{1}, f_{2}, f_{3}\right)$ can be regarded as a map from a graph to $M$. Namely let $\Theta(1,0,0)$ be the graph obtained by cutting the first edge $e_{1}$ of the $\Theta$-graph. Then $\Theta(1,0,0)$ has 4 edges, $e_{1}^{1}, e_{1}^{2}, e_{2}, e_{3}$. For an element $\left(x, y ; t_{2}, t_{3}\right)$, we let $e_{1}^{1}$ be mapped to the gradient line of $f_{1}$ joining $x$ to $p, e_{1}^{2}$ is mapped to the gradient line of $f_{1}$ joining $q$ to $y . e_{2}, e_{3}$ will be mapped to the gradient line of $f_{2}, f_{3}$ joining $x$ to $y$ respectively. 
Let $I: \Theta(1,0,0) \rightarrow M$ be a map identified to an element of $\mathscr{M}_{\Theta(1,0,0)}\left(p, q ; f_{1}\right.$, $\left.f_{2}, f_{3}\right)$. We define a weight $\chi(I, \varsigma) \in \operatorname{Hom}\left(\varsigma_{p}, \varsigma_{q}\right)$ as follows. Let $u \in \varsigma_{p}, v \in \varsigma_{q}$. Let $\mathbf{e}_{i}$ be an orthonormal basis of $\varsigma_{x}$. We then put:

$$
\langle\chi(I, \varsigma)(u), v\rangle=\sum_{j, k}\left\langle\left[P_{e_{1}^{1}}(u), \mathbf{e}_{j}\right], \mathbf{e}_{k}\right\rangle\left\langle\left[P_{e_{1}^{2}} v, P_{e_{3}} \mathbf{e}_{k}\right], P_{e_{2}}\left(\mathbf{e}_{j}\right)\right\rangle .
$$

Now we define

$$
Z_{\Theta}\left(c, \varnothing, \varnothing ; f_{1}, f_{2}, f_{3} ; \varsigma\right)=\sum_{I \in \mathscr{M}_{\Theta(1,0,0)}\left(p, q ; f_{1}, f_{2}, f_{3}\right)} \pm \operatorname{Tr}(c(p, q) \circ \chi(I, \varsigma)) .
$$

Here the sign is defined by making use of the orientation of the moduli space. (see Sect. 7.) Using it we define

$$
\begin{aligned}
\hat{\bar{Z}}_{\Theta}\left(f_{1}, f_{2}, f_{3} ; \varsigma\right)= & Z_{\Theta}\left(f_{1}, f_{2}, f_{3} ; \varsigma\right)-Z_{\Theta}\left(g_{f_{1}}, \varnothing, \varnothing ; f_{1}, f_{2}, f_{3} ; \varsigma\right) \\
& -Z_{\Theta}\left(\varnothing, g_{f_{2}}, \varnothing ; f_{1}, f_{2}, f_{3} ; \varsigma\right)-Z_{\Theta}\left(\varnothing, \varnothing, g_{f_{3}} ; f_{1}, f_{2}, f_{3} ; \varsigma\right) \\
& +Z_{\Theta}\left(g_{f_{1}}, g_{f_{2}}, \varnothing ; f_{1}, f_{2}, f_{3} ; \varsigma\right)+Z_{\Theta}\left(g_{f_{1}}, \varnothing, g_{f_{3}} ; f_{1}, f_{2}, f_{3} ; \varsigma\right) \\
& +Z_{\Theta}\left(\varnothing, g_{f_{2}}, g_{f_{3}} ; f_{1}, f_{2}, f_{3} ; \varsigma\right)-Z_{\Theta}\left(g_{f_{1}}, g_{f_{2}}, g_{f_{3}} ; f_{1}, f_{2}, f_{3} ; \varsigma\right) .
\end{aligned}
$$

We then put

$$
\hat{Z}_{\Theta}\left(f_{1}, f_{2}, f_{3} ; \varsigma\right)=\frac{1}{4} \sum_{\varepsilon_{1}= \pm 1} \hat{\bar{Z}}_{\Theta}\left(\varepsilon_{1} f_{1}, \varepsilon_{2} f_{2}, \varepsilon_{3} f_{3} ; \varsigma\right)
$$

(See Remark 1.17 the reason why we take $1 / 4$ in place of $1 / 8$.) The numbers defined in (1.14), (1.15) may depend on the choice of combinatorial propagators.

To obtain a number independent of the choice of functions $f_{i}$ and the Riemannian metric $g$ of $M$, we need to do a similar construction for the graph $\Lambda$ in Fig. 3. We first consider

$$
\mathscr{M}_{\Lambda}\left(f_{1}, f_{2}, f_{4}\right)=\left\{\begin{array}{l|l}
\left(x, y ; t_{1}, t_{2}, t_{4}\right) \in M^{2} \times \mathbf{R}_{+}^{3} & \begin{array}{l}
\Phi_{f_{1}}^{t_{1}}(x)=x \\
\Phi_{f_{2}}^{t_{2}}(y)=y \\
\Phi_{f_{4}}^{t_{4}}(x)=y
\end{array}
\end{array}\right\} .
$$

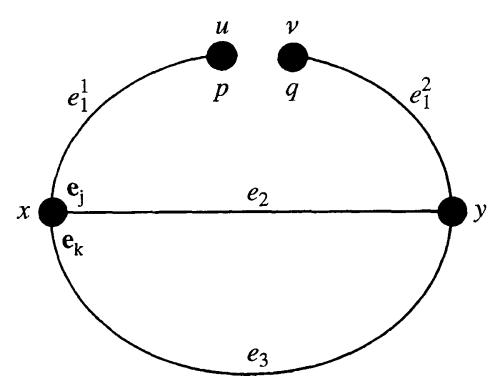

Fig. 2. 


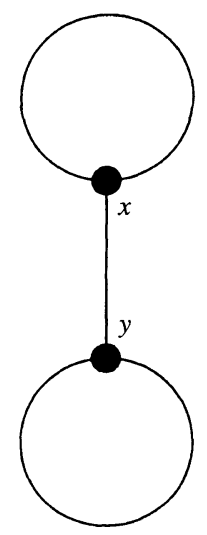

Fig. 3.

However, in fact, it turns out that this moduli space is empty, for generic $f_{i}$. The reason is that there is no periodic orbit of gradient flow except the trivial loop at critical point.

Thus the leading term corresponding to the graph $\Lambda$ is zero. But the correction term may be nonzero, which we are now going to define. Let $p_{i}, q_{i}$ be critical points of $f_{i}$ for $i=1,2$. Assume that $\eta\left(p_{i}\right)=\eta\left(q_{i}\right)+1$. We put

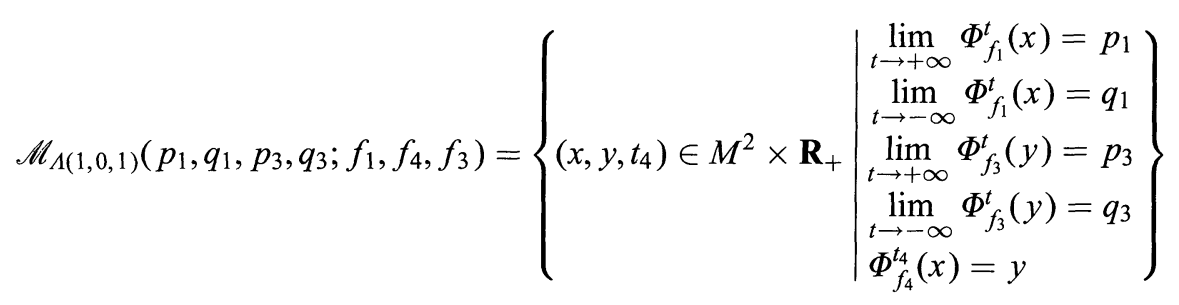

We let $\Lambda(1,0,1)$ be the graph obtained by cutting $\Lambda$ at two points (Fig. 4). $\Lambda(1,0,1)$ consists of 4 edges $e_{1}^{1}, e_{1}^{2}, e_{3}^{1}, e_{3}^{2}, e_{4}$. We define $\chi(I, \varsigma): \varsigma_{p_{1}} \otimes \varsigma_{P_{3}} \rightarrow \varsigma_{q_{1}} \otimes \varsigma_{q_{3}}$ by

$$
\left\langle\chi(I, \varsigma)(u \otimes v), u^{\prime} \otimes v^{\prime}\right\rangle=\sum_{i}\left\langle\left[P_{e_{1}^{1}}(u), P_{e_{1}^{2}}^{-1}(v)\right], \mathbf{e}_{i}\right\rangle\left\langle\left[P_{e_{3}^{1}}\left(u^{\prime}\right), P_{e_{4}}\left(\mathbf{e}_{i}\right)\right], P_{e_{3}^{2}}\left(v^{\prime}\right)\right\rangle .
$$

Here $\mathbf{e}_{i}$ is an orthonormal basis of $\varsigma_{x}$. We then define, for $c_{i} \in \operatorname{End}_{1}\left(C_{*}(M, f, \varsigma)\right)$

$$
\begin{aligned}
Z_{\Lambda}\left(c_{1}, \varnothing, c_{3} ; f_{1}, f_{4}, f_{3}\right)= & \sum_{p_{1}, q_{1}, p_{3}, q_{3} I \in \mathscr{M}_{\Lambda(1,0,1)}} \sum_{\left.p_{1}, q_{1}, p_{2}, q_{2} ; f_{1}, f_{4}, f_{3}\right)} \\
& \pm \operatorname{Tr}\left(\left(c_{1}\left(p_{1}, q_{1}\right) \otimes c_{3}\left(p_{3}, q_{3}\right)\right) \circ \chi(I, \varsigma)\right) .
\end{aligned}
$$

We define $Z_{\Lambda}\left(c_{1}, c_{4}, c_{3} ; f_{1}, f_{4}, f_{3}\right)$ in a similar way. Using them we define

$$
\begin{aligned}
\hat{Z}_{\Lambda}\left(f_{1}, f_{4}, f_{3}\right)= & \frac{1}{8} \sum_{\varepsilon_{i}= \pm 1} Z_{\Lambda(1,0,1)}\left(g_{\varepsilon_{1} f_{1}}, \varnothing, g_{\varepsilon_{3} f_{3}} ; \varepsilon_{1} f_{1}, \varepsilon_{3} f_{3}, \varepsilon_{4} f_{4}\right) \\
& -\frac{1}{8} \sum_{\varepsilon_{l}= \pm 1} Z_{\Lambda(1,1,1)}\left(g_{\varepsilon_{1} f_{1}}, g_{\varepsilon_{4} f_{4}} g_{\varepsilon_{3} f_{3}} ; \varepsilon_{1} f_{1}, \varepsilon_{4} f_{4}, \varepsilon_{3} f_{3}\right) .
\end{aligned}
$$




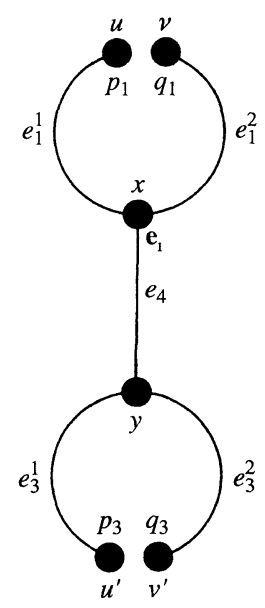

Fig. 4.

We remark here that $C_{*}(M ;-f ; \varsigma)=C_{*}(M ; f ; \varsigma)^{*}$. Hence we have $\operatorname{End}\left(C_{*}(M\right.$; $-f ; \varsigma))=\operatorname{End}\left(C_{*}(M ; f ; \varsigma)^{*}\right)$. We identify $g_{f}$ and $g_{-f}$ by this isomorphism.

We finally define

$$
\begin{aligned}
\hat{Z}_{2}\left(f_{1}, f_{2}, f_{3}, f_{4} ; \varsigma\right)= & \hat{Z}_{\Theta}\left(f_{1}, f_{2}, f_{3} ; \varsigma\right)-\hat{Z}_{\Lambda}\left(f_{1}, f_{2}, f_{4} ; \varsigma\right) \\
& -\hat{Z}_{\Lambda}\left(f_{1}, f_{4}, f_{3} ; \varsigma\right)-\hat{Z}_{\Lambda}\left(f_{4}, f_{2}, f_{3} ; \varsigma\right) .
\end{aligned}
$$

Remark 1.17. We remark that the order of the group of isomorphisms of the $\Theta$ graph is 12 and one of graph $\Lambda$ (graph in Fig. 3 ) is 8 . Hence

$$
\begin{aligned}
& \frac{1}{12}(\text { terms related to } \Theta)-\frac{1}{8}(\text { terms related to } \Lambda) \\
& \quad=\frac{1}{24}(2((\text { terms related to } \Theta)-3(\text { terms related to } \Lambda))
\end{aligned}
$$

is the natural way to sum up contributions from two different graphs. This is the reason why we take $1 / 4$ in place of $1 / 8$ in (1.15). Since we take 3 different choices $\left\{f_{i}, f_{j}\right\} \in\left\{f_{1}, f_{2}, f_{3}\right\}$, we did not put the factor 3 in (1.16).

Now our main result of this paper is as follows.

Main Theorem I. Let $\varsigma, \varsigma^{\prime}$ be the flat Lie algebra bundles with the same Lie algebra $\mathfrak{g}$ as their fibres. Assume $H^{*}(M ; \varsigma)=H^{*}\left(M ; \varsigma^{\prime}\right)=0$. Then the difference, $\hat{Z}_{2}\left(f_{1}, f_{2}, f_{3}, f_{4} ; \varsigma\right)-\hat{Z}_{2}\left(f_{1}, f_{2}, f_{3}, f_{4} ; \varsigma^{\prime}\right)$ is independent of the choice of the Riemannian metric and functions $f_{1}, \ldots, f_{4}$ and combinatorial propagators.

We next assume that $M$ is a homology sphere. Then the Witten complex for the trivial flat bundle is almost acyclic. More precisely we consider the augmentation homomorphism $C_{*}(M, f ; \mathfrak{g}) \rightarrow \mathfrak{g}$ for $*=0,3$. Here we regard $\mathfrak{g}$ as a trivial flat bundle. Let $C_{*}^{\text {red }}(M, f ; \mathfrak{g})$ be its kernel. This complex is acyclic. Therefore we can perform the same construction to obtain $Z_{2}\left(f_{1}, f_{2}, f_{3}, f_{4} ; \mathfrak{g}\right)$. In fact in this case the weight is independent of the element of moduli space and depends only on $\mathfrak{g}$. This 
is because all holonomy is trivial. Let $n_{\mathfrak{g}}$ be this number. $\left(n_{\mathrm{su}(2)}=2^{3}-2=6\right.$ for example.) Then $z_{2}\left(f_{1}, f_{2}, f_{3}, f_{4} ; \mathfrak{g}\right)=n_{\mathfrak{g}} Z_{2}\left(f_{1}, f_{2}, f_{3}, f_{4} ; 1\right)$. We now have:

Main Theorem II. Let $\varsigma$ be the flat Lie algebra bundle with the same Lie algebra $\mathfrak{g}$ as their fibres. Assume $H^{*}(M ; \varsigma)=0$. Then the difference, $\hat{Z}_{2}\left(f_{1}, f_{2}, f_{3}, f_{4} ; \varsigma\right)-$ $n_{\mathrm{g}} \hat{Z}_{2}\left(f_{1}, f_{2}, f_{3}, f_{4} ; 1\right)$ is independent of the choice of the Riemannian metric and functions $f_{1}, \ldots, f_{4}$ and combinatorial propagator.

We remark that our invariant is well defined also for Lie algebra bundle of positive characteristic (if cohomology vanishes) provided the characteristic is not 2 or 3.

Since the correction terms we put look rather complicated at first sight, the author would like to add here some heuristic discussion to show where they come from.

Let us go back to the idea explained in introduction. Namely we consider the set

$$
\mathscr{M}(f)=\left\{(x, y) \in M^{2} \mid \exists t>0 \Phi_{f}^{t}(x)=y\right\} .
$$

The boundary of this set is, roughly speaking, the union of the diagonal and

$$
\mathscr{M}(f, i d)=\bigcup_{p \in \operatorname{Cr}(f)}\left\{(x, y) \in M^{2} \mid \lim _{t \rightarrow \infty} \Phi_{f}^{t}(x)=p=\lim _{t \rightarrow-\infty} \Phi_{f}^{t}(y)\right\} .
$$

(see Lemma 2.4.) We put

$$
\mathscr{M}(f ; p, p)=\left\{(x, y) \in M^{2} \mid \lim _{t \rightarrow \infty} \Phi_{f}^{t}(x)=p=\lim _{t \rightarrow-\infty} \Phi_{f}^{t}(y)\right\} .
$$

As explained in introduction, if we take $\hat{\mathscr{M}}(f)$ such that $\partial \hat{\mathscr{M}}(f)$ is the diagonal, then

$$
\#\left(\hat{\mathscr{M}}\left(f_{1}\right) \cap \hat{\mathscr{M}}\left(f_{2}\right) \cap \hat{\mathscr{M}}\left(f_{3}\right)\right)
$$

is the invariant we want to define. To find such a $\hat{\mathscr{M}}(f)$, we need to find $\mathscr{M}^{\prime}(f)$ such that $\partial \mathscr{M}^{\prime}(f)=\bigcup \mathscr{M}(f ; p, p)$. Our correction term comes from these components.

To explain the reason why the contribution of $\mathscr{M}^{\prime}(f)$ is calculated by the terms we add, let us consider the following situation. We assume that $\operatorname{Cr}(f)=\left\{p_{i}, q_{i}: i=\right.$ $1, \ldots, N\}$, such that $\eta\left(p_{i}\right)=\eta\left(q_{i}\right)+1, \# \overline{\mathscr{M}}\left(p_{i}, q_{i}\right)=1$, and that $\overline{\mathscr{M}}(x, y)$ is empty for any other pair $x, y$ with $\eta(x)=\eta(y)+1$. In fact this assumption is never satisfied because the homology group (with trivial coefficient) of $M$ is always nontrivial. We put this assumption only to simplify the explanation.

We then have that our combinatorial propagator is $\sum_{i}\left[p_{i}\right] \otimes\left[q_{i}\right]^{*}$. We now put

$$
\mathscr{M}^{\prime}(f)=\bigcup_{i}\left\{(x, y) \in M^{2} \mid \lim _{t \rightarrow \infty} \Phi_{f}^{t}(x)=p_{i}, \lim _{t \rightarrow-\infty} \Phi_{f}^{t}(y)=q_{i}\right\} .
$$

We then find that $\partial \mathscr{M}^{\prime}(f)=\bigcup \mathscr{M}\left(f ; p_{i}, p_{i}\right) \cup \bigcup \mathscr{M}\left(f ; q_{i}, q_{i}\right)$ as required. Also we see that

$$
\#\left(\mathscr{M}^{\prime}\left(f_{1}\right) \cap \hat{\mathscr{M}}\left(f_{2}\right) \cap \hat{\mathscr{M}}\left(f_{3}\right)\right)=Z_{\Theta}\left(g_{f_{1}}, \varnothing, \varnothing ; f_{1}, f_{2}, f_{3} ; \text { trivial }\right) .
$$

This explains the origin of our correction terms related to $\Theta$.

To understand why we need to consider the other graph $\Lambda$, we need to consider the transversality at diagonal. One important remark is that for any choice of $f_{i}$ two 
submanifolds $\mathscr{M}\left(f_{1}\right)$ and $\mathscr{M}\left(f_{2}\right)$ are never transversal to each other at diagonal. In fact

$$
\operatorname{dim}\left(\mathscr{M}\left(f_{1}\right) \cap \mathscr{M}\left(f_{2}\right)\right) \geqq \operatorname{dim} \text { of diagonal }=3,
$$

while

$$
\operatorname{dim} \mathscr{M}\left(f_{1}\right)+\operatorname{dim} \mathscr{M}\left(f_{2}\right)-\operatorname{dim} M^{2}=0 .
$$

Therefore we need to study carefully what happens in the neighborhood of diagonal. We remark that this is related to the framing of our manifold $M$, since the framing of $M$ gives a way to perturb the diagonal. This is probably related to the fact that Witten's invariant [W2] depends on the choice of framing. However in this paper we do not use framing directly. Maybe framing is introduced in some sense implicitly when we choose our Morse functions.

We remark that in our situation the transversality at diagonal is related to the compactification of the moduli space $\mathscr{M}\left(f_{1}, f_{2}, f_{3}\right)$ we introduced. As we discuss in next section, the end of this moduli space occurs in two ways. One is the case when $t_{i} \rightarrow \infty$. This end is related to the boundary component $\mathscr{M}(f ; p, p)$ we discussed above. The other case is when $t_{i} \rightarrow 0$. This is related to the transversality we are discussing now.

In Chern-Simons Perturbation theory, two constructions are used to handle the problem related to the transversality of diagonal. One is to consider another graph $\Lambda$ and the other is to cancel anomaly by introducing another term. These two phenomena both have an analogy in our approach.

First we consider also another graph $\Lambda$. These terms are used to cancel the effect of ends such that one of $t_{i} \rightarrow 0$. (see the end of Sect. 3.)

The other is related to the case when all of $t_{i} \rightarrow 0$. This causes some anomaly term also. We kill them by taking the difference of invariant related to two different flat bundles. In place of taking the difference of an invariant determined by different flat bundles there may be an alternative way in the case of homology spheres. We will discuss it informally in Sect. 8 .

\section{Transversality and Compactness}

We begin by proving Lemma 1.2 The idea of the proof is similar to one used in various topological field theories (see for example [FU]) and is described as follows. We first consider the union of moduli spaces $\bigcup_{f_{i} \in C^{\infty}(M)} \mathscr{M}\left(f_{1}, f_{2}, f_{3}\right)$. We then prove that this space is an infinite dimensional manifold. It then follows that $\mathscr{M}\left(f_{1}, f_{2}, f_{3}\right)$ is a manifold for generic $f_{1}, f_{2}, f_{3}$.

We are going to write $\vec{f}$ in place of $\left(f_{1}, f_{2}, f_{3}\right)$ for simplicity. We define $\Phi_{\vec{f}}$ : $M \times \mathbf{R}_{+}^{3} \rightarrow M$ by

$$
\Phi_{\vec{f}}\left(p ; t_{1}, t_{2}, t_{3}\right)=\left(\Phi_{f_{1}}^{t_{1}}(p), \Phi_{f_{2}}^{t_{2}}(p), \Phi_{f_{3}}^{t_{3}}(p)\right) .
$$

Let $\Delta \cong M \subset M^{3}$ be the diagonal. By definition we have $\mathscr{M}_{\Theta}(\vec{f})=\Phi_{\vec{f}}^{-1}(\Delta)$. By moving $\vec{f}$ and also moving the metric we have

$$
\Phi: M \times \mathbf{R}_{+}^{3} \times \operatorname{Met} \times\left(C^{\infty}(M)\right)^{3} \rightarrow M^{3} .
$$

Here Met is the set of all metrics of $M$. 
Lemma 2.1. The map $\Phi$ is transversal to $\Delta$.

Proof. Let $\left(p ; t_{1}, t_{2}, t_{3} ; \vec{f}, g\right)$ be an element of $\Phi^{-1}(\Delta)$. It suffices to show that the differential of $\Phi$ is surjective at this point. We choose disjoint open subsets $U_{i}, i=$ $1,2,3$ of $M$ such that $U_{i} \cap \bigcup_{t \in\left[0, t_{i}\right]} \Phi_{t}^{f_{i}}(p) \neq \varnothing$ and $U_{i} \cap \bigcup_{t \in\left[0, t_{j}\right]} \Phi_{t}^{f_{j}}(p)=\varnothing$ for $i \neq j$. By restricting $\Phi$ to $\left\{\left(p ; t_{1}, t_{2}, t_{3}\right)\right\} \times C_{0}^{\infty}\left(U_{1}\right) \times C_{0}^{\infty}\left(U_{2}\right) \times C_{0}^{\infty}\left(U_{3}\right) \times\{g\}$, we obtain $e v: C_{0}^{\infty}\left(U_{1}\right) \times C_{0}^{\infty}\left(U_{2}\right) \times C_{0}^{\infty}\left(U_{3}\right) \rightarrow M^{3}$. (Here $C_{0}^{\infty}$ is the set of smooth function of compact support.) Namely

$$
\operatorname{ev}\left(h_{1}, h_{2}, h_{3}\right)=\left(\Phi_{f_{1}+h_{1}}^{t_{1}}(p), \Phi_{f_{2}+h_{2}}^{t_{2}}(p), \Phi_{f_{3}+h_{3}}^{t_{3}}(p)\right) .
$$

Let $V_{i} \in T_{\Phi_{f_{i}}^{t_{i}}(p)} M, i=1,2,3$ be arbitrary tangent vectors. By our choice of $U_{i}$ we can find $h_{i}$ such that

$$
\left.\frac{d \Phi_{f_{i}+s h_{i}}^{t_{l}}(p)}{d s}\right|_{s=0}=V_{i} .
$$

It follows that the differential of $\Phi$ at $\left(p ; t_{1}, t_{2}, t_{3}, f_{1}, f_{2}, f_{3}, g\right)$ is surjective. The lemma follows.

We remark that we use the fact $t_{i}>0$ in the proof of the lemma. If we generalize the map $\Phi$ so that it is defined at the point $t_{1}=t_{2}=t_{3}=0$, then the lemma may not hold. (We discuss this point a bit later in this section, when we study the compactness, and we discuss it in more detail in Sect. 6.)

By Lemma 2.1, the set $\Phi^{-1}(\Delta)$ is an infinite dimensional manifold. We consider the projection $\pi: \Phi^{-1}(\Delta) \rightarrow$ Met $\times\left(C^{\infty}(M)\right)^{3}$. Then by a simple counting argument, we obtain the following:

Lemma 2.2. $\pi: \Phi^{-1}(\Delta) \rightarrow$ Met $\times\left(C^{\infty}(M)\right)^{3}$ is a Fredholm map of index $3-$ $\operatorname{dim} M$.

We remark that we do not assume that $\operatorname{dim} M=3$ up to this point. We now assume it. Then by Lemma 2.2 and Sard-Smale theorem [Sm], the space $\mathscr{M}_{\Theta}(\vec{f})=$ $\Phi_{\vec{f}}^{-1}(\Delta)$ is 0 dimensional for generic choice of $\vec{f}$ and metric. We thus proved the first statement of Lemma 1.2 We postpone the discussion about orientation until Sect. 7. We next turn to the proof of the compactness. Before going there we remark that the following one parameter family version of Lemma 1.2 holds. (This is used in a proof of well-definedness in later sections.)

Let $\left(\vec{f}_{(1)}, g_{1}\right),\left(\vec{f}_{(2)}, g_{2}\right)$ be two elements of Met $\times\left(C^{\infty}(M)\right)^{3}$ such that transversality holds for them. We consider a path $L$ joining them. Then Lemma 2.2 and the Sard-Smale theorem again implies that:

Lemma 2.3. For generic $L$ the space, $\pi^{-1}(L)=\bigcup_{(\vec{f}, g) \in L} \mathscr{M}_{\Theta}(\vec{f}, g)$ is one dimensional manifold with boundary $=-\mathscr{M}_{\Theta}\left(\vec{f}_{(1)}, g_{1}\right) \cup \mathscr{M}_{\Theta}\left(\vec{f}_{(2)}, g_{2}\right)$.

We hereafter put $\mathscr{M}_{\Theta}(K)=\bigcup_{(\vec{f}, g) \in K} \mathscr{M}_{\Theta}(\vec{f}, g)$.

Now we begin the discussion of the compactness. First of all we remark that if we fix $\left(t_{1}, t_{2}, t_{3}\right)$ then the space

$$
\left\{\left(p ; t_{1}, t_{2}, t_{3} ; \vec{f}, g\right) \mid\left(p ; t_{1}, t_{2}, t_{3}\right) \in \mathscr{M}_{\Theta}(\vec{f}, g),(\vec{f}, g) \in K\right\}
$$


is compact (if $K$ is compact.) This is a consequence of the fact that our moduli space is the set of solutions of an ordinary differential equation. The analysis one needs to prove this compactness is fairly easy. (In various kinds of topological field theories (such as Yang-Mills theory or pseudo holomorphic curve) one proves a similar compactness theorem using the a priori estimate of partial differential equations.)

Therefore to study the compactness we only need to consider the case when one of $t_{i}$ goes to zero or infinity. This is similar to the bubbling phenomenon which appears in various kinds of topological field theories.

We first study the case when $t_{i} \rightarrow \infty$. In case there is only one Morse function, the study of "bubble" of this kind was used in the proof of the fact that the Witten complex is a complex (namely $\partial \partial=0$.) The argument used there can be applied to our situation without change. (See [Sc] for the detailed account of this argument.) Here we recall the result we need for our purpose. Let $\ell_{i}:\left[0, t_{i}\right] \rightarrow M$ be a sequence of lines such that

$$
\left\{\begin{array}{l}
\frac{d \ell_{i}}{d t}=\operatorname{grad} f \\
t_{i} \rightarrow \infty
\end{array} .\right.
$$

Assume also that $p_{i}=\ell_{i}(0)$ converges to $p \in M$ and $q_{i}=\ell_{i}\left(t_{i}\right)$ converges to $q \in M$. We assume also that $p, q \notin \operatorname{Cr}(f)$. Then we have:

Lemma 2.4. There exists $x_{1}, \ldots, x_{k} \in \mathrm{Cr}(f)$ and gradient lines $m_{j}$ joining $x_{j}$ and $x_{j+1}$. Also there exists a gradient line $m_{p}, m_{q}$ joining $p, x_{k}$ to $x_{1}, q$ respectively, such that the image of $\ell_{i}:\left[0, t_{i}\right] \rightarrow M$ converges to the union of $m_{j}$ and $m_{p}, m_{q}$ with respect to the Hausdorff distance.

We are going to apply this lemma to study the compactification of our moduli space. We first remark that $\operatorname{Cr}\left(f_{i}\right) \cap \operatorname{Cr}\left(f_{j}\right) \neq \varnothing$ at the only codimension 3 subset of $\left(C^{\infty}(M)\right)^{3}$. Since we are considering only at most a 1 dimensional family, we always assume $\operatorname{Cr}\left(f_{i}\right) \cap \operatorname{Cr}\left(f_{j}\right)=\varnothing$ for $i \neq j$. Hereafter we write $\left(C^{\infty}(M)\right)^{3}$ for such a subset for simplicity.

Now we suppose $I_{(i)} \in \mathscr{M}_{\Theta}\left(\vec{f}_{(i)}, g_{i}\right)$, and that $\lim _{i \rightarrow \infty}\left(\vec{f}_{(i)}, g_{i}\right)=(\vec{f}, g)$. We also assume that $I_{(i)}$ is identified to $\left(x_{i}, y_{i} ; t_{1,(i)}, t_{2,(i)}, t_{3,(i)}\right)$ such that $t_{j,(i)}>C$ for some positive $C$ independent of $i$.

We assume that the sequence $I_{(i)}$ diverges. Then at least one of $t_{j,(i)}$ converges to infinity (for some $j=1,2,3$.) Let us assume for simplicity that $t_{1,(i)} \rightarrow \infty$ and the other two are bounded. In fact by a counting argument (similar to those we are going to discuss) we find that in a 0 or 1 dimensional family of $(\vec{f}, g)$ only one of $t_{j,(i)}$ can go to infinity.

Now we apply Lemma 2.4 to the restriction of $I_{(i)}$ to the first edge. The assumption that $x_{i}=I_{(i)}(0)$ converges to $x \in M$ and $y_{i}=I_{(i)}\left(t_{i}\right)$ converges to $y \in M$ is satisfied (after taking a subsequence), since the restriction to $I_{(i)}$ to the other two edges converges. The other assumption that $x, y \notin \operatorname{Cr}\left(f_{1}\right)$ is also satisfied since $x \neq y$. Thus the image of the first edge $e_{1}$ by the map $I_{(i)}$ splits into the union of several gradient lines. Again by a counting argument (which we are soon going to discuss) we may assume that the limit will be the union of 2 gradient lines both of which 
contain a critical point $p$ of $f_{1}$. We then define the following moduli space:

$$
\mathscr{M}_{\Theta(1,0,0)}\left(p, p ; f_{1}, f_{2}, f_{3}\right)=\left\{\begin{array}{l|l}
\left(x, y ; t_{2}, t_{3}\right) \in M^{2} \times \mathbf{R}_{+}^{2} & \begin{array}{l}
\lim _{t \rightarrow+\infty} \Phi_{f_{1}}^{t}(x)=p \\
\lim _{t \rightarrow-\infty} \Phi_{f_{1}}^{t}(y)=p \\
\Phi_{f_{j}}^{t_{j}}(x)=y, \quad j=2,3
\end{array}
\end{array}\right\} .
$$

By moving $\vec{f}$ and the metric in a set $K \subset\left(C^{\infty}(M)\right)^{3} \times$ Met, we define $\mathscr{M}_{\Theta(1,0,0)}(i d, K)$ as follows:

$$
\mathscr{M}_{\Theta(1,0,0)}(i d, K)=\bigcup_{(\vec{f}, g) \in K} \bigcup_{p \in \operatorname{Cr}\left(f_{1}\right)} \mathscr{M}_{\Theta(1,0,0)}\left(p, p ; f_{1}, f_{2}, f_{3}\right) .
$$

We then can prove that $\mathscr{M}_{\Theta(1,0,0)}\left(i d,\left(C^{\infty}(M)\right)^{3} \times\right.$ Met $)$ is an infinite dimensional manifold in a way similar to the proof of Lemma 2.1. We find that the projection $\mathscr{M}_{\Theta(1,0,0)}\left(i d,\left(C^{\infty}(M)\right)^{3} \times\right.$ Met $) \rightarrow\left(C^{\infty}(M)\right)^{3} \times$ Met is a Fredholm map of index -1 . The calculation of the index is done as follows. In the definition of $\mathscr{M}_{\Theta(1,0,0)}(p, p ; \vec{f})$ there are 2 variables moving on $M$ and two positive numbers. For the equation, the first equation puts $\eta(p)$ conditions. The second equation puts $3-\eta(p)$ equation. The two equations in the third line puts 3 conditions each. In total the index is $3 \times 2+2-\eta(p)-(3-\eta(p))-6=-1$.

Thus using the Sard-Smale theorem again, we find that, in the case when we fix a generic $\vec{f}$ there is no such an end. And in case $\vec{f}$ moves in a generic one dimensional set there are finitely many points like that.

Next we turn to the case when one of $t_{j,(i)}$, say $t_{1,(i)}$ goes to zero. Namely we assume that $\left(x_{i}, y_{i} ; t_{1,(i)}, t_{2,(i)}, t_{3,(i)}=I_{(i)} \in \mathscr{M}_{\Theta}\left(\vec{f}_{(i)}, g_{i}\right), \quad \lim _{i \rightarrow \infty} t_{1,(i)}=0\right.$, $\lim _{i \rightarrow \infty}\left(\vec{f}_{(i)}, g_{i}\right)=(\vec{f}, g)$. By assumption $\lim _{i \rightarrow \infty} t_{1,(i)}=0$ we have (by taking a subsequence if necessary) that $\lim _{i \rightarrow \infty} x_{i}=\lim _{i \rightarrow \infty} y_{i}=x$. Since $\operatorname{Cr}\left(f_{2}\right) \cap \operatorname{Cr}\left(f_{3}\right)=$ $\varnothing$, we may assume without loss of generality that $x \notin \operatorname{Cr}\left(f_{3}\right)$. Then there are two cases.

Case 1. $x \notin \operatorname{Cr}\left(f_{2}\right)$. In this case we have $\lim _{i \rightarrow \infty} t_{2,(i)}=\lim _{i \rightarrow \infty} t_{3,(i)}=0$. We can then use the fact $\Phi_{f_{j},(i)}^{t_{j},(i)}\left(x_{i}\right)=y_{i}$ to show that three vectors $\operatorname{grad} f_{j}(x), j=1,2,3$ are parallel to each other.

Case 2. $x \in \operatorname{Cr}\left(f_{2}\right)$. In this case we find $\operatorname{grad} f_{j}(x), j=1,2$ are parallel. Since $\operatorname{grad} f_{2}(x)=0$, we may say that three vectors $\operatorname{grad} f_{j}(x), j=1,2,3$ are parallel also in this case.

Here we say that $\operatorname{grad} f_{i}(x)$ and $\operatorname{grad} f_{j}(x)$ are parallel if there exists a nonnegative number $r_{i}, r_{j}$ such that $r_{i} \operatorname{grad} f_{i}(x)=r_{j} \operatorname{grad} f_{j}(x)$ and $\left(r_{i}, r_{j}\right) \neq(0,0)$. (This notation is a bit different from usual one, since we avoid, for example $\operatorname{grad} f_{i}(x)=-\operatorname{grad} f_{j}(x)$.)

Now we put

$$
R(\vec{f})=\left\{x \in M \mid \operatorname{grad} f_{j}(x) j=1,2,3 \text { are parallel to each other }\right\} .
$$

For $K \subseteq\left(C^{\infty}(M)\right)^{3} \times$ Met we put

$$
R(K)=\{((\vec{f}, g), x) \mid(\vec{f}, g) \in K, x \in R(\vec{f})\}
$$


Lemma 2.5. $R\left(\left(C^{\infty}(M)\right)^{3} \times\right.$ Met $)$ is an infinite dimensional manifold. And the map $\pi: R\left(\left(C^{\infty}(M)\right)^{3} \times \mathrm{Met}\right) \rightarrow\left(C^{\infty}(M)\right)^{3} \times$ Met is a Fredholm map of index -1 .

The proof of the first statement is a standard transversality argument and the proof of the second statement is a simple dimension counting.

Lemma 2.5 and Sard-Smale theorem imply that for generic $(\vec{f}, g)$, the set $R(\vec{f})$ is empty. Thus we have proved the following:

Lemma 2.6. For generic $(\vec{f}, g)$ the space $\mathscr{M}_{\Theta}(\vec{f}, g)$ is compact.

This completes the proof of Lemma 1.2 except orientation.

We turn to study $\mathscr{M}_{\Theta}(L)$ for the generic one dimensional space $L \subseteq\left(C^{\infty}(M)\right)^{3} \times$ Met. We already discussed most of the phenomena which can occur. One is described by the space $\mathscr{M}_{\Theta(1,0,0)}(L) \cup \mathscr{M}_{\Theta(0,1,0)}(L) \cup \mathscr{M}_{\Theta(0,0,1)}(L)$ (which corresponds to the case when $t_{j} \rightarrow \infty$ ) the other is described by the set $R(L)$. For each element of $\mathscr{M}_{\Theta(1,0,0)}(L) \cup \mathscr{M}_{\Theta(0,1,0)}(L) \cup \mathscr{M}_{\Theta(0,0,1)}(L)$ we can construct an end of $\mathscr{M}_{\Theta}(L)$ homeomorphic to $[0, \infty)$. This fact is based on a converse to Lemma 2.4 which was also proved and used in the construction of the Witten complex.

We can prove also that, for each element of $R(L)$, there exists an end of $\mathscr{M}_{\Theta}(L)$ which is homeomorphic to $[0, \infty)$, if $L$ is generic. The proof of this fact is more delicate and will be given in Sect. 6. We observe that elements of $\mathscr{M}_{\Theta}(L)$ which are in an end corresponding to an element of $R(L)$ are of small diameter. Hence they are contractible. This fact is important since it follows that the weight associate to such an element is independent of the flat bundle $\varsigma$. Therefore, since we consider the difference of invariants obtained from 2 flat bundles, the contribution from this kind of end cancels automatically. We summarize our result as follows.

Lemma 2.7. Let $L \subseteq\left(C^{\infty}(M)\right)^{3} \times$ Met be a generic arc joining two generic elements of $\left(C^{\infty}(M)\right)^{3} \times$ Met. Then there exists a one dimensional compact manifold $\mathscr{C} \mathscr{M}_{\Theta}(L)$ with boundary such that $\mathscr{M}_{\Theta}(L)$ is its interior and $\mathscr{C} \mathscr{M}_{\Theta, L}(\vec{f})-$ $\mathscr{M}_{\Theta, L}(\vec{f})=\mathscr{M}_{\Theta(1,0,0)}(L) \cup \mathscr{M}_{\Theta(0,1,0)}(L) \cup \mathscr{M}_{\Theta(0,0,1)}(L) \cup R(L)$.

In later sections, we use this and similar lemmas to prove the well-definedness of our invariant.

\section{Independence of Combinatorial Propagator}

To prove our main theorem (independence of invariant of various choices) we first consider, in this section, the independence of the combinatorial propagator. We assume that we have two choices $g_{f_{1}}, g_{f_{1}}^{\prime}$ of the combinatorial propagator for the first Morse function $f_{1}$ and assume that other combinatorial propagators $g_{f_{i}}, i=2,3,4$ are the same. And we prove that the resulting invariants are the same.

Since the Witten complex of local coefficient are acyclic by our assumption, there is $h \in \operatorname{End}_{2}\left(C_{*}\left(M ; f_{1} ; \varsigma\right)\right.$ such that $\partial h=g_{f_{1}}-g_{f_{1}}^{\prime}$. We put

$$
h=\sum_{\eta\left(p_{1}\right)=\eta\left(q_{1}\right)+2} h\left(p_{1}, q_{2}\right)\left[p_{1}\right] \otimes\left[q_{1}\right]^{*} .
$$

We are going to study the moduli space $\mathscr{M}_{\Theta(1,0,0)}\left(p_{1}, q_{1}\right)$ in the case $\eta\left(p_{1}\right)=$ $\eta\left(q_{1}\right)+2$. (Since we fix $f_{i}$ here we omit them in the above notation.) In this case 
the moduli space is one dimensional. (One needs transversality to prove it. It can be proved in the same way as in the last section.) We need to know the ideal boundary of this moduli space $\mathscr{M}_{\Theta(1,0,0)}\left(p_{1}, q_{1}\right)$.

Lemma 3.1. $\mathscr{M}_{\Theta(1,0,0)}\left(p_{1}, q_{1}\right)$ is an interior of a compact oriented one dimensional manifold $\mathscr{C} \mathscr{M}_{\Theta(1,0,0)}\left(p_{1}, q_{1}\right)$ such that

$$
\begin{aligned}
\partial \mathscr{C} \mathscr{M}_{\Theta(1,0,0)}\left(p_{1}, q_{1}\right)= & \bigcup_{\eta\left(p_{1}^{\prime}\right)=\eta\left(p_{1}\right)-1} \overline{\mathscr{M}}\left(p_{1}, p_{1}^{\prime}\right) \times \mathscr{M}_{\Theta(1,0,0)}\left(p_{1}^{\prime}, q_{1}\right) \\
& \cup \bigcup_{\eta\left(q_{1}^{\prime}\right)=\eta\left(q_{1}\right)+1} \mathscr{M}_{\Theta(1,0,0)}\left(p_{1}, q_{1}^{\prime}\right) \times \overline{\mathscr{M}}\left(q_{1}^{\prime}, q_{1}\right) \\
& \cup \bigcup_{p_{2} \in \operatorname{Cr}\left(f_{2}\right)} \mathscr{M}_{\Theta(1,1,0)}\left(p_{1}, q_{1} ; p_{2}, p_{2}\right) \\
& \cup \bigcup_{p_{3} \in \operatorname{Cr}\left(f_{3}\right)} \mathscr{M}_{\Theta(1,0,1)}\left(p_{1}, q_{1} ; p_{3}, p_{3}\right) \cup \mathscr{S}_{\Theta(1,0,0)}\left(p_{1}, q_{1}\right) .
\end{aligned}
$$

Let us explain the notations above. We define

$$
\mathscr{M}_{\Theta(1,0,1)}\left(p_{1}, q_{1} ; p_{3}, p_{3}\right)=\left\{\begin{array}{l|l}
\left(x, y ; t_{2}\right) \in M^{2} \times \mathbf{R}_{+} & \begin{array}{l}
\lim _{t \rightarrow \infty} \Phi_{f_{1}}^{t}(x)=p_{1}, \\
\lim _{t \rightarrow-\infty} \Phi_{f_{1}}^{t}(y)=q_{1} \\
\lim _{t \rightarrow \infty} \Phi_{f_{3}}^{t}(x)=p_{3}, \\
\lim _{t \rightarrow-\infty} \Phi_{f_{1}}^{t}(x)=q_{3} \\
\Phi_{f_{2}}^{t_{2}}(x)=y
\end{array}
\end{array}\right\}
$$

and

$$
\mathscr{S}_{\Theta(1,0,0)}\left(\vec{f}, g ; p_{1}, q_{1}\right)=\left\{\begin{array}{l|l}
x \in M & \begin{array}{l}
\lim _{t \rightarrow-\infty} \Phi_{f_{1}}^{t}(x)=p_{1} \\
\lim _{t \rightarrow+\infty} \Phi_{f_{1}}^{t}(x)=q_{1} \\
\operatorname{grad} f_{2}(x) \text { is parallel to grad } f_{3}(x)
\end{array}
\end{array}\right\} .
$$

Other notations are defined in a similar way.
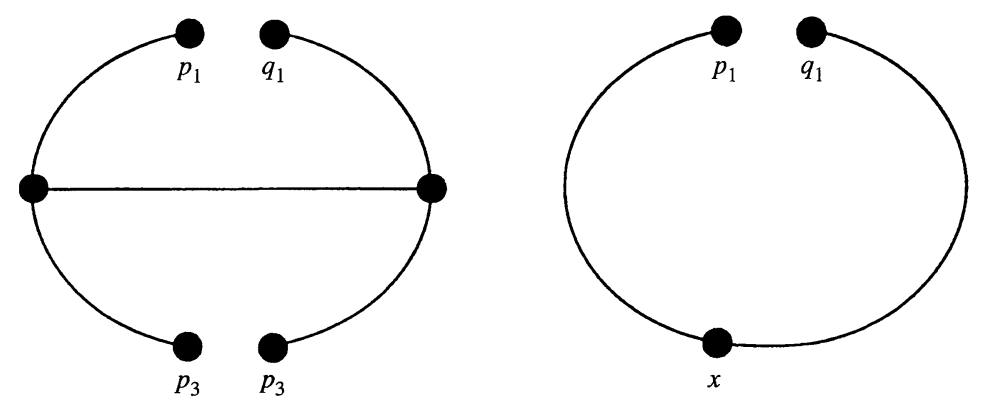

Fig. 5. 
Proof. The proof of Lemma 3.1 goes almost in the same way as Sect. 2.

Let $\left(x_{i}, y_{i}, t_{2}^{(i)}, t_{3}^{(i)}\right) \in \mathscr{M}_{\Theta(1,0,0)}\left(p_{1}, q_{1}\right)$ be a divergence sequence. Then by taking a subsequence if necessary, we may assume that one of the following happens.

(1) $t_{2}^{(i)} \rightarrow \infty$,

(2) $t_{3}^{(i)} \rightarrow \infty$,

(3) $x_{i}$ converges to a point in the boundary of the unstable manifold of $p_{1}$,

(4) $y_{i}$ converges to a point in the boundary of the stable manifold of $q_{1}$,

(5) $t_{2}^{(i)} \rightarrow 0$ or $t_{3}^{(i)} \rightarrow 0$.

It is straightforward to see that cases $(1) \sim(5)$ correspond to the $1 \sim 5^{\text {th }}$ term of the right-hand side of Lemma 3.1. It is easy to see that, for each element in $1 \sim 4^{\text {th }}$ term of the right-hand side of Lemma 3.1, there exists an end of $\mathscr{M}_{\Theta(1,0,0)}\left(p_{1}, q_{1}\right)$ corresponding to it. We postpone to Sect. 6 , the proof of the fact that there is also an end of $\mathscr{M}_{\Theta(1,0,0)}\left(p_{1}, q_{1}\right)$ corresponding to each element of $\mathscr{S}_{\Theta(1,0,0)}\left(\vec{f}, g ; p_{1}, q_{1}\right)$. We here prove that $\mathscr{S}_{\Theta(1,0,0)}\left(\vec{f}, g ; p_{1}, q_{1}\right)$ is zero dimensional.

We prove this fact again by using transversality. We put

$$
\mathscr{S}_{\Theta(1,0,0), 2}=\bigcup_{(\vec{f}, g) \in\left(C^{\infty}(M)\right)^{3} \times \operatorname{Met}} \bigcup_{\substack{p_{1}, q_{1} \in \operatorname{Cr}\left(f_{1}\right) \\ \eta\left(p_{1}\right)=\eta\left(q_{1}\right)+2}} \mathscr{S}_{\Theta(1,0,0)}\left(\vec{f}, g ; p_{1}, q_{1}\right) .
$$

We then have:

Lemma 3.2. The space $\mathscr{S}_{\Theta(1,0,0), 2}$ is an (infinite dimensional) manifold and the projection: $\mathscr{S}_{\Theta(1,0,0), 2} \rightarrow\left(C^{\infty}(M)\right)^{3} \times$ Met is a Fredholm map of index 0 .

Lemma 3.2 is a consequence of the usual dimension counting and transversality.

It follows immediately from the lemma that $\mathscr{S}_{\Theta(1,0,0)}\left(\vec{f}, g ; p_{1}, q_{1}\right)$ is zero dimensional for $\eta\left(p_{1}\right)=\eta\left(q_{1}\right)+2$. The proof of Lemma 3.1 is now complete.

In order to deduce identities from Lemma 3.1, we are going to define a number $Z_{\Theta(1,1,0)}\left(c_{1}, c_{2}, \varnothing\right)$ for $c_{1} \in \operatorname{End}_{2}\left(C_{*}\left(M ; f_{1} ; \varsigma\right)\right), c_{2} \in \operatorname{End}_{1}\left(C_{*}\left(M ; f_{2} ; \varsigma\right)\right)$. Let us write

$$
c_{j}=\sum c_{j}\left(p_{j}, q_{j}\right)\left[p_{j}\right] \otimes\left[q_{j}\right]^{*}, \quad j=1,2 .
$$

An element of the moduli space $\mathscr{M}_{\Theta(1,1,0)}\left(p_{1}, q_{1} ; p_{2}, q_{2}\right)$ is regarded as a map $I: \Theta(1,1,0) \rightarrow M$. Here $\Theta(1,1,0)$ is a graph obtained by cutting the $\Theta$ graph at two points, one on the first edge and the other on the second edge. We define the weight $\chi(I): \eta_{p_{1}} \otimes \eta_{p_{2}} \rightarrow \eta_{q_{1}} \otimes \eta_{q_{2}}$ as we did in Sect. 1. We now put

$$
Z_{\Theta(1,1,0)}\left(c_{1}, c_{2}, \varnothing\right)=\sum_{p_{1}, q_{1}, p_{2}, q_{2}} \sum_{I \in M_{\Theta}\left(p_{1}, q_{1}, p_{2}, q_{2}\right)} \pm \operatorname{Tr}\left(\chi(I) \circ\left(c_{1}\left(p_{1}, q_{1}\right) \otimes c_{2}\left(p_{2}, q_{2}\right)\right)\right) .
$$

To study the fifth term we define

$$
Z S_{\Theta(1,0,0)}\left(c_{1}, \varnothing, \varnothing\right)=\sum_{p_{1}, q_{1}} \sum_{I \in \mathscr{S}_{\Theta(1,0,0)}\left(p_{1}, q_{1}\right)} \pm \operatorname{Tr}\left(\chi(I) \circ\left(c_{1}\left(p_{1}, q_{1}\right)\right)\right.
$$

Here $\chi(I): \eta_{p} \rightarrow \eta_{q}$ is a parallel transport along the gradient line of $f_{1}$ containing $x$. (Here we regard $x \in M$.)

Now Lemma 3.1 implies: 


\section{Lemma 3.3.}

$$
\begin{aligned}
Z_{\Theta(1,0,0)}\left(g_{f_{1}}^{\prime}, \varnothing, \varnothing\right)= & Z_{\Theta(1,0,0)}\left(g_{f_{1}}, \varnothing, \varnothing\right)-Z_{\Theta(1,1,0)}(h, i d, \varnothing) \\
& -Z_{\Theta(1,0,1)}(h, \varnothing, i d)-Z S_{\Theta(1,0,0)}(h, \varnothing, \varnothing) .
\end{aligned}
$$

We can also prove the following formula:

$$
\begin{aligned}
Z_{\Theta(1,1,1)}\left(g_{f_{1}}^{\prime}, g_{f_{2}}, g_{f_{3}}\right)= & Z_{\Theta(1,1,1)}\left(g_{f_{1}}, g_{f_{2}}, g_{f_{3}}\right)-Z_{\Theta(1,1,1)}\left(h, \partial g_{f_{2}}, g_{f_{3}}\right) \\
& -Z_{\Theta(1,1,1)}\left(h, g_{f_{2}}, \partial g_{f_{3}}\right) .
\end{aligned}
$$

Equation (3.4) follows from the following description of the boundary of moduli spaces:

$$
\begin{aligned}
\partial \mathscr{C} \mathscr{M}_{\Theta(1,1,0)}\left(p_{1}, q_{1} ; p_{2}, q_{2} ; p_{3}, q_{3}\right) \\
=\bigcup_{\eta\left(p_{1}^{\prime}\right)=\eta\left(p_{1}\right)-1} \overline{\mathscr{M}}\left(p_{1}, p_{1}^{\prime}\right) \times \mathscr{M}_{\Theta(1,1,1)}\left(p_{1}^{\prime}, q_{1} ; p_{2}, q_{2} ; p_{3}, q_{3}\right) \\
\quad \cup \bigcup_{\eta\left(q_{1}^{\prime}\right)=\eta\left(q_{1}\right)+1} \overline{\mathscr{M}}\left(q_{1}^{\prime}, q_{1}\right) \times \mathscr{M}_{\Theta(1,1,1)}\left(p_{1}, q_{1}^{\prime} ; p_{2}, q_{2} ; p_{3}, q_{3}\right) \\
\quad \cup \bigcup_{\eta\left(p_{2}^{\prime}\right)=\eta\left(p_{2}\right)-1} \overline{\mathscr{M}}\left(p_{2}, p_{2}^{\prime}\right) \times \mathscr{M}_{\Theta(1,1,1)}\left(p_{1}, q_{1} ; p_{2}^{\prime}, q_{2} ; p_{3}, q_{3}\right) \\
\quad \cup \bigcup_{\eta\left(q_{2}^{\prime}\right)=\eta\left(q_{2}\right)+1} \overline{\mathscr{M}}\left(q_{2}^{\prime}, q_{2}\right) \times \mathscr{M}_{\Theta(1,1,1)}\left(p_{1}, q_{1} ; p_{2}, q_{2}^{\prime} ; p_{3}, q_{3}\right) \\
\quad \cup \bigcup_{\eta\left(p_{3}^{\prime}\right)=\eta\left(p_{3}\right)-1} \overline{\mathscr{M}}\left(p_{3}, p_{3}^{\prime}\right) \times \mathscr{M}_{\Theta(1,1,1)}\left(p_{1}, q_{1} ; p_{2}, q_{2} ; p_{3}^{\prime}, q_{3}\right) \\
\quad \cup \underset{\eta\left(q_{3}^{\prime}\right)=\eta\left(q_{3}\right)+1}{\bigcup} \overline{\mathscr{M}}\left(q_{3}^{\prime}, q_{3}\right) \times \mathscr{M}_{\Theta(1,1,1)}\left(p_{1}, q_{1} ; p_{2}, q_{2} ; p_{3}, q_{3}^{\prime}\right) .
\end{aligned}
$$

We can prove this equality in a way similar to Lemma 3.1. We remark that we do not need the term $\mathscr{S}_{\Theta(1,0,0)}\left(p_{1}, q_{1}\right)$.

To consider the term $Z_{\Theta(1,1,0)}\left(g_{f_{1}}, g_{f_{2}}, \varnothing\right)$ and like that, we need to study another kind of degeneration. To describe it we use the following moduli space:

$$
\mathscr{M}_{\Theta(1,1, X)}\left(p_{1}, q_{1} ; p_{2}, q_{2}\right)=\left\{\begin{array}{l|l}
x \in M & \begin{array}{l}
\lim _{t \rightarrow+\infty} \Phi_{f_{1}}^{t}(x)=p_{1}, \\
\lim _{t \rightarrow-\infty} \Phi_{f_{1}}^{t}(x)=q_{1} \\
\lim _{t \rightarrow+\infty} \Phi_{f_{2}}^{t}(x)=p_{2}, \\
\lim _{t \rightarrow-\infty} \Phi_{f_{2}}^{t}(x)=q_{2}
\end{array}
\end{array}\right\} .
$$

(This moduli space is the case when $t_{3}=0$.) We can assume that unstable and stable manifolds of $f_{1}, f_{2}, f_{3}$ are transversal to each other. So in case $\eta\left(p_{1}\right)=$ $\eta\left(q_{1}\right)+2, \eta\left(p_{2}\right)=\eta\left(q_{2}\right)+1$, the space $\mathscr{M}_{\Theta(1,1, X)}\left(p_{1}, q_{1} ; p_{2}, q_{2}\right)$ is 0 -dimensional and compact. Using this space we can describe the boundary of the moduli space 
$\mathscr{M}_{\Theta(1,1,0)}\left(p_{1}, q_{1} ; p_{2}, q_{2}\right)$ as follows:

$$
\begin{aligned}
& \partial \mathscr{C} \mathscr{M}_{\Theta(1,1,0)}\left(p_{1}, q_{1} ; p_{2}, q_{2}\right) \\
& =\bigcup_{\eta\left(p_{1}^{\prime}\right)=\eta\left(p_{1}\right)-1} \overline{\mathscr{M}}\left(p_{1}, p_{1}^{\prime}\right) \times \mathscr{M}_{\Theta(1,1,0)}\left(p_{1}^{\prime}, q_{1} ; p_{2}, q_{2}\right) \\
& \quad \cup \bigcup_{\eta\left(q_{1}^{\prime}\right)=\eta\left(q_{1}\right)+1} \overline{\mathscr{M}}\left(q_{1}^{\prime}, q_{1}\right) \times \mathscr{M}_{\Theta(1,1,0)}\left(p_{1}, q_{1}^{\prime} ; p_{2}, q_{2}\right) \\
& \quad \cup \bigcup_{\eta\left(p_{2}^{\prime}\right)=\eta\left(p_{2}\right)-1} \overline{\mathscr{M}}\left(p_{2}, p_{2}^{\prime}\right) \times \mathscr{M}_{\Theta(1,1,0)}\left(p_{1}, q_{1} ; p_{2}^{\prime}, q_{2}\right) . \\
& \quad \cup \bigcup_{\eta\left(q_{2}^{\prime}\right)=\eta\left(q_{2}\right)+1} \overline{\mathscr{M}}\left(q_{2}^{\prime}, q_{2}\right) \times \mathscr{M}_{\Theta(1,1,0)}\left(p_{1}, q_{1} ; p_{2}, q_{2}^{\prime}\right) \\
& \quad \cup \underset{p_{3} \in \operatorname{Cr}\left(f_{3}\right)}{\bigcup \mathscr{M}_{\Theta(1,1,1)}\left(p_{1}, q_{1} ; p_{2}, q_{2} ; p_{3}, p_{3}\right)} \\
& \quad \cup \mathscr{M}_{\Theta(1,1, X)}\left(p_{1}, q_{1} ; p_{2}, q_{2}\right) .
\end{aligned}
$$

We define the number $Z_{\Theta(1,1, X)}\left(h, g_{f_{2}}, \varnothing\right)$ using the moduli space $\mathscr{M}_{\Theta(1,1, X)}\left(p_{1}\right.$, $\left.q_{1} ; p_{2}, q_{2}\right)$ in a similar way. Then (3.5) implies the following:

$$
\begin{aligned}
Z_{\Theta(1,1,0)}\left(g_{f_{1}}^{\prime}, g_{f_{2}}, \varnothing\right)= & Z_{\Theta(1,0,0)}\left(g_{f_{1}}, g_{f_{2}}, \varnothing\right)+Z_{\Theta(1,1,0)}\left(h, \partial g_{f_{2}}, \varnothing\right) \\
& +Z_{\Theta(1,1,0)}\left(h, g_{f_{2}}, i d\right)+Z_{\Theta(1,1, X)}\left(h, g_{f_{2}}, \varnothing\right) .
\end{aligned}
$$

Now we use the fact that $\partial g_{f_{j}}=i d$. Then Lemma 3.3, (3.4), (3.6) imply:

\section{Lemma 3.7.}

$$
\begin{aligned}
\hat{\bar{Z}}_{\Theta}^{\prime}(\vec{f}, g)-\hat{\bar{Z}}_{\Theta}(\vec{f}, g)= & Z_{\Theta(1,1, X)}\left(h, g_{f_{2}}, \varnothing\right)+Z_{\Theta(1, X, 1)}\left(h, \varnothing, g_{f_{3}}\right) \\
& +Z S_{\Theta(1,1,0)}(h, \varnothing, \varnothing) .
\end{aligned}
$$

We next sum them up for signs $\varepsilon_{j}$ of $f_{j}$. We then remark the following:

\section{Lemma 3.8.}

$Z S_{\Theta(1,0,0)}\left(f_{1}, f_{2}, f_{3} ; h_{f_{1}}, \varnothing, \varnothing\right)=-Z S_{\Theta(1,0,0)}\left(-f_{1}, f_{2}, f_{3} ; h_{-f_{1}}, \varnothing, \varnothing\right)$.

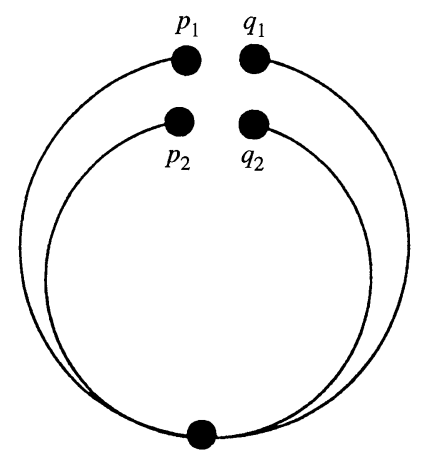

Fig. 6. 
Proof. We first remark that the equality between moduli spaces $\mathscr{M}_{S \Theta}\left(p_{1}, q_{1} ; f_{1}, f_{2}\right.$, $\left.f_{3}\right)=\mathscr{M}_{S \Theta}\left(q_{1}, p_{1} ;-f_{1}, f_{2}, f_{3}\right)$. The orientation is opposite as we will see in Sect. 7. We remark also that the isomorphism $\operatorname{End}\left(C_{*}(M ; f ; \varsigma)\right)=\operatorname{End}\left(C_{*}(M ;-f ; \varsigma)\right)$ is obtained by $c[p] \otimes[q]^{*} \mapsto c[q] \otimes[p]^{*}$. The lemma then follows immediately from the definition.

Using Lemmas 3.7 and 3.8 we have

\section{Lemma 3.9.}

$$
\begin{aligned}
4 \hat{Z}_{\Theta}^{\prime}(\vec{f}, g)-4 \hat{Z}_{\Theta}(\vec{f}, g)= & \sum_{\varepsilon_{1}, \varepsilon_{2}, \varepsilon_{3}= \pm 1} Z_{\Theta(1,1, X)}\left(\varepsilon_{1} f_{1}, \varepsilon_{2} f_{2}, \varepsilon_{3} f_{3} ; h_{\varepsilon_{1} f_{1}}, g_{\varepsilon_{2} f_{2}}, \varnothing\right) \\
& +\sum_{\varepsilon_{1}, \varepsilon_{2}, \varepsilon_{3}= \pm 1} Z_{\Theta(1, X, 1)}\left(\varepsilon_{1} f_{1}, \varepsilon_{2} f_{2}, \varepsilon_{3} f_{3} ; h_{\varepsilon_{1} f_{1}}, \varnothing, g_{\varepsilon_{3} f_{3}}\right) .
\end{aligned}
$$

The proof that this last term cancels with the corresponding term related to the other graph $\Lambda$ uses the Jacobi identity and is similar to an argument in ChernSimons Perturbation theory. We are going to explain it here.

We perform the same construction using the other graph $\Lambda$ and obtain the equality

$$
\begin{aligned}
8\left(\hat{Z}_{\Lambda}^{\prime}-\hat{Z}_{\Lambda}\right)= & \sum_{\varepsilon_{1}, \varepsilon_{2}, \varepsilon_{4}= \pm 1} Z_{\Lambda(1,1, X)}\left(h_{\varepsilon_{1} f_{1}}, g_{\varepsilon_{2} f_{2}}, \varnothing\right)\left(\varepsilon_{1} f_{1}, \varepsilon_{2} f_{2}, \varepsilon_{4} f_{4}\right) \\
& +\sum_{\varepsilon_{1}, \varepsilon_{2}, \varepsilon_{4}= \pm 1} Z_{\Lambda(1, X, 1)}\left(h_{\varepsilon_{1} f_{1}}, \varnothing, g_{\varepsilon_{3} f_{3}}\right)\left(\varepsilon_{1} f_{1}, \varepsilon_{4} f_{4}, \varepsilon_{3} f_{3}\right) .
\end{aligned}
$$

Here the number $Z_{\Lambda(1,1, X)}\left(h_{\varepsilon_{1} f_{1}}, g_{\varepsilon_{2} f_{2}}, \varnothing\right)\left(\varepsilon_{1} f_{1}, \varepsilon_{2} f_{2}, \varepsilon_{4} f_{4}\right)$ is defined by using moduli space $\mathscr{M}_{\Lambda(1,1, X)}\left(p_{1}, q_{1}, p_{2}, q_{2}\right)\left(\varepsilon_{1} f_{1}, \varepsilon_{2} f_{2}, \varepsilon_{4} f_{4}\right)$, which is a component of the boundary of $\mathscr{M}_{\Lambda(1,1,0)}\left(p_{1}, q_{1}, p_{2}, q_{2}\right)\left(\varepsilon_{1} f_{1}, \varepsilon_{2} f_{2}, \varepsilon_{4} f_{4}\right)$ corresponding to $t_{4}=0$.

We next remark the following obvious facts:

$$
\begin{aligned}
\mathscr{M}_{\Theta(1,1, X)}\left(p_{1}, q_{1}, p_{2}, q_{2}\right)\left(f_{1}, f_{2}, \pm f_{3}\right) & =\mathscr{M}_{\Theta(1,1, X)}\left(q_{1}, p_{1}, p_{2}, q_{2}\right)\left(-f_{1}, f_{2}, \pm f_{3}\right) \\
& =\mathscr{M}_{\Theta(1,1, X)}\left(p_{1}, q_{1}, q_{2}, p_{2}\right)\left(f_{1},-f_{2}, \pm f_{3}\right) \\
& =\mathscr{M}_{\Theta(1,1, X)}\left(q_{1}, p_{1}, q_{2}, p_{2}\right)\left(-f_{1},-f_{2}, \pm f_{3}\right) \\
& =\mathscr{M}_{\Lambda(1,1, X)}\left(p_{1}, q_{1}, p_{2}, q_{2}\right)\left(f_{1}, f_{2}, \pm f_{4}\right) \\
& =\mathscr{M}_{\Lambda(1,1, X)}\left(p_{1}, q_{1}, q_{2}, p_{2}\right)\left(f_{1},-f_{2}, \pm f_{4}\right) \\
& =\mathscr{M}_{\Lambda(1,1, X)}\left(q_{1}, p_{1}, p_{2}, q_{2}\right)\left(-f_{1}, f_{2}, \pm f_{4}\right) \\
& =\mathscr{M}_{\Lambda(1,1, X)}\left(q_{1}, p_{1}, q_{2}, p_{2}\right)\left(-f_{1},-f_{2}, \pm f_{4}\right),
\end{aligned}
$$

and also a similar formula with $p_{2}, q_{2}, f_{2}$ replaced by $p_{3}, q_{3}, f_{3}$ holds. To show the cancellation we need to discuss the weights.

The moduli space in Formula (3.10) is also described as the set $U\left(p_{1}\right) \cap S\left(q_{1}\right) \cap$ $U\left(p_{2}\right) \cap S\left(q_{2}{ }^{*}\right)$. Here $U(\cdot)$ is the unstable manifold and $S(\cdot)$ is the stable manifold. Hence for each point $x$ of $U\left(p_{1}\right) \cap S\left(q_{1}\right) \cap U\left(p_{2}\right) \cap S\left(q_{2}\right)$, there are 4 gradient lines joining it to $p_{1}, q_{1}, p_{2}, q_{2}$ respectively. Let $P_{p_{l}, x}: \varsigma_{p_{i}} \rightarrow \varsigma_{x}, P_{q_{l}, x}: \varsigma_{q_{i}} \rightarrow \varsigma_{x}$ be the parallel translation along these paths. The weight of $x$ gives a map $\varsigma_{p_{1}} \otimes \varsigma_{q_{1}} \rightarrow$ $\varsigma_{p_{2}} \otimes \varsigma_{q_{2}}$. But the weight does depend on the moduli space whose element $x$ is 
regarded as. Let us regard $x \in \mathscr{M}_{\Theta(1,1, X)}\left(p_{1}, q_{1}, p_{2}, q_{2} ; f_{1}, f_{2}, \pm f_{3}\right)$. In other words, $x$ is regarded as a map from the $\Theta$-graph such that the third edge is mapped to the point $x$. Therefore, for $u \in \varsigma_{p_{1}}, v \in \varsigma_{p_{2}}, u^{\prime} \in \varsigma_{q_{1}}, v^{\prime} \in \varsigma_{q_{2}}$, the weight is given by

$$
\sum_{i}\left\langle\left[P_{p_{1}, x} u, P_{p_{2}, x} v\right], \mathbf{e}_{i}\right\rangle\left\langle\left[\mathbf{e}_{i}, P_{q_{2}, x} v^{\prime}\right], P_{q_{1}, x} u^{\prime}\right\rangle=-\left\langle\left[\left[P_{p_{1}, x} u, P_{p_{2}, x} v\right], P_{q_{1}, x} u^{\prime}\right], P_{q_{2}, x} v^{\prime}\right\rangle .
$$

(See Fig. 7a.) The weight as an element of $M_{\Theta(1,1, X)}\left(q_{1}, p_{1}, p_{2}, q_{2} ;-f_{1}, f_{2}, \pm f_{3}\right)$ is

$$
\left\langle\left[\left[P_{q_{1}, x} u^{\prime}, P_{p_{2}, x} v\right], P_{p_{1}, x} u\right], P_{q_{2}, x} v^{\prime}\right\rangle=-\left\langle\left[\left[P_{p_{2}, x} v, P_{q_{1}, x} u^{\prime}\right], P_{p_{1}, x} u\right], P_{q_{2}, x} v^{\prime}\right\rangle .
$$

(This sign in the formula is related to the orientation and is explained in Sect. 7.)

For $M_{\Theta(1,1, X)}\left(p_{1}, q_{1}, q_{2}, p_{2} ; f_{1},-f_{2}, \pm f_{3}\right)$ the weight is

$$
\begin{aligned}
\left\langle\left[\left[P_{p_{1}, x} u, P_{q_{2}, x} \nu^{\prime}\right], P_{q_{1}, x} u^{\prime}\right], P_{p_{2}, x} v\right\rangle & =-\left\langle\left[P_{p_{1}, x} u, P_{q_{2}, x} v^{\prime}\right],\left[P_{p_{2}, x} v, P_{q_{1}, x} u^{\prime}\right]\right\rangle \\
& =+\left\langle\left[P_{p_{1}, x} u,\left[P_{p_{2}, x} v, P_{q_{1}, x} u^{\prime}\right]\right], P_{q_{2}, x} v^{\prime}\right\rangle \\
& =-\left\langle\left[\left[P_{p_{2}, x} v, P_{q_{1}, x} u^{\prime}\right], P_{p_{1}, x} u\right], P_{q_{2}, x} v^{\prime}\right\rangle .
\end{aligned}
$$

And for $M_{\Theta(1,1, X)}\left(q_{1}, p_{1}, q_{2}, p_{2} ;-f_{1},-f_{2}, \pm f_{3}\right)$ we have

$$
-\left\langle\left[\left[P_{p_{1}, x} u, P_{p_{2}, x} v\right], P_{q_{1}, x} u^{\prime}\right], P_{q_{2}, x} v^{\prime}\right\rangle .
$$

For another graph $\mathscr{M}_{\Lambda(1,1, X)}\left(q_{1}, p_{1}, p_{2}, q_{2} ; f_{1}, f_{2}, \pm f_{4}\right)$ the weight is

$$
\sum_{i}\left\langle\left[P_{q_{1}, x} u^{\prime}, P_{p_{1}, x} u\right], \mathbf{e}_{i}\right\rangle\left\langle\left[\mathbf{e}_{i}, P_{p_{2}, x} v\right], P_{q_{2}, x} v^{\prime}\right\rangle=\left\langle\left[\left[P_{q_{1}, x} u^{\prime}, P_{p_{1}, x} u\right], P_{p_{2}, x} v\right], P_{q_{2}, x} v^{\prime}\right\rangle
$$

(Fig. 7b). One can check that the weight for $\mathscr{M}_{\Lambda(1,1, X)}\left(q_{1}, p_{1}, q_{2}, p_{2} ; f_{1},-f_{2}, \pm f_{4}\right)$, etc. is also $\left\langle\left[\left[P_{q_{1}, x} u^{\prime}, P_{p_{1}, x} u\right], P_{p_{2}, x} v\right], P_{q_{2}, x} \nu^{\prime}\right\rangle$.

Then by the Jacobi identity

$$
\left[\left[P_{p_{1}, x} u, P_{p_{2}, x} v\right], P_{q_{1}, x} u^{\prime}\right]+\left[\left[P_{p_{2}, x} v, P_{q_{1}, x} u^{\prime}\right], P_{p_{1}, x} u\right]+\left[\left[P_{q_{1}, x} u^{\prime}, P_{p_{1}, x} u\right], P_{p_{2}, x} v\right]=0 .
$$
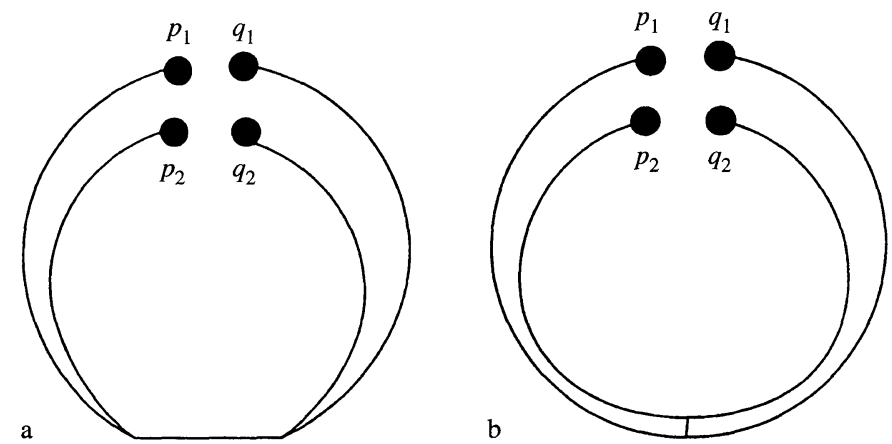

Fig. 7a, b. 
Those terms cancel. To see this we show that in the formula

$$
\begin{aligned}
& \frac{1}{4}\left(\sum_{\varepsilon_{1}, \varepsilon_{2}, \varepsilon_{3}= \pm 1} Z_{\Theta(1,1, X)}\left(\varepsilon_{1} f_{1}, \varepsilon_{2} f_{2}, \varepsilon_{3} f_{3} ; h_{\varepsilon_{1} f_{1}}, g_{\varepsilon_{2} f_{2}}, \varnothing\right)\right. \\
& \left.\quad+\sum_{\varepsilon_{1}, \varepsilon_{2}, \varepsilon_{3}= \pm 1} Z_{\Theta(1, X, 1)}\left(\varepsilon_{1} f_{1}, \varepsilon_{2} f_{2}, \varepsilon_{3} f_{3} ; h_{\varepsilon_{1} f_{1}}, \varnothing g_{\varepsilon_{3} f_{3}}\right)\right) \\
& \quad-\frac{1}{8}\left(\sum_{\varepsilon_{1}, \varepsilon_{2}, \varepsilon_{4}= \pm 1} Z_{\Lambda(1,1, X)}\left(h_{\varepsilon_{1} f_{1}}, g_{\varepsilon_{2} f_{2}}, \varnothing\right)\left(\varepsilon_{1} f_{1}, \varepsilon_{2} f_{2}, \varepsilon_{4} f_{4}\right)\right. \\
& \left.\quad+\sum_{\varepsilon_{1}, \varepsilon_{2}, \varepsilon_{4}= \pm 1} Z_{\Lambda(1, X, 1)}\left(h_{\varepsilon_{1} f_{1}}, \varnothing, g_{\varepsilon_{3} f_{3}}\right)\left(\varepsilon_{1} f_{1}, \varepsilon_{4} f_{4}, \varepsilon_{3} f_{3}\right)\right)
\end{aligned}
$$

the coefficient of each of the terms in (3.11) is $-1 / 2$. In fact, the term $\left[\left[P_{p_{1}, x} u, P_{p_{2}, x} v\right], P_{q_{1}, x} u^{\prime}\right]$ appeared twice in $Z_{\Theta(1,1, X)}(\cdots)$ or $Z_{\Theta(1, X, 1)}(\cdots)$, hence its coefficients is $-1 / 2$. One can show that the coefficient of $\left[\left[P_{p_{2}, x} v, P_{q_{1}, x} u^{\prime}\right], P_{p_{1}, x} u\right]$. On the other hand $\left[\left[P_{q_{1}, x} u^{\prime}, P_{p_{1}, x} u\right], P_{p_{2}, x} v\right]$ appeared 4 times in $Z_{\Lambda(1,1, X)}(\cdots)$ or $Z_{\Lambda(1, X, 1)}(\cdots)$. Therefore its coefficient is again $-1 / 2$. Thus we proved the independence of our invariant of the combinatorial propagator.

\section{Independence of Morse Functions I}

We next consider a one parameter family $\left(\vec{f}_{s}, g_{s}\right) \in\left(C^{\infty}(M)\right)^{3} \times \operatorname{Met}(M)$ and show that $\left(\vec{f}_{0}, g_{0}\right)$ and $\left(\vec{f}_{1}, g_{1}\right)$ give the same invariant. Unfortunately the proof is rather long and technically complicated. It seems that one needs to develop some kinds of homotopical algebra to get rid of the mess in this and the next sections. The reader who is interested in only a basic idea and not so much technical detail may skip the next section.

Before starting the proof let us give an informal explanation of well definedness. One typical way how the Morse function changes is death or birth of critical points. Namely for example we can consider a family

$$
f_{1, s}\left(x_{1}, x_{2}, x_{3}\right)=x_{1}^{3}+s x_{1}-x_{2}^{2}-x_{3}^{2} .
$$

Then a pair of critical points will die at $s=0$. Let $p, q$ be these critical points such that $\eta(p)+\eta(q)=1$. We then find that the combinatorial propagator for $s<0$ has a component $[p] \otimes[q]^{*}$.

Let us assume that there is a family of elements in $\left(x_{s}, Y_{s}, t_{2, s}, t_{3, s}\right) \in$ $\mathscr{M}_{\Theta(1,0,0)}\left(p, q: f_{1, s}, f_{2}, f_{3}\right)$, and try to find what happens when $s=0$. Let's assume moreover that $\left(t_{2, s}, t_{3, s}\right)$ are bounded. Then when $s=0$ we have an element of $\mathscr{M}_{\Theta(1,0,0)}\left(0,0: f_{1,0}, f_{2}, f_{3}\right)$. One can expect after passing zero a new element of $\mathscr{M}_{\Theta(1,0,0)}\left(f_{1, s}, f_{2}, f_{3}\right)$ will be born. Hence our invariant will be the same. To make this argument rigorous is the purpose of this and the next sections.

The proof of invariance of the Morse function is done in two steps. First we assume that Witten complexes are the same for $\left(\vec{f}_{s}, g_{s}\right), s \in[0,1]$, (Sect. 4). Next we conisder the case when the Witten complex changes.

To simplify the notation we assume that $\operatorname{Cr}\left(f_{i, s}\right), i=1,2,3, s \in[0,1]$ are independent of $s$. (We can always do it by using an appropriate diffeomorphism of $M$, since we are assuming that the Witten complex is independent of $s$.) 
We consider the moduli space $\mathscr{M}_{\Theta}(L)$, where $L=\left\{\left(\vec{f}_{s}, g_{s}\right) \mid s \in[0,1]\right\}$. In Sect. 2, we gave a compactification of this moduli space such that $\mathscr{C} \mathscr{M}_{\Theta}(L)-\mathscr{M}_{\Theta}(L)=$ $\mathscr{M}_{\Theta(1,0,0)}(L) \cup \mathscr{M}_{\Theta(0,1,0)}(L) \cup \mathscr{M}_{\Theta(0,0,1)}(L)$. Here $\mathscr{M}_{\Theta(1,0,0)}(L)$ is a moduli space of maps from the graph $\Theta(1,0,0)$ to $M$, such that the two exterior vertices go to the same point in $\operatorname{Cr}\left(f_{1, s}\right)$. This moduli space is of zero dimension. By exactly the same formula we used to define the weight for elements of $\mathscr{M}_{\Theta(1,0,0)}\left(p, q ; f_{1}, f_{2}, f_{3}\right)$, we define $\chi(1, \varsigma): \varsigma_{p} \rightarrow \varsigma_{p}$ for each element $I \in \mathscr{M}_{\Theta(1,0,0)}(L)$. We put

$$
Z_{\Theta(1,0,0)}(L ; \varsigma ; i d, \varnothing, \varnothing)=\sum_{I \in M_{\Theta(1,0,0)}(L)} \pm \operatorname{Tr}(\chi(I, \varsigma)) \text {. }
$$

Then Lemma 2.7 implies that

\section{Lemma 4.1.}

$$
\begin{aligned}
\left(Z_{\Theta}\right. & \left.\left(\vec{f}_{1} ; \varsigma\right)-Z_{\Theta}\left(\vec{f}_{0} ; \varsigma\right)\right)-\left(Z_{\Theta}\left(\vec{f}_{1} ; \varsigma^{\prime}\right)-Z_{\Theta}\left(\vec{f}_{0} ; \varsigma^{\prime}\right)\right) \\
= & Z_{\Theta(1,0,0)}(L ; \varsigma ; i d, \varnothing, \varnothing)-Z_{\Theta(1,0,0)}\left(L ; \varsigma^{\prime} ; i d, \varnothing, \varnothing\right) \\
& +Z_{\Theta(0,1,0)}(L ; \varsigma ; \varnothing, i d, \varnothing)-Z_{\Theta(0,1,0)}\left(L ; \varsigma^{\prime} ; \varnothing, i d, \varnothing\right) \\
& +Z_{\Theta(0,0,1)}(L ; \varsigma ; \varnothing, \varnothing, i d)-Z_{\Theta(0,0,1)}\left(L ; \varsigma^{\prime} ; \varnothing, \varnothing, i d\right) .
\end{aligned}
$$

Proof. Lemma 2.7 implies that $\mathscr{C} \mathscr{M}_{\Theta}(L)$ gives a cobordism between $\mathscr{M}_{\Theta}\left(\vec{f}_{0}\right) \cup$ $-\mathscr{M}_{\Theta}\left(\vec{f}_{1}\right)$ and $\mathscr{M}_{\Theta(1,0,0)}(L) \cup \mathscr{M}_{\Theta(0,1,0)}(L) \cup \mathscr{M}_{\Theta(0,0,1)}(L)$. Since bundles are flat the weights are compatible. The space $\mathscr{C} \mathscr{M}_{\Theta}(L)$ is noncompact but for elements in the end the weights are the same for $\varsigma, \varsigma^{\prime}$. The lemma follows.

We next study the change of the correction terms. First we remark that since we assume that the Witten complex is the same, we can choose the same combinatorial propagator for $\vec{f}_{s}, s \in[0,1]$.

For $p_{1}, q_{1} \in \operatorname{Cr}\left(f_{1}\right)$ with $\eta\left(p_{1}\right)=\eta\left(q_{1}\right)+1$ and $p \in \operatorname{Cr}\left(f_{2}\right)$, we define $\mathscr{M}_{\Theta(1,1,0)}\left(p_{1}, q_{1}, p, p\right)$ for each $\vec{f}_{s}, s \in[0,1]$ in a way similar to Sect. 3. We take its union over all $s$ and denote it by $\mathscr{M}_{\Theta(1,1,0)}\left(L ; p_{1}, q_{1}, p, p\right)$. By a similar transversality argument as before we find that $\mathscr{M}_{\Theta(1,1,0)}\left(L ; p_{1}, q_{1}, p, p\right)$ is a 0 dimensional compact oriented manifold. Also each element $I$ of $\mathscr{M}_{\Theta(1,1,0)}\left(L ; p_{1}, q_{1}, p, p\right)$ induces a map $\chi(I, \varsigma) ; \varsigma_{p} \otimes \varsigma_{p_{1}} \rightarrow \varsigma_{p} \otimes \varsigma_{q_{1}}$. We then define

$$
Z_{\Theta(1,1,0)}\left(L ; \varsigma ; g_{f_{1}}, i d, \varnothing\right)=\sum_{I \in \mathscr{M}_{\Theta(1,1,0)}\left(L ; p_{1}, q_{1}, p\right)} \pm \operatorname{Tr}\left(\chi(I, \varsigma) \circ i d \otimes g\left(p_{1}, q_{1}\right)\right) .
$$

Here $g\left(p_{1}, q_{1}\right)$ is a coefficient of combinatorial propagator.

We define

$\mathscr{S}_{\Theta(1,0,0)}\left(L ; p_{1}, q_{1}\right)=\left\{(x, s) \in M \times[0,1] \mid \begin{array}{l}\lim _{t \rightarrow+\infty} \Phi_{f_{1, s}}^{t}(x)=p_{1} \\ \begin{array}{l}\lim _{t \rightarrow-\infty} \Phi_{f_{1, s}}^{t}(x)=q_{1} \\ \operatorname{grad} f_{2, s}(x) \text { is parallel to grad } f_{3, s}(x)\end{array}\end{array}\right\}$.

In case when $\eta\left(p_{1}\right)=\eta\left(q_{1}\right)+1$ this is a compact oriented 0 dimensional manifold. (In Lemma 3.2 it was $\eta\left(p_{1}\right)=\eta\left(q_{1}\right)+2$. But here we put an extra parameter $s$.) Using this moduli space we define

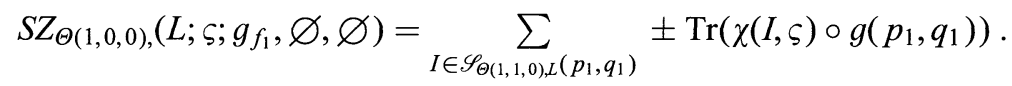

Now in a way similar to Lemma 3.3 we have the following: 


\section{Lemma 4.2.}

$$
\begin{aligned}
& Z_{\Theta(1,0,0)}\left(\vec{f}_{1} ; \varsigma ; g_{f_{1}}, \varnothing, \varnothing\right)-Z_{\Theta(1,0,0)}\left(\vec{f}_{0} ; \varsigma ; g_{f_{1}}, \varnothing, \varnothing\right) \\
& =Z_{\Theta(1,1,0)}\left(L ; \varsigma ; \partial g_{f_{1}}, \varnothing, \varnothing\right)+Z_{\Theta(1,1,0)}\left(L ; \varsigma ; g_{f_{1}}, i d, \varnothing\right) \\
& \quad+Z_{\Theta(1,0,1)}\left(L ; \varsigma ; g_{f_{1}}, \varnothing, i d\right)+S Z_{\Theta(1,0,0)}\left(L ; \varsigma ; g_{f_{1}}, \varnothing, \varnothing\right) .
\end{aligned}
$$

Since $\partial g_{f_{1}}=i d$. we find that the first term of right-hand sides of Lemma 4.2 cancels with the first term of the right-hand side of Lemma 4.1.

To discuss the change of $Z_{\Theta(1,0,0)}\left(\vec{f}_{1} ; \varsigma ; g_{f_{1}}, g_{f_{2}}, \varnothing\right)$ we need to define

$$
\begin{aligned}
& \mathscr{M}_{\Theta(1,1, X)}\left(L ; p_{1}, q_{1} ; p_{2}, q_{2}\right) \\
& =\left\{(x, s) \in M \times[0,1] \mid \begin{array}{l}
\lim _{t \rightarrow+\infty} \Phi_{f_{1, s}}^{t}(x)=p_{1}, \lim _{t \rightarrow-\infty} \Phi_{f_{1, s}}^{t}(x)=q_{1} \\
\lim _{t \rightarrow+\infty} \Phi_{f_{2, s}}^{t}(x)=p_{2}, \lim _{t \rightarrow-\infty} \Phi_{f_{2, s}}^{t}(x)=q_{2}
\end{array}\right\} .
\end{aligned}
$$

If $\eta\left(p_{i}\right)=\eta\left(q_{i}\right)+1$, this space is of 0 dimensional. Using it we define the number $Z_{\Theta(1,1, X)}\left(L ; \varsigma ; g_{f_{1}}, g_{f_{2}}, \varnothing\right)$ in a way similar to Sect. 3 . Then we have

$$
\begin{aligned}
& Z_{\Theta(1,1,0)}\left(\vec{f}_{1}, \varsigma ; g_{f_{1}}, g_{f_{2}}, \varnothing\right)-Z_{\Theta(1,1,0)}\left(\vec{f}_{0} ; \varsigma ; g_{f_{1}}, g_{f_{2}}, \varnothing\right) \\
& =Z_{\Theta(1,1,0)}\left(L, \varsigma ; \partial g_{f_{1}}, g_{f_{2}}, \varnothing\right)+Z_{\Theta(1,1,0)}\left(L, \varsigma ; g_{f_{1}}, \partial g_{f_{2}} \varnothing\right) \\
& \quad+Z_{\Theta(1,1,1)}\left(L, \varsigma ; g_{f_{1}}, g_{f_{2}}, i d\right)+Z_{\Theta(1,1, X)}\left(L, \varsigma ; g_{f_{1}}, g_{f_{2}}, \varnothing\right) .
\end{aligned}
$$

Thus using Lemmas 4.1 and 4.2 we can prove the following lemma in a way similar to Lemma 3.9.

\section{Lemma 4.3.}

$$
\begin{aligned}
& 4\left(\hat{Z}_{\Theta}\left(\vec{f}_{1}, g ; \varsigma\right)-\hat{Z}_{\Theta}\left(\vec{f}_{0}, g ; \varsigma\right)\right)-4\left(\hat{Z}_{\Theta}\left(\vec{f}_{1}, g ; \varsigma^{\prime}\right)-\hat{Z}_{\Theta}\left(\vec{f}_{0}, g ; \varsigma^{\prime}\right)\right) \\
& =\sum_{\varepsilon_{1}, \varepsilon_{2}, \varepsilon_{3}= \pm 1}\left(Z_{\Theta(1,1, X)}\left(\vec{\varepsilon} L ; \varsigma ; g_{\varepsilon_{1} f_{1}}, g_{\varepsilon_{2} f_{2}}, \varnothing\right)+Z_{\Theta(1, X, 1)}\left(\vec{\varepsilon} L ; \varsigma ; g_{\varepsilon_{1} f_{1}}, \varnothing, g_{\varepsilon_{3} f_{3}}\right)\right. \\
& \left.\quad+Z S_{\Theta(1,1,0)}\left(\vec{\varepsilon} L ; \varsigma ; \varnothing, g_{\varepsilon_{2} f_{2}}, g_{\varepsilon_{3} f_{3}}\right)\right)-\sum_{\varepsilon_{1}, \varepsilon_{2}, \varepsilon_{3}= \pm 1}\left(Z_{\Theta(1,1, X)}\left(\vec{\varepsilon} L ; \varsigma^{\prime} ; g_{\varepsilon_{1} f_{1}}, g_{\varepsilon_{2} f_{2}}, \varnothing\right)\right. \\
& \left.\quad+Z_{\Theta(1, X, 1)}\left(\vec{\varepsilon} L ; \varsigma^{\prime} ; g_{\varepsilon_{1} f_{1}}, \varnothing, g_{\varepsilon_{3} f_{3}}\right)+Z S_{\Theta(1,1,0)}\left(\vec{\varepsilon} L ; \varsigma^{\prime} ; \varnothing, g_{\varepsilon_{2} f_{2}}, g_{\varepsilon_{3} f_{3}}\right)\right) .
\end{aligned}
$$

Here we denote $\vec{\varepsilon} L=\left\{\left(\varepsilon_{1} f_{1, s}, \varepsilon_{2} f_{2, s}, \varepsilon_{3} f_{3, s}, g_{s}\right) \mid s \in[0,1]\right\}$. Now the rest of the proof is completely parallel to one of Sect. 3. Namely using the Jacobi identity we find that the right-hand side of Lemma 4.3 cancels with a similar contribution from another graph $\Lambda$. Thus we find

$$
\hat{Z}_{2}\left(\vec{f}_{1}, g ; \varsigma\right)-\hat{Z}_{2}\left(\vec{f}_{0}, g ; \varsigma\right)=\hat{Z}_{2}\left(\vec{f}_{1}, g ; \varsigma^{\prime}\right)-\hat{Z}_{2}\left(\vec{f}_{0}, g ; \varsigma^{\prime}\right)
$$

in the case when the Witten complex does not change for $s \in[0,1]$. 


\section{Independence of Morse Functions II}

Next we consider the case when the critical point set and the boundary operator change. Let $L=\left\{\left(\vec{f}_{s}, g_{s}\right) \in\left(C^{\infty}(M)\right)^{3} \times \operatorname{Met}(M) \mid s \in[0,1]\right\}$ be a generic one parameter family of metrics and functions.

For simplicity we assume that $f_{i, s}$ is independent of $s$ for $i=2,3,4$. Let $C_{*}\left(M ; \varsigma ; f_{1, s}\right)$ be the Witten complex for $f_{1, s}$.

We first recall the proof of the fact that $C_{*}\left(M ; \varsigma ; f_{1,0}\right)$ is chain homotopy equivalent to $C_{*}\left(M ; \varsigma ; f_{1,1}\right)$. (In fact they are both acyclic in our case. But we use the explicit chain homotopy equivalence which (in the general case) is used to show that a chain homotopy type of Witten complex is independent of the choice of Morse function.) Let $p \in \operatorname{Cr}\left(f_{1,0}\right), q \in \operatorname{Cr}\left(f_{1,1}\right)$ such that $\eta(p)=\eta(q)$. We define a moduli space $\overline{\mathscr{M}}_{L}\left(p, q ; f_{1, s}\right)$ as follows. We choose and fix a smooth function $\chi: \mathbf{R} \rightarrow[0,1]$ such that

$$
\chi(s)=0 \text { for } s<-1,
$$

and

$$
\chi(s)=1 \text { for } s>1 .
$$

We put

$$
\mathscr{M}_{L}\left(p, q ; f_{1, s}\right)=\left\{\begin{array}{l|l}
\ell: \mathbf{R} \rightarrow M & \begin{array}{l}
\frac{d \ell}{d t}(t)=-\operatorname{grad}_{\chi(t)} f_{1, \chi(t)}(\ell(t)), \\
\lim _{t \rightarrow+\infty} \ell(t)=p, \\
\lim _{t \rightarrow-\infty} \ell(t)=q
\end{array}
\end{array}\right\} .
$$

Here $\operatorname{grad}_{\chi(t)}$ is the gradient with respect to the metric $g_{\chi(t)}$. We omit this suffix from now on, since the metric $g_{\chi(t)}$ is always used to take the gradient of $f_{1, \chi(t)}$.

$\mathscr{M}_{L}\left(p, q ; f_{1, s}\right)$ is a 0 -dimensional compact oriented manifold. We define $\varphi_{p, q}$ : $\varsigma_{p} \rightarrow \varsigma_{q}$ by

$$
\varphi_{p, q}=\sum_{I \in \mathscr{M}_{L}\left(p, q ; f_{1, s}\right)} \pm P_{\ell} .
$$

We thus obtain $\varphi: C_{*}\left(M ; \varsigma ; f_{1,0}\right) \rightarrow C_{*}\left(M ; \varsigma ; f_{1,1}\right)$.

Lemma 5.1. $\partial_{f_{1,1}} \circ \varphi=\varphi \circ \partial_{f_{1,0}}$.

Proof. Let $\eta(p)=\eta(q)+1$. We obtain a one dimensional moduli space $\mathscr{M}_{L}\left(p, q ; f_{1, s}\right)$ in a similar way. Its boundary is described as:

$\partial \mathscr{M}_{L}\left(p, q ; f_{1}\right)=\left(\overline{\mathscr{M}}\left(p, p^{\prime} ; f_{1,0}\right) \times \overline{\mathscr{M}}_{L}\left(p^{\prime}, q ; f_{1}\right)\right) \cup\left(\overline{\mathscr{M}}_{L}\left(p, q^{\prime} ; f_{1}\right) \times \overline{\mathscr{M}}\left(q^{\prime}, q ; f_{1,1}\right)\right)$.

The lemma follows.

We can prove that $\varphi$ is a chain homotopy equivalence as follows. Using an equation

$$
\frac{d \ell}{d t}(t)=-\operatorname{grad} f_{1, \chi(-t)}(\ell(t))
$$

in a similar way, for $p \in \operatorname{Cr}\left(f_{1,0}\right), q \in \operatorname{Cr}\left(f_{1,1}\right)$, we define a moduli space $\mathscr{M}_{-L}\left(q, p ; f_{1, s}\right)$ and a chain map $\psi: C_{*}\left(M ; \varsigma ; f_{1,1}\right) \rightarrow C_{*}\left(M ; \varsigma ; f_{1,0}\right)$. Chain homotopy between $\psi \varphi$ and the identity is obtained as follows. Choose a sufficiently 
large positive number $S$. We consider the equation

$$
\frac{d \ell}{d t}(t)= \begin{cases}-\operatorname{grad} f_{1, \chi(t+S)}(\ell(t)) & t<-S+1 \\ -\operatorname{grad} f_{1,1}(\ell(t)) & -S+1<t<S-1 \\ -\operatorname{grad} f_{1, \chi(S-t)}(\ell(t)) & t>S-1\end{cases}
$$

Using this equation we have a 0 -dimensional moduli space $\mathscr{M}\left(p, q ; f_{1, s} ; S\right)$ for $p, q \in$ $\operatorname{Cr}\left(f_{1,0}\right)$, with $\eta(p)=\eta(q)$. We find

$$
(\psi \varphi)_{p q}=\sum_{\ell \in \mathscr{M}\left(p, q ; f_{1, s} ; S\right)} \pm P_{\ell} .
$$

The proof of (5.2) is a gluing argument which is now standard in topological field theory. (See [Sc].)

For $r \in[0,1]$ we consider the equation

$$
\frac{d \ell}{d t}(t)= \begin{cases}-\operatorname{grad} f_{1, r \chi(t+S)}(\ell(t)) & t<-S+1 \\ -\operatorname{grad} f_{1, r}(\ell(t)) & -S+1<t<S-1 \\ -\operatorname{grad} f_{1, r \chi(S-t)}(\ell(t)) & t>S-1\end{cases}
$$

Let $\eta(p)=\eta(q)-1$. Using the above equation we obtain a moduli space $\mathscr{M}_{\text {para }}\left(p, q ; f_{1, s}, S\right)$ of 0 dimension. (In fact we need to perturb a bit to achieve transversality. But we omit the discussion about it for simplicity.) Using it we obtain $H_{p q}=\sum_{\ell \in \mathscr{M}_{\mathrm{para}}\left(p, q ; f_{1, s} ; S\right)} \pm P_{\ell}$. This is a map of degree +1 . We have

Lemma 5.3. $H \circ \partial_{f_{1,0}}+\partial_{f_{1,0}} \circ H=1-\psi \varphi$.

We omit the proof.

Now let $g_{f_{1,0}}$ be the combinatorial propagator for $C_{*}\left(M ; f_{1,0} ; \varsigma\right)$. We consider the following moduli space for $p, q \in \operatorname{Cr}\left(f_{1,0}\right)$ :

$\mathscr{M}_{\Theta(1,0,0)}(\vec{f} ; p, q ; S)$

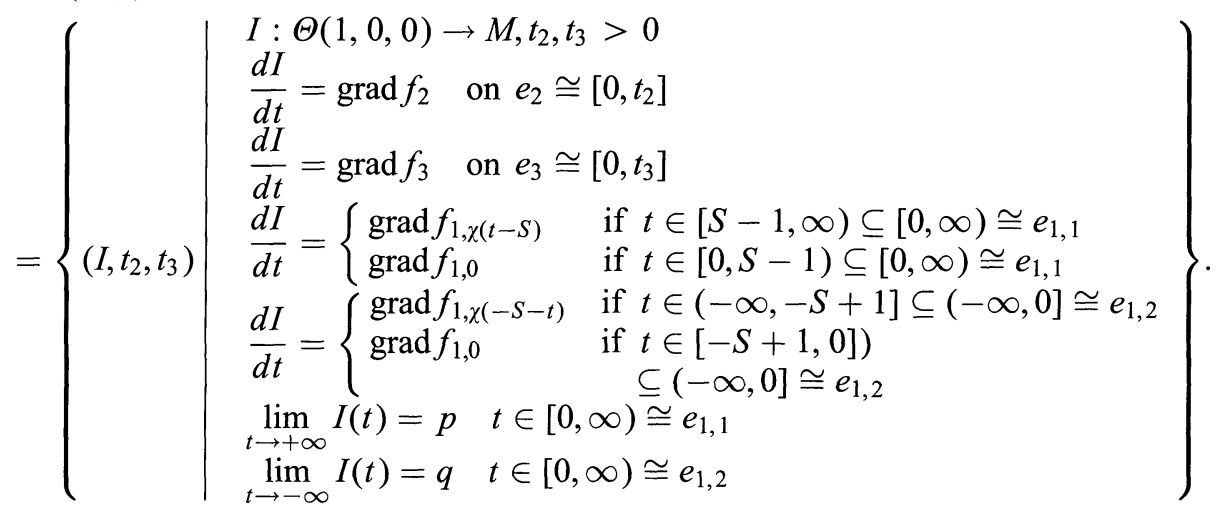

If $\eta(p)=\eta(q)+1$ then $\mathscr{M}_{\Theta(1,0,0)}(\vec{f} ; p, q ; S)$ is a compact 0 dimensional space. Using it and the moduli space $\mathscr{M}_{\Theta(1,0,0)}\left(\vec{f}_{s} ; p, q\right)$ (which we defined in Sect. 1), we define homomorphisms $W(\Theta(1,0,0) ; S): \oplus \varsigma_{p} \rightarrow \oplus \varsigma_{q}$ and $W\left(\Theta_{s}(1,0,0)\right)$ : 
$\oplus \varsigma_{p} \rightarrow \oplus \varsigma_{q}$ as follows:

$$
\begin{aligned}
W_{p, q}(\Theta(1,0,0), S) & =\sum_{I \in \mathscr{M}_{\Theta(1,0,0)}(\vec{f} ; p, q ; S)} \pm \chi(I, \varsigma), \\
W_{p, q}\left(\Theta_{S}(1,0,0)\right) & =\sum_{I \in \mathscr{M}_{\Theta(1,0,0)}\left(\vec{f}_{s} ; p, q\right)} \pm \chi(I, \varsigma) .
\end{aligned}
$$

Let $g_{f_{1,1}}=\sum g_{f_{1,1}}(p, q)[p] \otimes[q]^{*}: \oplus \eta_{q} \rightarrow \oplus \eta_{p}$ be a combinatorial propagator for $f_{1,1}$. We put

$$
Z_{\Theta(1,0,0)}\left(\vec{f}_{1} ; \varsigma ; S ; g_{f_{1,1}}, \varnothing, \varnothing\right)=\operatorname{Tr}\left(g_{f_{1,1}} \circ W(\Theta(1,0,0), S)\right) .
$$

We recall

$$
Z_{\Theta(1,0,0)}\left(\vec{f}_{1} ; \varsigma ; g_{f_{1,1}}, \varnothing, \varnothing\right)=\operatorname{Tr}\left(g_{f_{1,1}} \circ W\left(\Theta_{0}(1,0,0)\right)\right)
$$

Lemma 5.5. For large $S$, we have

$$
W_{p, q}(\Theta(1,0,0), S)=\sum_{p^{\prime}, q^{\prime}} \varphi_{p, p^{\prime}} \circ W_{p^{\prime}, q^{\prime}}\left(\Theta_{0}(1,0,0)\right) \circ \psi_{q^{\prime}, q} .
$$

The lemma follows from the following homeomorphism, which is proved by a usual gluing argument:

$$
\begin{aligned}
\mathscr{M}_{\Theta(1,0,0)}(\vec{f} ; p, q ; S) \cong & \bigcup_{p^{\prime}, q^{\prime} \in \operatorname{Cr}\left(f_{1,0}\right)} \mathscr{M}_{-L}\left(p, p^{\prime} ; f_{1, s}\right) \\
& \times \mathscr{M}_{\Theta(1,0,0)}\left(\vec{f} ; p^{\prime}, q^{\prime}\right) \times \mathscr{M}_{L}\left(q^{\prime}, q ; f_{1, s}\right) .
\end{aligned}
$$

By Lemma 5.5 we have

$$
\begin{aligned}
Z_{\Theta(1,0,0)}\left(\vec{f} ; \varsigma ; S ; g_{f_{1,1}}, \varnothing, \varnothing\right) & =\operatorname{Tr}\left(g_{f_{1,1}} \circ W(\Theta(1,0,0), S)\right) \\
& =\operatorname{Tr}\left(g_{f_{1,1}} \circ \varphi \circ W(\Theta(1,0,0)) \circ \psi\right) \\
& =\operatorname{Tr}\left(\psi \circ g_{f_{1,1}} \circ \varphi \circ W(\Theta(1,0,0))\right) .
\end{aligned}
$$

We next remark that

Lemma 5.7. $\psi \circ g_{f_{1,1}} \circ \varphi+H$ is a combinatorial propagator of $f_{1,0}$.

Proof. By Lemmas 5.1 and 5.3 we have

$$
\begin{aligned}
& \partial_{f_{1,0}} \circ\left(\psi \circ g_{f_{1,1}} \circ \varphi+H\right)+\left(\psi \circ g_{f_{1,1}} \circ \varphi+H\right) \circ \partial_{f_{1,0}} \\
& \quad=\psi \circ\left(\partial_{f_{1,1}} \circ g_{f_{1,1}}+g_{f_{1,1}} \circ \partial_{f_{1,1}}\right) \circ \varphi+\left(\partial_{f_{1,0}} \circ H+H \circ \partial_{f_{1,0}}\right)=1
\end{aligned}
$$

as required.

Since our invariant is independent of the combinatorial propagator, we may choose $g_{f_{1,0}}=\psi \circ g_{f_{1,1}} \circ \varphi+H$.

We thus have

$$
\begin{aligned}
Z_{\Theta(1,0,0)}\left(\vec{f}_{1} ; \varsigma ; S ; g_{f_{1,1}}, \varnothing, \varnothing\right)= & Z_{\Theta(1,0,0)}\left(\vec{f}_{0} ; \varsigma ; g_{f_{1,0}}, \varnothing, \varnothing\right) \\
& -\operatorname{Tr}\left(H \circ W\left(\Theta_{0}(1,0,0)\right)\right)
\end{aligned}
$$


Next, in a way similar to define $\mathscr{M}_{\Theta(1,0,0)}^{\text {para }}(\vec{f} ; p, q ; S)$, we define $\mathscr{M}_{\Theta(1,1,0)}^{\text {para }}\left(\vec{f} ; p_{1}, q_{1} ; p_{2}, q_{2} ; S\right)$ as follows:

\section{$\mathscr{M}_{\Theta(1,1,0)}\left(\vec{f} ; p_{1}, q_{1} ; p_{2}, q_{2} ; S\right)$}

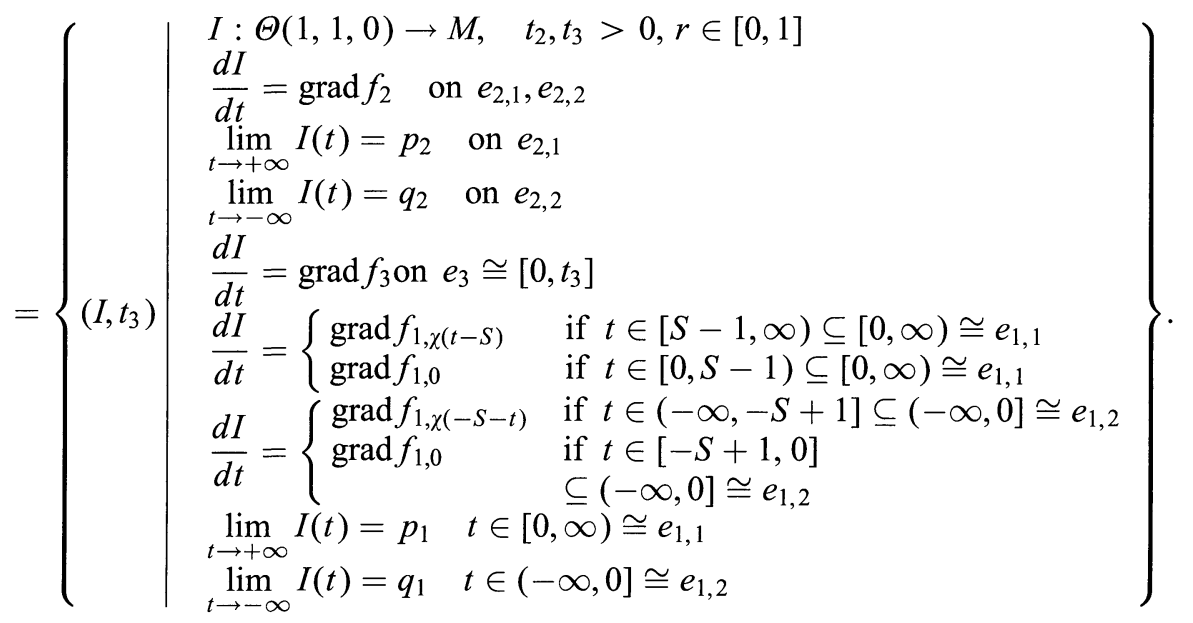

Taking the combinatorial propagator $g_{f_{2}}$ and using the moduli space $\mathscr{M}_{\Theta(1,1,0)}\left(\vec{f}_{1} ; p_{1}, q_{1} ; p_{2}, q_{2} ; S\right)$, we define $W\left(1, g_{f_{2}}, 0\right)$ such that

$$
\begin{aligned}
\left\langle W\left(1, g_{f_{2}}, 0\right)(u), v\right\rangle= & \sum_{p_{1}, q_{1}, p_{2}, q_{2}} \sum_{I \in \mathscr{M}_{\Theta(1,1,0)}\left(\vec{f}_{1} ; p_{1}, q_{1} ; p_{2}, q_{2} ; S\right)} \sum_{i} \\
& \left\langle g_{f_{2}}\left(p_{2}, q_{2}\right) \circ \chi(I, \varsigma) \circ\left(u_{p_{1}} \otimes \mathbf{e}_{i}\right), v_{q_{1}} \otimes \mathbf{e}_{i}\right\rangle .
\end{aligned}
$$

Here $\mathbf{e}_{i}$ is an orthonormal basis of $\varsigma_{p_{2}}$.

Similarly we obtain $W\left(\Theta\left(1, g_{f_{2}}, g_{f_{3}}\right)\right)$ and $W\left(\Theta\left(1,0, g_{f_{3}}\right)\right)$. We can define $Z_{\Theta(1,1,0)}\left(\vec{f}_{1} ; \varsigma ; S ; g_{f_{1,1}}, g_{f_{2}}, \varnothing\right)$ etc. in a similar way. Namely we put

$$
Z_{\Theta(1,1,0)}\left(\vec{f}_{1} ; \varsigma ; S ; g_{f_{1,1}}, g_{f_{2}}, \varnothing\right)=\operatorname{Tr}\left(g_{f_{1,1}} \circ W\left(\Theta\left(1, g_{f_{2}}, 0\right)\right)\right)
$$

and so on. In a way similar to the proof of (5.8.1) we have:

$$
\begin{aligned}
Z_{\Theta(1,1,0)}\left(\vec{f}_{1} ; \varsigma ; S ; g_{f_{1,1}}, g_{f_{2}}, \varnothing\right)= & Z_{\Theta(1,1,0)}\left(\vec{f}_{0} ; \varsigma ; g_{f_{1,0}}, g_{f_{2}}, \varnothing\right) \\
& -\operatorname{Tr}\left(H \circ W\left(\Theta\left(1, g_{f_{2}}, 0\right)\right)\right), \\
Z_{\Theta(1,0,1)}\left(\vec{f}_{1} ; \varsigma ; S ; g_{f_{1,1}}, \varnothing, g_{f_{3}}\right)= & Z_{\Theta(1,1,0)}\left(\vec{f}_{0} ; \varsigma ; g_{f_{1,0}}, \varnothing, g_{f_{3}}\right) \\
& -\operatorname{Tr}\left(H \circ W\left(\Theta\left(1,0, g_{f_{3}}\right)\right)\right), \\
Z_{\Theta(1,1,1)}\left(\vec{f}_{1} ; \varsigma ; S ; g_{f_{1,1}}, g_{f_{2}}, g_{f_{3}}\right)= & Z_{\Theta(1,1,0)}\left(\vec{f}_{0} ; \varsigma ; g_{f_{1,0}}, g_{f_{2}}, g_{f_{3}}\right) \\
& -\operatorname{Tr}\left(H \circ W\left(\Theta\left(1, g_{f_{2}}, g_{f_{3}}\right)\right)\right),
\end{aligned}
$$


etc. We thus found a relation between $\left.Z_{\Theta(}\right)\left(\vec{f}_{1} ; S ; \cdots\right)$ and $\left.Z_{\Theta(}\right)\left(\vec{f}_{0} ; \cdots\right)$. Summing up we got

$$
\begin{aligned}
& -\frac{1}{4} \sum\left(Z_{\Theta(1,0,0)}\left(\vec{\varepsilon} \vec{f}_{1} ; \varsigma ; S ; g_{\varepsilon_{1} f_{1,1}}, \varnothing, \varnothing\right)-Z_{\Theta(1,0,0)}\left(\vec{\varepsilon} \vec{f}_{0} ; \varsigma ; g_{\varepsilon_{1} f_{1,0}}, \varnothing, \varnothing\right)\right) \\
& +\frac{1}{4} \sum\left(Z_{\Theta(1,1,0)}\left(\vec{\varepsilon} \vec{f}_{1} ; \varsigma ; S ; g_{\varepsilon_{1} f_{1,1}}, g_{\varepsilon_{2} f_{2}}, \varnothing\right)-Z_{\Theta(1,1,0)}\left(\vec{\varepsilon} \vec{f}_{0} ; \varsigma ; g_{\varepsilon_{1} f_{1,0}}, g_{\varepsilon_{2} f_{2}}, \varnothing\right)\right) \\
& +\frac{1}{4} \sum\left(Z_{\Theta(1,0,1)}\left(\vec{\varepsilon} \vec{f}_{1} ; \varsigma ; S ; g_{\varepsilon_{1} f_{1,1}}, \varnothing, g_{\varepsilon_{3} f_{3}}\right)-Z_{\Theta(1,0,1)}\left(\vec{\varepsilon} \vec{f}_{1} ; \varsigma ; g_{\varepsilon_{1} f_{1,0}}, \varnothing, g_{\varepsilon_{3} f_{3}}\right)\right) \\
& -\frac{1}{4} \sum\left(Z_{\Theta(1,1,1)}\left(\vec{\varepsilon} \vec{f}_{1} ; \varsigma ; S ; g_{\varepsilon_{1} f_{1,1}}, g_{\varepsilon_{2} f_{2}}, g_{\varepsilon_{3} f_{3}}\right)-Z_{\Theta(1,1,1)}\left(\vec{\varepsilon} \vec{f}_{1} ; \varsigma ; g_{\varepsilon_{1} f_{1,0}}, g_{\varepsilon_{2} f_{2}}, g_{\varepsilon_{3} f_{3}}\right)\right) \\
& +\frac{1}{8} \sum\left(Z_{\Lambda(1,0,1)}\left(\vec{\varepsilon} \vec{f}_{1} ; \varsigma ; S ; g_{\varepsilon_{1} f_{1,1}}, \varnothing, g_{\varepsilon_{3} f_{3}}\right)-Z_{\Lambda(1,0,1)}\left(\vec{\varepsilon} \vec{f}_{1} ; \varsigma ; g_{\varepsilon_{1} f_{1,0}}, \varnothing, g_{\varepsilon_{3} f_{3}}\right)\right) \\
& +\frac{1}{8} \sum\left(Z_{\Lambda(1,1,0)}\left(\vec{\varepsilon} \vec{f}_{1} ; \varsigma ; S ; g_{\varepsilon_{1} f_{1,1}}, g_{\varepsilon_{2} f_{2}}, \varnothing\right)-Z_{\Lambda(1,1,0)}\left(\vec{\varepsilon} \vec{f}_{1} ; \varsigma ; g_{\varepsilon_{1} f_{1,0}}, g_{\varepsilon_{2} f_{2}}, \varnothing\right)\right) \\
& -\frac{1}{8} \sum\left(Z_{\Lambda(1,1,1)}\left(\vec{\varepsilon} \vec{f}_{1} ; \varsigma ; S ; g_{\varepsilon_{1} f_{1,1}}, g_{\varepsilon_{2} f_{2}}, g_{\varepsilon_{4} f_{4}}\right)-Z_{\Lambda(1,1,1)}\left(\vec{\varepsilon} \vec{f}_{1} ; \varsigma ; g_{\varepsilon_{1} f_{1,0}}, g_{\varepsilon_{2} f_{2}}, g_{\varepsilon_{4} f_{4}}\right)\right) \\
& -\frac{1}{8} \sum\left(Z_{\Lambda(1,1,1)}\left(\vec{\varepsilon} \vec{f}_{1} ; \varsigma ; S ; g_{\varepsilon_{1} f_{1,1}}, g_{\varepsilon_{3} f_{3}}, g_{\varepsilon_{4} f_{4}}\right)-Z_{\Lambda(1,1,1)}\left(\vec{\varepsilon} \vec{f}_{1} ; \varsigma ; g_{\varepsilon_{1} f_{1,0}}, g_{\varepsilon_{3} f_{3}}, g_{\varepsilon_{4} f_{4}}\right)\right) \\
& =+\frac{1}{4} \sum \operatorname{Tr}\left(H \circ W_{\vec{\varepsilon} f}(\Theta(1,0,0))\right)-\frac{1}{4} \sum \operatorname{Tr}\left(H \circ W_{\vec{\varepsilon} f}\left(\Theta\left(1, g_{\varepsilon_{2} f_{2}}, 0\right)\right)\right) \\
& -\frac{1}{4} \sum \operatorname{Tr}\left(H \circ W_{\vec{\varepsilon} f}\left(\Theta\left(1,0, g_{\varepsilon_{3} f_{3}}\right)\right)\right)+\frac{1}{4} \sum \operatorname{Tr}\left(H \circ W_{\vec{\varepsilon} f}\left(\Theta\left(1, g_{\varepsilon_{2} f_{2}}, g_{\varepsilon_{3} f_{3}}\right)\right)\right) \\
& -\frac{1}{8} \sum \operatorname{Tr}\left(H \circ W_{\vec{\varepsilon} \vec{f}}\left(\Lambda\left(1,0, g_{\varepsilon_{3} f_{3}}\right)\right)\right)-\frac{1}{8} \sum \operatorname{Tr}\left(H \circ W_{\vec{\varepsilon} f}\left(\Lambda\left(1, g_{\varepsilon_{2} f_{2}}, 0\right)\right)\right) \\
& +\frac{1}{8} \sum \operatorname{Tr}\left(H \circ W_{\vec{\varepsilon} f}\left(\Lambda\left(1, g_{\varepsilon_{4} f_{4}}, g_{\varepsilon_{3} f_{3}}\right)\right)\right)+\frac{1}{8} \sum \operatorname{Tr}\left(H \circ W_{\vec{\varepsilon} f}\left(\Lambda\left(1, g_{\varepsilon_{2} f_{2}}, g_{\varepsilon_{4} f_{4}}\right)\right)\right) \text {. }
\end{aligned}
$$

We next are going to find a relation between $Z_{\Theta(1,0,0)}\left(\vec{f}_{1} ; \varsigma ; g_{f_{1,1}}, \varnothing, \varnothing\right)$ etc. and $Z_{\Theta(1,0,0)}\left(\vec{f}_{1} ; \varsigma ; g_{f_{1,1}}, \varnothing, \varnothing\right)$ etc. For this purpose we use the following moduli space.

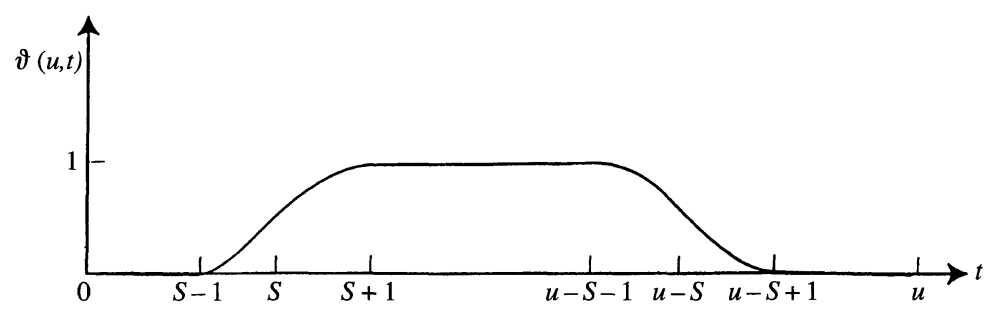

Fig. 8. 
Hereafter we write $Z_{\Theta(1,0,0)}^{\text {para }}\left(\vec{\varepsilon} \vec{f}_{1} ; S ; i d, \varnothing, \varnothing\right)=Z_{\Theta(1,0,0)}^{\text {para }}\left(\vec{\varepsilon} \vec{f}_{1} ; \varsigma, S ; i d, \varnothing, \varnothing\right)-$ $Z_{\Theta(1,0,0)}^{\text {para }}\left(\vec{\varepsilon} \vec{f}_{1} ; \varsigma^{\prime} ; S ; i d, \varnothing, \varnothing\right)$, etc.

$\mathscr{M}_{\Theta(1,0,0)}^{\mathrm{para}}(\vec{f} ; p, q ; S)$

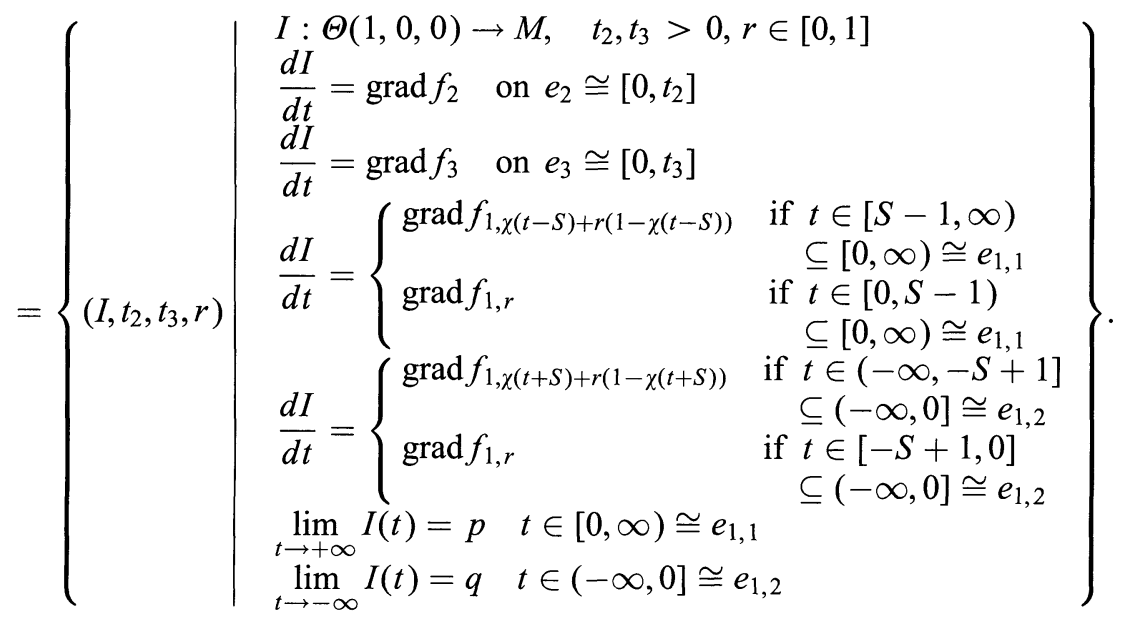

If $\eta(p)=\eta(q)+1$ this moduli space is one dimensional. Its boundary is given as

$$
\begin{aligned}
\partial \mathscr{M}_{\Theta(1,0,0)}^{\text {para }}(\vec{f} ; p, q ; S) & =\mathscr{M}_{\Theta(1,0,0)}(\vec{f} ; p, q ; S) \cup-\mathscr{M}_{\Theta(1,0,0)}\left(\vec{f}_{0} ; p, q\right) \\
& \cup \bigcup_{p^{\prime}} \overline{\mathscr{M}}\left(f_{1,1} ; p, p^{\prime}\right) \times \mathscr{M}_{\Theta(1,0,0)}^{\text {para }}\left(\vec{f} ; p^{\prime}, q ; S\right) \\
& \cup \bigcup_{q^{\prime}} \mathscr{M}_{\Theta(1,0,0)}^{\text {para }}\left(\vec{f} ; p, q^{\prime} ; S\right) \times \overline{\mathscr{M}}\left(f_{1,1} ; q^{\prime}, q\right) \\
& \cup \bigcup_{p_{2} \in \operatorname{Cr}\left(f_{2}\right)} \mathscr{M}_{\Theta(1,1,0)}^{\text {para }}\left(\vec{f} ; p, q ; p_{2}, p_{2} ; S\right) \\
& \cup \bigcup_{p_{2} \in \operatorname{Cr}\left(f_{3}\right)} \mathscr{M}_{\Theta(1,0,1)}^{\text {para }}\left(\vec{f} ; p, q ; p_{3}, p_{3} ; S\right) .
\end{aligned}
$$

It implies

$$
\begin{aligned}
& -Z_{\Theta(1,0,0)}\left(\vec{f} ; \varsigma ; S ; g_{f_{1,1}}, \varnothing, \varnothing\right)+Z_{\Theta(1,0,0)}\left(\vec{f}_{0} ; \varsigma ; g_{f_{1,1}}, \varnothing, \varnothing\right) \\
& =Z_{\Theta(1,0,0)}^{\text {para }}(\vec{f} ; \varsigma ; S ; i d, \varnothing, \varnothing)+Z_{\Theta(1,1,0)}^{\text {para }}\left(\vec{f} ; \varsigma ; S ; g_{f_{1,1}}, i d, \varnothing\right) \\
& \quad+Z_{\Theta(1,1,0)}^{\text {para }}\left(\vec{f} ; \varsigma ; S ; g_{f_{1,1}}, \varnothing, i d\right) .
\end{aligned}
$$

Here we put for example

$$
Z_{\Theta(1,1,0)}^{\mathrm{para}}\left(\vec{f} ; S ; g_{1,1}, i d, \varnothing\right)=\bigcup_{I \in \mathscr{M}_{\Theta(1,1,0)}^{\mathrm{para}}\left(\vec{f} ; p, q: p_{2}, p_{2} ; S\right)} \pm \operatorname{Tr}\left(\left(g_{1,1} \otimes i d\right) \circ \chi(I, \varsigma)\right)
$$


Now we perform a similar cancellation argument to Sect. 3 and obtain the following formula:

$$
\begin{aligned}
& \frac{1}{4} \sum\left(Z_{\Theta(1,0,0)}\left(\vec{\varepsilon} \vec{f}_{1} ; S ; g_{\varepsilon_{1} f_{1,1}}, \varnothing, \varnothing\right)-Z_{\Theta(1,0,0)}\left(\vec{\varepsilon} \vec{f}_{1} ; g_{\varepsilon_{1} f_{1,1}}, \varnothing, \varnothing\right)\right) \\
& -\frac{1}{4} \sum\left(Z_{\Theta(1,1,0)}\left(\vec{\varepsilon} \vec{f}_{1} ; S ; g_{\varepsilon_{1} f_{1,1}}, g_{\varepsilon_{2} f_{2}}, \varnothing\right)-Z_{\Theta(1,1,0)}\left(\vec{\varepsilon} \vec{f}_{1} ; g_{\varepsilon_{1} f_{1,1}}, g_{\varepsilon_{2} f_{2}}, \varnothing\right)\right) \\
& -\frac{1}{4} \sum\left(Z_{\Theta(1,0,1)}\left(\vec{\varepsilon} \vec{f}_{1} ; S ; g_{\varepsilon_{1} f_{1,1}}, \varnothing, g_{\varepsilon_{3} f_{3}}\right)-Z_{\Theta(1,0,1)}\left(\vec{\varepsilon} \vec{f}_{1} ; g_{\varepsilon_{1} f_{1,1}}, \varnothing, g_{\varepsilon_{3} f_{3}}\right)\right) \\
& \left.+\frac{1}{4} \sum\left(Z_{\Theta(1,1,1)}\left(\vec{\varepsilon} \vec{f}_{1} ; S ; g_{\varepsilon_{1} f_{1,1}}, g_{\varepsilon_{2} f_{2}}, g_{\varepsilon_{3} f_{3}}\right)-Z_{\Theta(1,1,1)} \vec{\varepsilon} \vec{f}_{1} ; g_{\varepsilon_{1} f_{1,1}}, g_{\varepsilon_{2} f_{2}}, g_{\varepsilon_{3} f_{3}}\right)\right) \\
& +\frac{1}{8} \sum\left(Z_{\Lambda(1,0,1)}\left(\vec{\varepsilon} \vec{f}_{1} ; S ; g_{\varepsilon_{1} f_{1,1}}, \varnothing, g_{\varepsilon_{3} f_{3}}\right)-Z_{\Lambda(1,0,1)}\left(\vec{\varepsilon} \vec{f}_{1} ; g_{\varepsilon_{1} f_{1,1}}, \varnothing, g_{\varepsilon_{3} f_{3}}\right)\right) \\
& +\frac{1}{8} \sum\left(Z_{\Lambda(1,1,0)}\left(\vec{\varepsilon} \vec{f}_{1} ; S ; g_{\varepsilon_{1} f_{1,1}}, g_{\varepsilon_{2} f_{2}}, \varnothing\right)-Z_{\Lambda(1,1,0)}\left(\vec{\varepsilon} \vec{f}_{1} ; g_{\varepsilon_{1} f_{1,1}}, g_{\varepsilon_{2} f_{2}}, \varnothing\right)\right) \\
& -\frac{1}{8} \sum\left(Z_{\Lambda(1,1,1)}\left(\vec{\varepsilon} \vec{f}_{1} ; S ; g_{\varepsilon_{1} f_{1,1}}, g_{\varepsilon_{2} f_{2}}, g_{\varepsilon_{3} f_{3}}\right)-Z_{\Lambda(1,1,1)}\left(\vec{\varepsilon} \vec{f}_{1} ; g_{\varepsilon_{1} f_{1,1}}, g_{\varepsilon_{2} f_{2}}, g_{\varepsilon_{3} f_{3}}\right)\right) \\
& =-\frac{1}{4} \sum Z_{\Theta(1,0,0)}^{\mathrm{para}}\left(\vec{\varepsilon} \vec{f}_{1} ; S ; i d, \varnothing, \varnothing\right)+\frac{1}{4} \sum Z_{\Theta(1,1,0)}^{\mathrm{para}}\left(\vec{\varepsilon} \vec{f}_{1} ; S ; i d, g_{\varepsilon_{2} f_{2}}, \varnothing\right) \\
& +\frac{1}{4} \sum Z_{\Theta(1,0,1)}^{\mathrm{para}}\left(\vec{\varepsilon} \vec{f}_{1} ; S ; i d, \varnothing, g_{\varepsilon_{3} f_{3}}\right)-\frac{1}{4} \sum Z_{\Theta(1,1,1)}^{\mathrm{para}}\left(\vec{\varepsilon} \vec{f}_{1} ; S ; i d, g_{\varepsilon_{2} f_{2}}, g_{\varepsilon_{3} f_{3}}\right) \\
& +\frac{1}{8} \sum_{\vec{\varepsilon}} Z_{\Lambda(1,1,0)}^{\mathrm{para}}\left(\vec{\varepsilon} \vec{f}_{1} ; S ; i d, g_{\varepsilon_{2} f_{2}}, \varnothing\right)+\frac{1}{8} \sum_{\vec{\varepsilon}} Z_{\Lambda(1,0,1)}^{\mathrm{para}}\left(\vec{\varepsilon} \vec{f}_{1} ; S ; i d, \varnothing, g_{\varepsilon_{3} f_{3}}\right) \text {. } \\
& -\frac{1}{8} \sum_{\vec{\varepsilon}}\left(Z_{\Lambda(1,1,1)}^{\text {para }}\left(\vec{\varepsilon} \vec{f}_{1} ; S ; i d, g_{\varepsilon_{2} f_{2}}, g_{\varepsilon_{4} f_{4}}\right)-\frac{1}{8} \sum_{\vec{\varepsilon}} Z_{\Lambda(1,1,1)}^{\text {para }}\left(\vec{\varepsilon} \vec{f}_{1} ; S ; i d, g_{\varepsilon_{4} f_{4}}, g_{\varepsilon_{3} f_{3}}\right)\right. \text {. }
\end{aligned}
$$

We next study the right-hand side of (5.10). To study the first term, we define the moduli space $\mathscr{M}_{\Theta}\left(\vec{f}_{1} ; S, r\right)$. We choose a function $\vartheta:\{(u, t) \mid t \in[0, u], u>0\} \rightarrow$ $[0,1]$ such that if $u>2 S+10$ we have (Fig. 8)

$$
\vartheta(u, t)=\left\{\begin{array}{ll}
0 & \text { if } t \in[0, S-1] \\
\chi(t-S) & \text { if } t \in[S-1, S+1] \\
1 & \text { if } t \in[S+1, u-2 S-2] \\
\chi(u-S-t) & \text { if } t \in[u-S-1, u-S+1] \\
0 & \text { if } t \in[u-S+1, u]
\end{array} .\right.
$$

(We define $\vartheta$ in the case $u<2 S+10$ also. However the above condition is not assumed in the case $u<2 S+10$.) We then put:

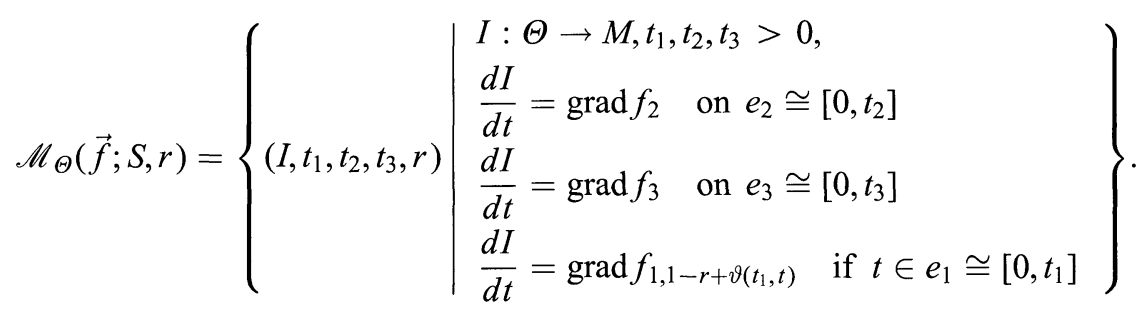


This moduli space has boundaries and ends. The boundary corresponds to $r=$ 1,0 . If $r=0$, this space is equal to $\mathscr{M}_{\Theta}\left(\vec{f}_{1}\right)$.

On the other hand, there are ends of the moduli space $\bigcup_{r \in[0,1]} \mathscr{M}_{\Theta}\left(\vec{f}_{1} ; S, r\right)$ which comes from bubbling of the first edge, namely the case when $t_{1} \rightarrow \infty$. They correspond one to one to the moduli space we used to define $Z_{\Theta(1,0,0)}^{\text {para }}\left(\vec{f}_{1} ; S ; i d, \varnothing, \varnothing\right)$. (We remark that the first edge splits between $S+1$ and $t_{1}-S-1$.)

There are other ends corresponding to $t_{2} \rightarrow \infty, t_{3} \rightarrow \infty$ or $t_{i} \rightarrow 0$. But they will cancel to each other if we consider the other terms. (see Sect. 3,4 and (5.11).)

Using the moduli space of the case $r=1$, namely $\mathscr{M}_{\Theta}\left(\vec{f}_{1} ; S, 1\right)$, we define:

$$
Z_{\Theta}\left(\vec{f}_{1} ; S, 1 ; \varnothing, \varnothing, \varnothing\right)=\sum_{I \in \mathscr{M}_{\Theta}\left(\vec{f}_{1} ; S, 1\right)} \pm \chi(I, \varsigma)
$$

We define $Z_{\Theta(0,1,0)}\left(\vec{f}_{1} ; S, 1 ; \varnothing, g_{f_{2}}, \varnothing\right)$ etc. in a similar way. Then we obtain

$$
\begin{aligned}
& \frac{1}{4} \sum_{\vec{\varepsilon}} Z_{\Theta(1,0,0)}^{\text {para }}\left(\vec{\varepsilon} \vec{f}_{1} ; S ; i d, \varnothing, \varnothing\right)-\frac{1}{4} \sum_{\vec{\varepsilon}} Z_{\Theta(1,1,0)}^{\text {para }}\left(\vec{\varepsilon} \vec{f}_{1} ; S ; i d, g_{\varepsilon_{2} f_{2}}, \varnothing\right) \\
& -\frac{1}{4} \sum_{\vec{\varepsilon}} Z_{\Theta(1,0,1)}^{\text {para }}\left(\vec{\varepsilon} \vec{f}_{1} ; S ; i d, \varnothing, g_{\varepsilon_{3} f_{3}}\right)+\frac{1}{4} \sum_{\vec{\varepsilon}} Z_{\Theta(1,1,1)}^{\text {para }}\left(\vec{\varepsilon} \vec{f}_{1} ; S ; i d, g_{\varepsilon_{2} f_{2}}, g_{\varepsilon_{3} f_{3}}\right) \\
& +\frac{1}{8} \sum_{\vec{\varepsilon}} Z_{\Lambda(1,1,0)}^{\mathrm{para}}\left(\vec{\varepsilon} \vec{f}_{1} ; S ; i d, g_{\varepsilon_{2} f_{2}}, \varnothing\right)+\frac{1}{8} \sum_{\vec{\varepsilon}} Z_{\Lambda(1,0,1)}^{\mathrm{para}}\left(\vec{\varepsilon} \vec{f}_{1} ; S ; i d, \varnothing, g_{\varepsilon_{3} f_{3}}\right) \\
& -\frac{1}{8} \sum_{\vec{\varepsilon}} Z_{\Lambda(1,1,1)}^{\text {para }}\left(\vec{\varepsilon} \vec{f}_{1} ; S ; i d, g_{\varepsilon_{2} f_{2}}, g_{\varepsilon_{4} f_{4}}\right)-\frac{1}{8} \sum_{\vec{\varepsilon}} Z_{\Lambda(1,1,1)}^{\text {para }}\left(\vec{\varepsilon} \vec{f}_{1} ; S ; i d, g_{\varepsilon_{4} f_{4}}, g_{\varepsilon_{3} f_{3}}\right) \\
& =\frac{1}{4}\left(\sum_{\vec{\varepsilon}} Z_{\Theta}\left(\vec{\varepsilon} \vec{f}_{1} ; S, 1 ; \varnothing, \varnothing, \varnothing\right)-\sum_{\vec{\varepsilon}} Z_{\Theta}\left(\vec{\varepsilon} \vec{f}_{1} ; \varnothing, \varnothing, \varnothing\right)\right) \\
& -\frac{1}{4}\left(\sum_{\vec{\varepsilon}} Z_{\Theta(0,1,0)}\left(\vec{\varepsilon} \vec{f}_{1} ; S, 1 ; \varnothing, g_{\varepsilon_{2} f_{2}}, \varnothing\right)-\sum_{\vec{\varepsilon}} Z_{\Theta(0,1,0)}\left(\vec{\varepsilon} \vec{f}_{1} ; \varnothing, g_{\varepsilon_{2} f_{2}}, \varnothing\right)\right) \\
& -\frac{1}{4}\left(\sum_{\vec{\varepsilon}} Z_{\Theta(0,0,1)}\left(\vec{\varepsilon} \vec{f}_{1} ; S, 1 ; \varnothing, \varnothing, g_{\varepsilon_{3} f_{3}}\right)-\sum_{\vec{\varepsilon}} Z_{\Theta(0,0,1)}\left(\vec{\varepsilon} \vec{f}_{1} ; \varnothing, \varnothing, g_{\varepsilon_{3} f_{3}}\right)\right) \\
& +\frac{1}{4}\left(\sum_{\vec{\varepsilon}} Z_{\Theta(0,1,1)}\left(\vec{\varepsilon} \vec{f}_{1} ; S, 1 ; \varnothing, g_{\varepsilon_{2} f_{2}}, g_{\varepsilon_{3} f_{3}}\right)-\sum_{\vec{\varepsilon}} Z_{\Theta(0,1,1)}\left(\vec{\varepsilon} \vec{f}_{1} ; \varnothing, g_{\varepsilon_{2} f_{2}}, g_{\varepsilon_{3} f_{3}}\right)\right) \\
& +\frac{1}{8} \sum_{\vec{\varepsilon}} Z_{\Lambda(0,1,0)}\left(\vec{\varepsilon} \vec{f}_{1} ; S, 1 ; \varnothing, g_{\varepsilon_{2} f_{2}}, \varnothing\right)+\frac{1}{8} \sum_{\vec{\varepsilon}} Z_{\Lambda(0,0,1)}\left(\vec{\varepsilon} \vec{f}_{1} ; S, 1 ; \varnothing, \varnothing, g_{\varepsilon_{3} f_{3}}\right) \\
& -\frac{1}{8}\left(\sum_{\vec{\varepsilon}} Z_{\Lambda(0,1,1)}\left(\vec{\varepsilon} \vec{f}_{1} ; S, 1 ; \varnothing, g_{\varepsilon_{2} f_{2}}, g_{\varepsilon_{4} f_{4}}\right)-\sum_{\vec{\varepsilon}} Z_{\Lambda(0,1,1)}\left(\vec{\varepsilon} \vec{f}_{1} ; \varnothing, g_{\varepsilon_{2} f_{2}}, g_{\varepsilon_{4} f_{4}}\right)\right) \\
& -\frac{1}{8}\left(\sum_{\vec{\varepsilon}} Z_{\Lambda(0,1,1)}\left(\vec{\varepsilon} \vec{f}_{1} ; S, 1 ; \varnothing, g_{\varepsilon_{4} f_{4}}, g_{\varepsilon_{3} f_{3}}\right)-\sum_{\vec{\varepsilon}} Z_{\Lambda(0,1,1)}\left(\vec{\varepsilon} \vec{f}_{1} ; \varnothing, g_{\varepsilon_{4} f_{4}}, g_{\varepsilon_{3} f_{3}}\right)\right) \text {. }
\end{aligned}
$$


We remark terms $\sum_{\vec{\varepsilon}} Z_{\Lambda(0,0,1)}\left(\vec{\varepsilon} \vec{f}_{1} ; S, 1 ; \varnothing, \varnothing, g_{\varepsilon_{3} f_{3}}\right)$ etc. may be nonzero while $\sum_{\vec{\varepsilon}} Z_{\Lambda(0,0,1)}\left(\vec{\varepsilon} \vec{f}_{1} ; \varnothing, \varnothing, g_{\varepsilon_{3} f_{3}}\right)$ must be zero. (The equation we put on the first edge in the definition of the moduli space we use to define $\sum_{\vec{\varepsilon}} Z_{\Lambda(0,0,1)}\left(\vec{\varepsilon} \vec{f}_{1} ; S, 1 ; \varnothing, \varnothing, g_{\varepsilon_{3} f_{3}}\right)$ is a time dependent gradient flow equation. So it may have a nontrivial closed orbit.)

Our next step is to compare the left-hand side of (5.11) to terms related to $\operatorname{Tr}(H \circ W(\Theta(1,0,0)))$, etc. In the following we write $W_{\bar{\varepsilon}}(\Theta(1,0,0))$, etc. in place of $W(\Theta(1,0,0))$, etc. in case we use $\left(\varepsilon_{1} f_{1, s}, \varepsilon_{2} f_{2}, \varepsilon_{3} f_{3}\right)$ to define them.

\section{Lemma 5.12.}

$$
\begin{aligned}
\frac{1}{4} \sum_{\vec{\varepsilon}} Z_{\Theta}\left(\vec{\varepsilon} \vec{f}_{1} ; S, 1 ; \varnothing, \varnothing, \varnothing\right)-\frac{1}{4} \sum_{\vec{\varepsilon}} Z_{\Theta(0,1,0)}\left(\vec{\varepsilon} \vec{f}_{1} ; S, 1 ; \varnothing, g_{\left.\varepsilon_{2} f_{2}, \varnothing\right)}\right. \\
\quad-\frac{1}{4} \sum_{\vec{\varepsilon}} Z_{\Theta(0,0,1)}\left(\vec{\varepsilon} \vec{f}_{1} ; S, 1 ; \varnothing, \varnothing, g_{\varepsilon_{3} f_{3}}\right)+\frac{1}{4} \sum_{\vec{\varepsilon}} Z_{\Theta(0,1,1)}\left(\vec{\varepsilon} \vec{f}_{1} ; S, 1 ; \varnothing, g_{\varepsilon_{2} f_{2}}, g_{\varepsilon_{3} f_{3}}\right) \\
\quad+\frac{1}{8} \sum_{\vec{\varepsilon}} Z_{\Lambda(0,1,0)}\left(\vec{\varepsilon} \vec{f}_{1} ; S, 1 ; \varnothing, g_{\varepsilon_{2} f_{2}}, \varnothing\right)+\frac{1}{8} \sum_{\vec{\varepsilon}} Z_{\Lambda(0,0,1)}\left(\vec{\varepsilon} \vec{f}_{1} ; S, 1 ; \varnothing, \varnothing, g_{\varepsilon_{3} f_{3}}\right) \\
\quad-\frac{1}{8} \sum_{\vec{\varepsilon}} Z_{\Lambda(0,1,1)}\left(\vec{\varepsilon} \vec{f}_{1} ; S, 1 ; \varnothing, g_{\varepsilon_{2} f_{2}}, g_{\varepsilon_{3} f_{3}}\right) \\
=\frac{1}{4}\left(\sum_{\vec{\varepsilon}} Z_{\Theta}\left(\vec{\varepsilon} \vec{f}_{0} ; \varnothing, \varnothing, \varnothing\right)+\sum_{\vec{\varepsilon}} \operatorname{Tr}\left(H \circ W_{\vec{\varepsilon}}(\Theta(1,0,0))\right)\right) \\
\quad-\frac{1}{4}\left(\sum_{\vec{\varepsilon}} Z_{\Theta(0,1,0)}\left(\vec{\varepsilon} \vec{f}_{0} ; \varnothing, g_{\varepsilon_{2} f_{2}}, \varnothing\right)+\sum_{\vec{\varepsilon}} \operatorname{Tr}\left(H \circ W_{\vec{\varepsilon}}\left(\Theta\left(1, g_{\varepsilon_{2} f_{2}}, 0\right)\right)\right)\right) \\
\quad-\frac{1}{4}\left(\sum_{\vec{\varepsilon}} Z_{\Theta(0,0,1)}\left(\vec{\varepsilon} \vec{f}_{0} ; \varnothing, \varnothing, g_{\varepsilon_{3} f_{3}}\right)+\sum_{\vec{\varepsilon}} \operatorname{Tr}\left(H \circ W_{\vec{\varepsilon}}\left(\Theta\left(1,0, g_{\varepsilon_{3} f_{3}}\right)\right)\right)\right) \\
\quad+\frac{1}{4}\left(-\sum_{\vec{\varepsilon}} Z_{\Theta(0,1,1)}\left(\vec{\varepsilon} \vec{f}_{0} ; \varnothing, g_{\varepsilon_{2} f_{2}}, g_{\varepsilon_{3} f_{3}}\right)+\sum_{\vec{\varepsilon}} \operatorname{Tr}\left(H \circ W_{\vec{\varepsilon}}\left(\Theta\left(1, g_{\varepsilon_{2} f_{2}}, g_{\varepsilon_{3} f_{3}}\right)\right)\right)\right) \\
\quad+\frac{1}{8} \sum_{\vec{\varepsilon}} \operatorname{Tr}\left(H \circ W_{\vec{\varepsilon}}\left(\Lambda\left(1, g_{\varepsilon_{2} f_{2}}, 0\right)\right)\right)+\frac{1}{8} \sum_{\vec{\varepsilon}} \operatorname{Tr}\left(H \circ W_{\vec{\varepsilon}}\left(\Lambda\left(1,0, g_{\varepsilon_{3} f_{3}}\right)\right)\right) \\
\quad-\frac{1}{8} \sum_{\vec{\varepsilon}} \operatorname{Tr}\left(H \circ W_{\vec{\varepsilon}}\left(\Lambda\left(1, g_{\varepsilon_{2} f_{2}}, g_{\varepsilon_{4} f_{4}}\right)\right)\right)-\frac{1}{8} \sum_{\vec{\varepsilon}} \operatorname{Tr}\left(H \circ W_{\vec{\varepsilon}}\left(\Lambda\left(1, g_{\varepsilon_{4} f_{4}}, g_{\varepsilon_{3} f_{3}}\right)\right)\right)
\end{aligned}
$$

Before proving Lemma 5.12 we remark that (5.9), (5.10), (5.11) and Lemma 5.12 imply that $\vec{f}_{0}$ and $\vec{f}_{1}$ give the same invariant.

To prove Lemma 5.12 we use the following moduli space which is similar to but different from $\mathscr{M}_{\Theta}\left(\vec{f}_{1} ; S, r\right)$. We choose a function $\Xi:\{(u, t) \mid t \in[0, u]$, $u>0\} \rightarrow[0,1]$ such that if $u>S+T+10$ we have

$$
\Xi(u, t)=\left\{\begin{array}{ll}
0 & \text { if } t \in[0, S-1] \\
\chi(t-T) & \text { if } t \in[T-1, T+1] \\
1 & \text { if } t \in[S+1,2 S-1] \\
\chi(T+S-1-t) & \text { if } t \in[T+S-1, T+S+1] \\
0 & \text { if } t \in[T+S+1, u]
\end{array} .\right.
$$




$$
\mathscr{M}_{\Theta}(\vec{f} ; S, T, r)=\left\{\begin{array}{l|l}
\left(I, t_{1}, t_{2}, t_{3}, r\right) & \begin{array}{l}
I: \Theta \rightarrow M, t_{1}, t_{2}, t_{3}>0 \\
\frac{d I}{d t}=\operatorname{grad} f_{2} \quad \text { on } e_{2} \cong\left[0, t_{2}\right] \\
\frac{d I}{d t}=\operatorname{grad} f_{3} \quad \text { on } e_{3} \cong\left[0, t_{3}\right] \\
\frac{d I}{d t}=\operatorname{grad} f_{1, r \Xi\left(t_{1}, t\right)} \text { if } t \in e_{1} \cong\left[0, t_{1}\right]
\end{array}
\end{array}\right\} .
$$

The space $\bigcup_{r \in[0,1]} \mathscr{M}_{\Theta}(\vec{f} ; S, T, r)$ has boundaries and ends. The boundary is $-\mathscr{M}_{\Theta}(\vec{f} ; S, T, 0) \cup \mathscr{M}_{\Theta}(\vec{f} ; S, T, 1)$. The set $\mathscr{M}_{\Theta}(\vec{f} ; S, T, 0)$ is equal to $\mathscr{M}_{\Theta}\left(\vec{f}_{0}\right)$. We next prove that the ends of $\bigcup_{r \in[0,1]} \mathscr{M}_{\Theta}(\vec{f} ; S, T, r)$ corresponding to the case when $t_{1} \rightarrow \infty$ is equal to $\bigcup_{p, q \in \operatorname{Cr}\left(f_{1,0}\right)} \mathscr{M}_{\Theta(1,0,0)}\left(p, q ; \vec{f}_{0}\right) \times \mathscr{M}_{\text {para }}\left(q, p ; f_{1, s}, S\right)$ if $S$ and $T$ are sufficiently large. Here we recall that $\mathscr{M}_{\text {para }}\left(q, p ; f_{1, s}, S\right)$ is the moduli space we used to define $H$.

To show the behaviour of the end of $\bigcup_{r \in[0,1]} \mathscr{M}_{\Theta}(\vec{f} ; S, T, r)$ described above, we first remark that the ends in question correspond to the following set. (We remark that the first edge splits between $S+T+1$ and $t_{1}$.)

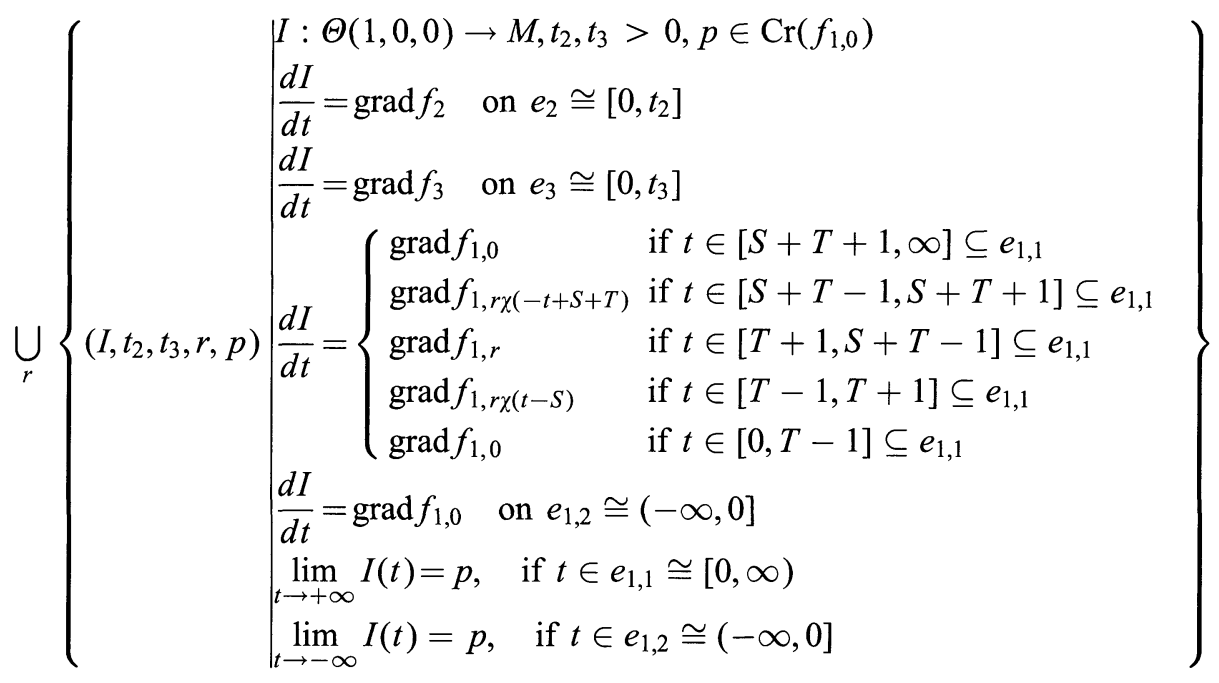

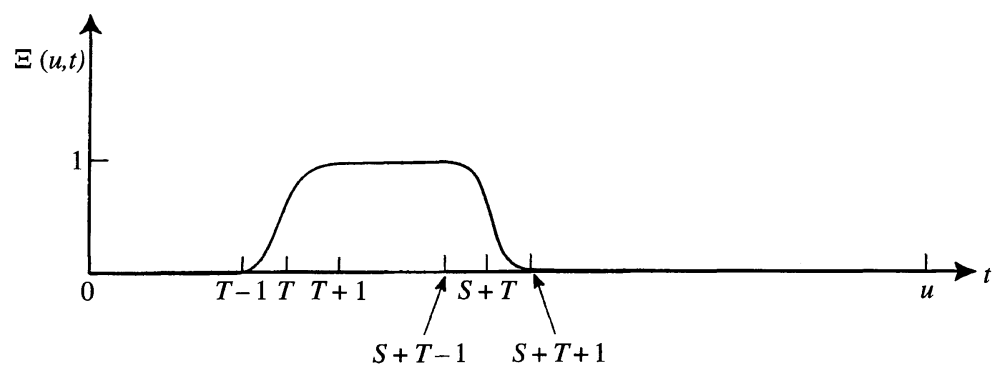

Fig. 9. 
We consider the behavior of this moduli space when $T \rightarrow \infty$. We then find, by a gluing argument, that this space is homeomorphic to $\mathscr{M}_{\Theta(1,0,0)}\left(p, q ; \vec{f}_{0}\right) \times$ $\mathscr{M}_{\text {para }}\left(q, p ; f_{1, s}, S\right)$. Therefore by counting elements of the end of $\bigcup_{r \in[0,1]} \mathscr{M}_{\Theta}(\vec{f} ; S, T, r)$ corresponding to the case when $t_{1} \rightarrow \infty$ with weight, we obtain $\operatorname{Tr}\left(H \circ W_{\vec{\varepsilon}}(\Theta(1,0,0))\right)$.

Thus we obtain the following:

$$
\mathrm{Z}_{\Theta}\left(\vec{\varepsilon} \vec{f}_{1} ; S ; T ; 1 ; \varnothing, \varnothing, \varnothing\right)=\operatorname{Tr}\left(H \circ W_{\vec{\varepsilon}}(\Theta(1,0,0))\right)+\mathrm{Z}_{\Theta}\left(\vec{\varepsilon} \vec{\varepsilon}_{0} ; \varnothing, \varnothing, \varnothing\right) \text {. }
$$

We have a similar formula for graph $\Theta(1,1,0)$, etc. Therefore, to complete the proof of Lemma 5.12, it suffices to prove the following Lemma 5.13. We put

$$
Z_{\Theta}\left(\vec{f}_{1} ; S, T, 1 ; \varnothing, \varnothing, \varnothing\right)=\sum_{I \in \mathscr{M}_{\Theta}\left(\vec{f}_{1} ; S, T, 1\right)} \pm \chi(I, \varsigma) .
$$

Lemma 5.14. If $S$ and $T$ are sufficiently large, then we have

$$
\begin{aligned}
& \frac{1}{4} \sum_{\vec{\varepsilon}} Z_{\Theta}\left(\vec{\varepsilon} \vec{f}_{1} ; S, T, 1 ; \varnothing, \varnothing, \varnothing\right)-\frac{1}{4} \sum_{\vec{\varepsilon}} Z_{\Theta(0,1,0)}\left(\vec{\varepsilon} \vec{f}_{1} ; S, T, 1 ; \varnothing, g_{\varepsilon_{2} f_{2}}, \varnothing\right) \\
& -\frac{1}{4} \sum_{\vec{\varepsilon}} Z_{\Theta(0,0,1)}\left(\vec{\varepsilon} \vec{f}_{1} ; S, T, 1 ; \varnothing, \varnothing, g_{\varepsilon_{3} f_{3}}\right)+\frac{1}{4} \sum_{\vec{\varepsilon}} Z_{\Theta(0,1,1)}\left(\vec{\varepsilon} \vec{f}_{1} ; S, T, 1 ; \varnothing, g_{\varepsilon_{2} f_{2}}, g_{\varepsilon_{3} f_{3}}\right) \\
& +\frac{1}{8} \sum_{\vec{\varepsilon}} Z_{\Lambda(0,1,0)}\left(\vec{\varepsilon} \vec{f}_{1} ; S, T, 1 ; \varnothing, g_{\varepsilon_{2} f_{2}}, \varnothing\right)+\frac{1}{8} \sum_{\vec{\varepsilon}} Z_{\Lambda(0,0,1)}\left(\vec{\varepsilon} \vec{f}_{1} ; S, T, 1 ; \varnothing, \varnothing, g_{\varepsilon_{3} f_{3}}\right) \\
& -\frac{1}{8} \sum_{\vec{\varepsilon}} Z_{\Lambda(0,1,1)}\left(\vec{\varepsilon} \vec{f}_{1} ; S, T, 1 ; \varnothing, g_{\varepsilon_{2} f_{2}}, g_{\varepsilon_{4} f_{4}}\right)-\frac{1}{8} \sum_{\vec{\varepsilon}} Z_{\Lambda(0,1,1)}\left(\vec{\varepsilon} \vec{\varepsilon}_{1} ; S, T, 1 ; \varnothing, g_{\varepsilon_{4} f_{4}}, g_{\varepsilon_{3} f_{3}}\right) \\
& =\frac{1}{4} \sum_{\vec{\varepsilon}} Z_{\Theta}\left(\vec{\varepsilon} \vec{f}_{1} ; S, 1 ; \varnothing, \varnothing, \varnothing\right)-\frac{1}{4} \sum_{\vec{\varepsilon}} Z_{\Theta(0,1,0)}\left(\vec{\varepsilon} \vec{f}_{1} ; S, 1 ; \varnothing, g_{\varepsilon_{2} f_{2}}, \varnothing\right) \\
& -\frac{1}{4} \sum_{\vec{\varepsilon}} Z_{\Theta(0,0,1)}\left(\vec{\varepsilon} \vec{f}_{1} ; S, 1 ; \varnothing, \varnothing, g_{\varepsilon_{3} f_{3}}\right)+\frac{1}{4} \sum_{\vec{\varepsilon}} Z_{\Theta(0,1,1)}\left(\vec{\varepsilon} \vec{f}_{1} ; S, 1 ; \varnothing, g_{\varepsilon_{2} f_{2}}, g_{\varepsilon_{3} f_{3}}\right) \\
& +\frac{1}{8} \sum_{\vec{\varepsilon}} Z_{\Lambda(0,1,0)}\left(\vec{\varepsilon} \vec{f}_{1} ; S, 1 ; \varnothing, g_{\varepsilon_{2} f_{2}}, \varnothing\right)+\frac{1}{8} \sum_{\vec{\varepsilon}} Z_{\Lambda(0,0,1)}\left(\vec{\varepsilon} \vec{f}_{1} ; S, 1 ; \varnothing, \varnothing, g_{\varepsilon_{3} f_{3}}\right) \\
& -\frac{1}{8} \sum_{\vec{\varepsilon}} Z_{\Lambda(0,1,1)}\left(\vec{\varepsilon} \vec{f}_{1} ; S, 1 ; \varnothing, g_{\varepsilon_{2} f_{2}}, g_{\varepsilon_{4} f_{4}}\right)-\frac{1}{8} \sum_{\vec{\varepsilon}} Z_{\Lambda(0,1,1)}\left(\vec{\varepsilon} \vec{f}_{1} ; S, 1 ; \varnothing, g_{\varepsilon_{4} f_{4}}, g_{\varepsilon_{3} f_{3}}\right) \text {. }
\end{aligned}
$$

Proof. We write $\Xi_{S, T}, \vartheta_{S}$ to specify their dependence of $S, T$. We may assume that they depend smoothly on $S$. We define a three parameter family of functions $\aleph_{S, T, A}$ for $S>0, T>S, A \in[0,1]$ such that

$$
\begin{gathered}
\aleph_{S, T, 0}=\Xi_{S, T}, \\
\aleph_{S, T, 1}=\vartheta_{S} .
\end{gathered}
$$

We put $B_{1}=A T+(1-A) S, B_{2}=A(S+T)+(1-A)(u-S)$. Then for $u>$ $T+S+10$ we have

$$
\aleph_{S, T, A}(u, t)= \begin{cases}0 & \text { if } t \in\left[0, B_{1}-1\right] \\ \chi\left(t-B_{1}\right) & \text { if } t \in\left[B_{1}-1, B_{1}+1\right] \\ 1 & \text { if } t \in\left[B_{1}+1, B_{2}-1\right] \\ \chi\left(B_{2}-t\right) & \text { if } t \in\left[B_{2}-1, B_{2}+1\right] \\ 0 & \text { if } t \in\left[B_{2}+1, u\right]\end{cases}
$$


We now put

$$
\mathscr{M}_{\Theta}(\vec{f} ; S, T, A)=\left\{\left(I, t_{1}, t_{2}, t_{3}\right) \mid \begin{array}{l}
I: \Theta \rightarrow M, t_{1}, t_{2}, t_{3}>0, \\
\frac{d I}{d t}=\operatorname{grad} f_{2} \text { on } e_{2} \cong\left[0, t_{2}\right] \\
\frac{d I}{d t}=\operatorname{grad} f_{3} \text { on } e_{3} \cong\left[0, t_{3}\right] \\
\frac{d I}{d t}=\operatorname{grad} f_{1, \aleph_{S, T, A}\left(t_{1}, t\right)} \text { if } t \in e_{1} \cong\left[0, t_{1}\right]
\end{array}\right\} .
$$

This is again a 0 dimensional space for each fixed $S, T$ and $A$. Moving $A \in[0,1]$ we obtain a one dimensional manifold. By (5.14.1) and (5.14.2) its boundary is the union $\mathscr{M}_{\Theta}(\vec{f} ; S, 1) \cup-\mathscr{M}_{\Theta}(\vec{f} ; S, T, 1)$. These moduli spaces are the one we used to define right-and left-hand sides of Lemma 5.13. So to complete the proof of Lemma 5.13 we only need to show that the contribution from the ends of $\bigcup_{A \in[0,1]} \mathscr{M}_{\Theta}(\vec{f} ; S, T, A)$ cancels.

To study the end of $\bigcup_{A \in[0,1]} \mathscr{M}_{\Theta}(\vec{f} ; S, T, A)$ we use the following:

Lemma 5.16. There exists $S_{0}, T_{0}, C$ such that if $S>S_{0}$ then there is no element of $\bigcup_{A \in[0,1]} \mathscr{M}_{\Theta}(\vec{f} ; S, T, A)$ such that $t_{1}>C$, and $C>S_{0}+T_{0}+10$.

Proof. If Lemma 5.16 is false we have a sequence of elements $\left(I_{i}, t_{1, i}, t_{2, i}, t_{3, i}\right)$ of $\mathscr{M}_{\Theta}\left(\vec{f} ; S_{i}, T_{i}, A_{i}\right)$ such that $S_{i} \rightarrow \infty, T_{i} \rightarrow \infty, t_{1, i} \rightarrow \infty$ and $t_{1, i}>S_{i}+T_{i}+10$. By dimension counting it suffices to consider the case when $A_{i}$ converges and $t_{2, i}, t_{3, i}$ converges to some positive number. Then by (5.13.3) $I_{i}$ splits into 2 gradient lines of time dependent gradient vector flow and an element of $\mathscr{M}_{\Theta(1,0,0)}\left(\vec{f}_{0}, p, q\right)$ for some $p, q$. Therefore we have an element of $\mathscr{M}_{\Theta(1,0,0)}\left(\vec{f}_{0}, p, q\right) \times \overline{\mathscr{M}}_{L}\left(q, r ; f_{1, s}\right) \times$ $\overline{\mathscr{M}}_{-L}\left(r, p ; f_{1, s}\right)$. (Remark that the parameter $A$ does not play a role here. Hence we loose one freedom this way. This is the basic reason why the lemma holds.) This space is empty by simple dimension counting. We get a contradiction.

Lemma 5.16 implies that if we choose $S$ sufficiently large, then there is no end of $\bigcup_{A \in[0,1]} \mathscr{M}_{\Theta}(\vec{f} ; S, T, A)$ corresponding to $t_{1} \rightarrow \infty$.

Now we are ready to repeat the argument we did many times. Namely the end coming from $t_{2} \rightarrow \infty, t_{3} \rightarrow \infty$ or $t_{2} \rightarrow 0, t_{3} \rightarrow 0$ cancels after summing them up with other similar terms. The proof of Lemma 5.14 is now complete.

We thus completed the proof of Main Theorems I, II, assuming some lemmas on transversality and orientation.

\section{Compactification of Configuration Space and Transversality at Diagonal}

In this section we discuss the moduli space $\mathscr{S}_{\Theta(1,0,0)}\left(\vec{f}, g ; p_{1}, q_{1}\right)$ we introduced in Sect. 3 and similar moduli spaces which appeared when a non-simply connected subgraph degenerates to a point. To clarify the idea, we consider a more general situation. Probably one can use these ideas to prove the well-definedness of higher loop amplitude.

Let $\Gamma$ be a graph. We assume that each vertex of $\Gamma$ has more than three edges or has only one edge. The vertex with one edge is called an exterior vertex and otherwise it is called an interior vertex. An edge is called an exterior edge if it 
contains an exterior vertex. Otherwise it is called an interior edge. We also fix an orientation to each edge.

Let $\mathscr{T}_{\Gamma}$ be the set of all possible ways to assign a positive number $t_{e}$ to each interior edge $e$.

We associate a Morse function $f_{e}$ to each edge of $\Gamma$. And also we choose a point $p_{v}$ to each exterior vertex of $\Gamma$. We assume that $p_{v}$ is a critical point of the function $f_{e}$, where $e$ is the unique edge containing $v$.

We consider the following moduli space:

$$
\mathscr{M}(\Gamma ; \vec{f}, \vec{p})=\left\{\left(\left(t_{e}\right), I\right) \mid \begin{array}{l}
\left(t_{e}\right) \in \mathscr{T}_{\Gamma} \\
I: \Gamma \rightarrow M \\
\frac{\left.d I\right|_{e}}{d t}=\operatorname{grad} f_{e} \quad \text { on an interior edge } e \cong\left[0, t_{e}\right] \\
\frac{\left.d I\right|_{e}}{d t}=\operatorname{grad} f_{e} \quad \text { on an exterior edge } e \cong(-\infty, 0] \\
\left.\lim _{t \rightarrow-\infty} I\right|_{e}(t)=p_{v} \quad v \in e \text { is an exterior vertex }
\end{array}\right\} .
$$

There is a natural projection $\mathscr{M}(\Gamma ; \vec{f}, \vec{p}) \rightarrow \mathscr{T}_{\Gamma}$. For example if $\Gamma=\Theta$ then $\mathscr{M}(\Theta ; \vec{f})$ is the moduli space $\mathscr{M}_{\Theta}\left(f_{1}, f_{2}, f_{3}\right)$ we studied before.

We put $\mathscr{M}(\Gamma ; V, \vec{p})=\bigcup_{\vec{f} \in V} \mathscr{M}(\Gamma ; \vec{f}, \vec{p})$. Here $V$ is a sufficiently small neighbor-

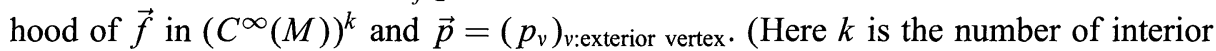
edges.) We choose $V$ so small that the critical point set of $f_{i}^{\prime}$ where $\left(f_{1}^{\prime}, f_{2}^{\prime}, f_{3}^{\prime}\right) \in V$ is identified to the critical point set of $f_{i}$. Then the expression $\mathscr{M}(\Gamma ; \vec{f}, \vec{p})$ makes sense. We have again a map $\mathscr{M}(\Gamma ; \vec{p}) \rightarrow \mathscr{T}_{\Gamma}$.

Lemma 6.1. $\mathscr{M}(\Gamma ; V, \vec{p})$ is a $C^{\infty}$-manifold and $\pi_{\Gamma}: \mathscr{M}(\Gamma ; \vec{p}) \rightarrow \mathscr{T}_{\Gamma}$ is a submersion.

Lemma 6.1 is verified by a straightforward transversality argument similar to one in Sect. 2, where we proved the corresponding transversality result already for the graph we use.

The transversality at the point where one of the numbers is 0 is of different problem. In fact transversality does not hold in the most naive sense. To see this, let us consider

$$
\mathscr{C} \mathscr{T}_{\Gamma}=\left\{\left(t_{e}\right) \mid \begin{array}{l}
e: \text { interior vertex } \\
t_{e} \geqq 0
\end{array}\right\} \cong \mathbf{R}_{\geqq 0}^{c} .
$$

This is a (partial) compactification of $\mathscr{T}_{\Gamma}$. We define $\mathscr{C} \mathscr{M}(\Gamma ; \vec{f}, \vec{p})$ in a way similar to $\mathscr{M}(\Gamma ; \vec{f}, \vec{p})$ but requiring $I(e)=$ point if $t_{e}=0$. There is a natural map $\pi: \mathscr{C} \mathscr{M}(\Gamma ; \vec{f}, \vec{p}) \rightarrow \mathscr{C} \mathscr{T}_{\Gamma} . \mathscr{C} \mathscr{T}_{\Gamma}$ is a manifold with corners. In other words, $\mathscr{C} \mathscr{T}_{\Gamma}$ is a stratified set such that each stratum is a manifold. (We put $\mathscr{C}_{k} \mathscr{T}_{\Gamma}=\left\{\left(t_{e}\right) \in\right.$ $\left.\mathscr{C} \mathscr{T}_{\Gamma} \mid \#\left\{e \mid t_{e}=0\right\}=k\right\}$.) One may ask if each cycle $L \subseteq \mathscr{C}_{\Gamma}$ is transversal to $\pi$ : $\mathscr{C} \mathscr{M}(\Gamma ; \vec{f}, \vec{p}) \rightarrow \mathscr{C} \mathscr{T}_{\Gamma}$. However this is not the case. This is a problem related to the anomaly we discussed. Let us give an example in the case when the dimension of the manifold is 2 (Probably a similar problem occurs in higher dimension.) 
We put

$$
\begin{aligned}
& f_{1}(x, y)=x e^{-y}, \\
& f_{2}(x, y)=x \\
& f_{3}(x, y)=x e^{y} .
\end{aligned}
$$

One can easily see that the moduli space $\mathscr{C} \mathscr{M}(\Theta ; \vec{f})$ is a line $\mathbf{R}$ and the map $\mathscr{M}(\Theta ; \vec{f}) \rightarrow \mathscr{T}_{\Theta} \cong \mathbf{R}_{\geqq 0}^{3}$ hits the origin. (See Fig. 10.)

This picture is quite stable. Namely by perturbing $f_{1}, f_{2}, f_{3}$ we still find a similar situation that is the map $\mathscr{M}(\Theta ; \vec{f}) \rightarrow \mathscr{C} \mathscr{T}_{\Theta} \cong \mathbf{R}_{\geqq 0}^{3}$ still hits the origin. Thus $L=\{(0,0,0)\}$ is not transversal to the map $\pi: \mathscr{C} \mathscr{M}(\Theta ; \vec{f}) \rightarrow \mathscr{C} \mathscr{T}_{\Theta}$.

As we discussed already, to handle a similar problem, we can consider the subsets of the points where gradient lines are parallel, to analyze this problem. To do it more systematically we are going to use compactification of the configuration space similar to one in [FM, Ko]. However, there is a small difference, caused by the fact that we break the symmetry (isomorphism of the graph) by introducing different Morse functions to different edges.

Roughly speaking, we take the real blow up of $\mathscr{C} \mathscr{T}_{\Theta} \cong \mathbf{R}_{\geqq 0}^{3}$ along each $\mathscr{C}_{k} \mathscr{T}_{\Gamma}, k<m$, and obtain $\hat{\mathscr{T}}_{\Gamma}$. More precisely we proceed as follows. We choose and fix a maximal tree $\bar{T} \subseteq \Gamma$. For each edge $e \subseteq \Gamma-\bar{T}$ we choose one of its vertices and attach $e$ to $\bar{T}$ at this vertex. We thus obtain a tree $T$ and a surjection $J: T \rightarrow \Gamma$. Let $C_{0 \text {,int }}(\Gamma)$ be the set of all vertices of $T$ which is mapped to an interior vertex of $\Gamma$ and let $C_{1 \text {,int }}(\Gamma)$ be the set of all interior edges of $\Gamma$. We regard an element of $C_{1 \text {,int }}(\Gamma)$ as an edge of $T$ as well. We say that $A \subseteq C_{1 \text {,int }}(\Gamma)$ is connected if there exists a connected subset of $T$ such that $A$ is the set of all edges contained in it. Our space $\hat{\mathscr{T}}_{\Gamma}$ is a stratified set whose stratum corresponds one to one to the following set $X(\Gamma)$. An element $\mathscr{Z}$ of $X(\Gamma)$ is a set of subsets of $C_{1 \text {,int }}(\Gamma)$ such that

$$
\text { If } A, B \in \mathscr{Z} A \cap B \neq \varnothing \quad \text { then } A \subseteq B \text { or } B \subseteq A \text {. }
$$

If $A \in \mathscr{Z} \quad$ then $A$ is connected

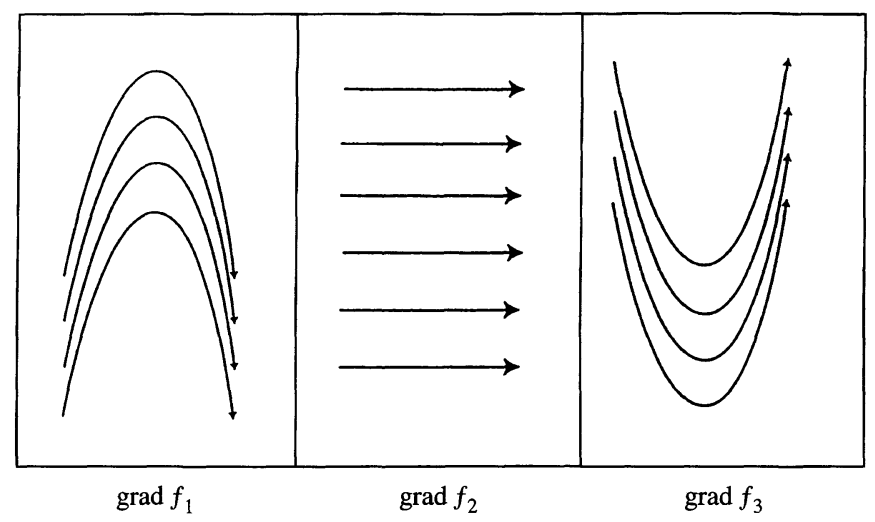

Fig. 10. 
Let $A \in \mathscr{Z}$. We consider the set of all $e \in C_{1, \text { int }}(\Gamma)$ such that $e \in A$ and that if $e \in B \subseteq A, B \in \mathscr{Z}$ then $B=A$. Let $V(A)$ denote this set.

For each $\mathscr{Z} \in X(\Gamma)$ we define $\mathscr{T}(\mathscr{Z})$ as follows:

$$
\mathscr{T}(\mathscr{Z})=\frac{\operatorname{Map}\left(C_{1, \text { int }}(\Gamma) \rightarrow \mathbf{R}_{+}\right)}{\sim_{z}}
$$

We define $\sim_{z}$ as follows. For $t \in \operatorname{Map}\left(C_{1, \text { int }}(\Gamma) \rightarrow \mathbf{R}_{+}\right), r \in \mathbf{R}_{+}$, and $A \in \mathscr{Z}$ we consider

$$
t^{\prime}(e)=\left\{\begin{array}{ll}
t(e) & \text { if } e \notin V(A) \\
r t(e) & \text { if } e \in V(A)
\end{array} .\right.
$$

Then we put $t \sim_{z} t^{\prime}$. We let $\sim_{z}$ be the equivalence relation generated by this relation.

$\hat{\mathscr{T}}_{\Gamma}$ is by definition the union of all $\mathscr{T}(\mathscr{Z})$.

If $\mathscr{Z}=\varnothing$ then $\mathscr{T}(\varnothing)=\mathscr{T}_{\Gamma}$. Hence $\hat{\mathscr{T}}_{\Gamma} \supset \mathscr{T}_{\Gamma}$. The map $\pi: \hat{\mathscr{T}}_{\Gamma} \rightarrow \mathscr{C} \mathscr{T}_{\Gamma}$ is defined as follows:

$$
\pi(t)(e)= \begin{cases}t(e) & \text { if there is no } A \in \mathscr{Z} \text { such that } e \in V(A) \\ 0 & \text { otherwise }\end{cases}
$$

The topology of $\hat{\mathscr{T}}_{\Gamma}$ is defined as follows. Let $t_{i} \in \mathscr{T}_{\Gamma}$. We suppose that $t_{i}(e)$ converges to an element of $\mathbf{R}_{\geqq 0}$ for each $e$. We say that $e^{\prime} \prec e$ if $t_{i}\left(e^{\prime}\right) / t_{i}(e)$ is bounded. We say $e \sim e^{\prime}$ if $e \prec e^{\prime}$ and $e^{\prime} \prec e$. We assume furthermore that $\lim _{i \rightarrow \infty} t_{i}\left(e^{\prime}\right) / t_{i}(e)$ converges to a positive number for each $e \sim e^{\prime}$.

We define $\mathscr{Z}_{0}=\left\{\left\{e \in C_{1 \text {,int }}(\Gamma) \mid e \prec e_{0}\right\} \mid e_{0} \in C_{1 \text {,int }}(\Gamma)\right\}$. We define $\mathscr{Z}$ so that an element of it is a connected component of an element of $\mathscr{Z}_{0}$. For each $A \in \mathscr{Z}$, we choose $e_{0} \in C_{1 \text {,int }}(\Gamma)$ such that

$$
A=A_{e_{0}}=\text { the connected component of }\left\{e \in C_{0}(\Gamma) \mid e \prec e_{0}\right\} \text { containing } e_{0} \in \mathscr{Z} \text {. }
$$

Let $e \in C_{1, \text { int }}(\Gamma)$. Take $e_{0}$ such that $e \in A_{e_{0}}$ and $e \sim e_{0}$. (In other words, $e \in V\left(A_{e_{0}}\right)$.) We put $t_{\infty}(e)=\lim _{i \rightarrow \infty} t_{i}(e) / t_{i}\left(e_{0}\right)$. And we let $\lim _{i \rightarrow \infty} t_{i}$ be the $\sim_{z}$ equivalence class of $t_{\infty}$. We remark that this element (up to equivalence) is independent of the choices of $e_{0}$.

We thus defined the limit of the sequence of elements of $\mathscr{T}_{\Gamma}$. The limit of the elements to $\hat{\mathscr{T}}_{\Gamma}$ is defined in a similar way. Let us describe this set $\hat{\mathscr{T}}_{\Gamma}$ explicitly in the case we need.

First we consider the graph $\Theta(0,0,1)$. Then the tree $T$ is as in Fig. 12 .

$X(\Theta(1,0,0))$ consists of 6 elements $\varnothing,\left\{\left\{e_{1}\right\}\right\},\left\{\left\{e_{2}\right\}\right\},\left\{e_{1}, e_{2}\right\},\left\{\left\{e_{1}\right\}\right.$, $\left.\left\{e_{1}, e_{2}\right\}\right\},\left\{\left\{e_{2}\right\},\left\{e_{1}, e_{2}\right\}\right\}$. The set $\mathscr{T}(\mathscr{Z})$ for each of this four is $\mathbf{R}_{+}^{2}, \mathbf{R}_{+}, \mathbf{R}_{+}$,

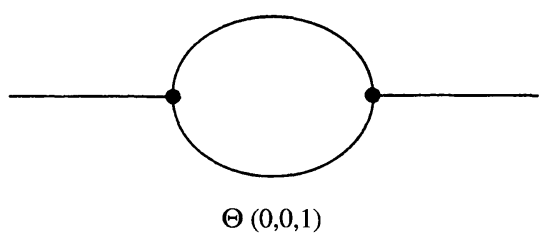

Fig. 11. 


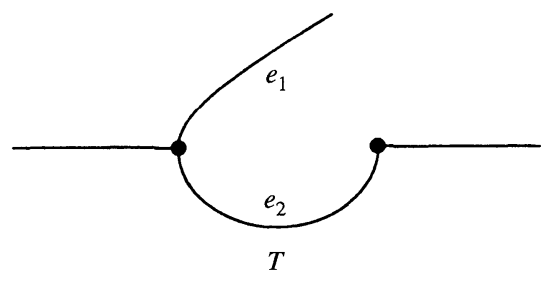

Fig. 12.

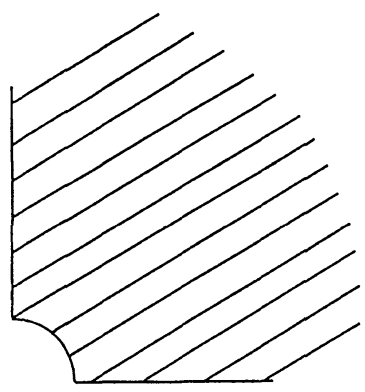

Fig. 13.

$\frac{\left\{\left(t\left(e_{1}\right), t\left(e_{2}\right)\right) \mid t\left(e_{1}\right), t\left(e_{2}\right)>0\right\}}{\mathbf{R}_{+}} \cong(0,1)$, one point and one point, respectively. Gluing them together we obtain the 2 manifold with corners in Fig. 13.

Then $\hat{\mathscr{T}}_{\Theta(0,0,1)} \rightarrow \mathscr{T}_{\Theta(0,0,1)}$ is an isomorphism outside $(0,0)$, and $\pi^{-1}(0,0)$ is identified with an arc.

Next we consider $\Gamma=\Theta$. Our tree $T$ is given by Fig. 14 .

The space $\hat{\mathscr{T}}_{\Theta}$ is given by Fig. 15. In other words, $\pi^{-1}(0,0, t)$ is an arc and $\pi^{-1}(0,0,0)$ is given by Fig. 15 .

We go back to the general case and will construct a space $M(\Gamma)$ for each manifold $M$. This space $M(\Gamma)$ is a compactification of the following configuration space:

$\left\{p: C_{0, \text { int }}(T) \rightarrow M \mid p(v) \neq p\left(v^{\prime}\right)\right.$, if there exists an edge of $T$ joining $v$ and $\left.v^{\prime}\right\}$.

$M(\Gamma)$ is a stratified set. The strata is indexed again by the same set $X(\Gamma)$. Here we regard $X(\Gamma)$ as the set of all $\mathscr{W}$ consisting of subsets of $C_{0, \text { int }}(T)$ satisfying the following conditions:

$$
\text { If } A, B \in \mathscr{W}, A \cap B \neq \varnothing \quad \text { then } A \subseteq B \text { or } B \subseteq A \text {. }
$$

If $A \in \mathscr{W}$ then $A$ is connected. Here we say that $A$ is connected if there exists a connected subcomplex of $T$ such that $A$ is the set of all interior vertices of $T$.

$$
\{v\} \in \mathscr{W} \quad \text { for each } v \in C_{0, \text { int }}(T) .
$$

Let us verify that the set of all such $\mathscr{W}$ is equal to the set of all $Z$ satisfying (6.2). Let $\mathscr{W}$ satisfy (6.3). Let $A \in \mathscr{W}$ with $\# A \geqq 2$. Let $B_{A}$ be the set of all interior edges $e$ such that both of the vertices of $e$ is contained in $A$. We put $\mathscr{Z}(\mathscr{W})$ 


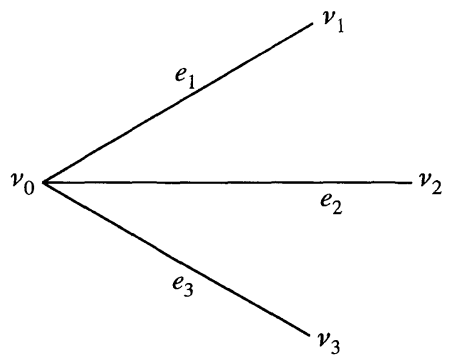

Fig. 14.

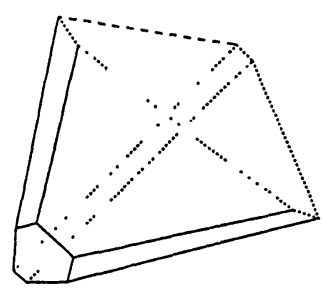

Fig. 15.

$=\left\{B_{A} \mid A \in W, \# A \geqq 2\right\}$. It is easy to see that $\mathscr{W} \rightarrow Z(\mathscr{W})$ is the required one to one correspondence.

Now for $\mathscr{W} \in X(\Gamma)$ we associate a space $M(\mathscr{W})$ as follows. We say that an element $A \in \mathscr{W}$ is maximal if it is maximal with respect to the inclusion. An element of $M(\mathscr{W})$ consists of $p:\{$ all maximal elements of $\mathscr{W}\} \rightarrow M$ and $u(A)$ for each element $A \in \mathscr{W}$. We describe $u(A)$ later. The condition for $p$ is as follows. Let $T(\mathscr{W})$ be the tree obtained from $T$ by shrinking each maximal element to a point. (Here we regard each element $A \in \mathscr{W}$ as a connected subcomplex.) Then $p$ associates a point of $M$ to each vertex of $T(\mathscr{W})$. We assume that $p(v) \neq p\left(v^{\prime}\right)$ for each of the vertices of $T(\mathscr{W})$ which are joined by an edge.

We next describe $u(A)$. First we consider the case when $A$ is maximal. We consider all elements $A(\mathscr{W})$ of $\mathscr{W}$ contained in $A$. $(A \notin A(\mathscr{W})$.) Let us take all elements of $A(\mathscr{W})$ which are maximal among elements of $A(\mathscr{W})$. Let $A_{m}(\mathscr{W})$ be this set. Then

$$
u(A) \in \frac{\operatorname{Map}\left(A_{m}(\mathscr{W}) \rightarrow T_{p(A)} M\right)}{\sim} .
$$

Here we say $u_{1}(A) \sim u_{2}(A)$ if there exists $r \in \mathbf{R}_{+}$and $u \in T_{p(A)} M$ such that $u_{1}(A)(B)=r u_{2}(A)(B)+u$ for each $B \in A_{m}(\mathscr{W})$.

Next let $B \in A_{m}(\mathscr{W})$. We define $B(\mathscr{W})$ and $B_{m}(\mathscr{W})$ in the same way. Then

$$
u(B) \in \frac{\operatorname{Map}\left(B_{m}(\mathscr{W}) \rightarrow T_{u(A)(B)}\left(T_{p(A)}(M)\right)\right)}{\sim} .
$$

Here $\sim$ is defined in a similar way. We remark that if $u_{1}(A) \sim u_{2}(A)$ then there is a canonical isomorphism $\left.\left.T_{u_{1}(A)(B)}\left(T_{p(A)}(M)\right)\right) \cong T_{u_{2}(A)(B)}\left(T_{p(A)}(M)\right)\right)$.

We continue in the same way and define $u(C)$ for each $C \in \mathscr{W}$. The space $M(\mathscr{W})$ is the correction of all such $(p, u)$. We remark that if $\mathscr{W}_{0}=\{\{v\} \mid v \in$ 


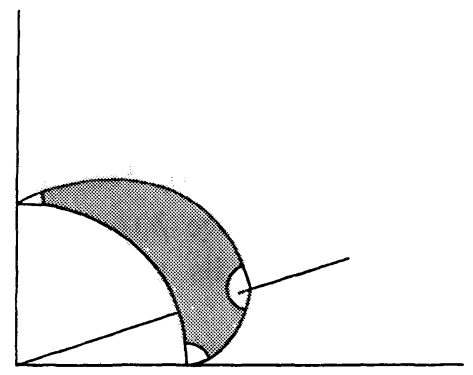

Fig. 16.

$\left.C_{0, \text { int }}(\Gamma)\right\}$ then

$$
M\left(\mathscr{W}_{0}\right)=\left\{p: C_{0, \text { int }}(T) \rightarrow M \mid p(v) \neq p\left(v^{\prime}\right),\right.
$$

if there exists an edge of $T$ joining $v$ and $\left.v^{\prime}\right\}$.

We have an obvious topology on $M(\mathscr{W})$ so that $M(\mathscr{W})$ is a smooth manifold. We put $M(\Gamma)=\bigcup_{\mathscr{W} \in X(\Gamma)} M(\mathscr{W})$. We define a topology on it as follows. For simplicity we define a limit of the sequence $p_{i} \in M\left(\left\{\{v\} \mid v \in C_{0, \text { int }}(\Gamma)\right\}\right)$ only. We assume that $p_{i}(v)$ converges for each $v \in C_{0, \text { int }}(\Gamma)$. Let $p_{\infty}(v)$ be its limit.

We first define $\mathscr{W} \in X(\Gamma)$. For each $v \in C_{0 \text {,int }}(\Gamma)$ we consider the set of all $v^{\prime} \in C_{0, \text { int }}(\Gamma)$ such that $p_{\infty}(v)=p_{\infty}\left(v^{\prime}\right)$. Let $A_{v}$ be the connected component of this set containing $v$. A maximal element of $\mathscr{W}$ is $A_{v}$ for some $v \in C_{0, \text { int }}(\Gamma)$.

We identify $A_{v}$ with a connected subcomplex of $\Gamma$. Let $C_{1, \text { int }}\left(A_{v}\right)$ be the set of all interior edges of this subcomplex. Let $e, e^{\prime} \in C_{1, \text { int }}\left(A_{v}\right)$. Let $v_{1}(e)$ and $v_{2}(e)$ be the vertices of this edge. We say $e \prec e^{\prime}$ if $d\left(p_{i}\left(v_{1}(e)\right), p_{i}\left(v_{2}(e)\right) / d\left(p_{i}\left(v_{1}\left(e^{\prime}\right)\right), p_{i}\left(v_{2}\left(e^{\prime}\right)\right)\right.\right.$ is bounded as $i \rightarrow \infty$. Here $d$ is a distance function for a Riemannian metric of $M$. (We fix it but the construction is independent of it.) We let $A_{v, e}$ be the connected component of $\left\{e^{\prime} \in C_{1, \text { int }}\left(A_{v}\right) \mid e^{\prime} \prec e\right\}$ containing $e$. $A_{v, e}$ may be regarded as a connected subcomplex of $A_{v}$. We define $A_{v}(\mathscr{W})$ by

$$
A_{v}(\mathscr{W})=\left\{A_{v, e} \mid e \in C_{1, \text { int }}\left(A_{v}\right)\right\} \cup\left\{\left\{v^{\prime}\right\} \mid v^{\prime} \in A_{v}\right\} .
$$

We thus defined $\mathscr{W}$. We define $p_{\infty}:\{$ all maximal elements of $\mathscr{W}\} \rightarrow M$ by $p_{\infty}\left(A_{v}\right)=p_{\infty}(v)$.

Let $A_{v}$ be a maximal element of $\mathscr{W}$. We next define $u\left(A_{v}\right):\left(A_{v}\right)_{m}(\mathscr{W}) \rightarrow$ $T_{p_{\infty}\left(A_{v}\right)}(M)$. We remark that $p_{\infty}\left(A_{v}\right)=p_{\infty}(v)$. We fix elements $v_{0}, v \in A_{v}$, such that if $v, v_{0} \in A^{\prime}, A^{\prime} \in \mathscr{W}$ then $A^{\prime}=A_{v}$. For $v^{\prime} \in B, B \in\left(A_{v}\right)_{m}(\mathscr{W})$, we set

$$
u\left(A_{v}\right)(B)=\lim _{i \rightarrow \infty}\left(\frac{\exp _{p_{i}(v)}^{-1}\left(p_{i}\left(v^{\prime}\right)\right)}{d\left(p_{i}(v), p_{i}\left(v_{0}\right)\right)}\right) \in T_{p_{\infty}(v)}(M)
$$

in case the right-hand side converges. We remark that $u\left(A_{v}\right)(B)$ is independent of the choice of $v^{\prime} \in B$ and the $\sim_{\mathscr{W}}$ equivalence class of $u\left(A_{v}\right)$ is independent of the choice of $v$ and $v_{0}$.

Next let $B \in\left(A_{v}\right)_{m}(\mathscr{W})$. We define $u(B): B_{m}(\mathscr{W}) \rightarrow T_{u(B)}\left(T_{p_{\infty}(v)} M\right)$. We fix $v^{\prime}, v_{0}^{\prime} \in B$ such that $v^{\prime}, v_{0}^{\prime} \in B^{\prime} B^{\prime} \in \mathscr{W}, B^{\prime} \subseteq B$ imply $B^{\prime}=B$. Let $C \in B_{m}(\mathscr{W})$. We 
choose $v^{\prime \prime} \in C$ and put

$$
\tilde{p}_{i}(C)=\left(D \exp _{p_{i}(v)}\right)^{-1}\left(\exp _{p_{t}\left(v^{\prime}\right)}^{-1}\left(v^{\prime \prime}\right)\right) .
$$

Here $\exp _{p_{l}\left(v^{\prime}\right)}^{-1}\left(v^{\prime \prime}\right) \in T_{p_{t}\left(v^{\prime}\right)}(M)$, and $D \exp _{p_{l}(v)}: T_{\exp _{p_{l}(v)}^{-1}\left(p_{t}\left(v^{\prime}\right)\right)}\left(T_{p_{i}(v)}(M)\right) \rightarrow T_{p_{t}\left(v^{\prime}\right)} M$ is a differential of the exponential map. Hence $\tilde{p}_{i}(C)=\left(D \exp _{p_{t}(v)}\right)^{-1}\left(\exp _{p_{i}\left(v^{\prime}\right)}^{-1}\left(v^{\prime \prime}\right)\right)$ is an element of $T_{\exp _{p i(v)}^{-1}\left(p_{t}\left(v^{\prime}\right)\right)}\left(T_{p_{l}(v)} M\right)$. Its norm is almost equal to $d\left(p_{i}\left(v^{\prime}\right)\right.$, $\left.p_{i}\left(v^{\prime \prime}\right)\right)$. We put

$$
u(B)(C)=\lim _{i \rightarrow \infty}\left(\frac{\tilde{p}_{i}(C)}{d\left(p_{i}\left(v^{\prime}\right), p_{i}\left(v_{0}^{\prime}\right)\right)}\right) \in T_{\exp _{p_{l}(v)}^{-1}\left(p_{l}\left(v^{\prime}\right)\right)}\left(T_{p_{l}(v)} M\right)
$$

if the right-hand side converges. Again this limit is independent of $v^{\prime \prime} \in C$. And the equivalence class of $u(B)$ is independent of $v^{\prime}, v_{0}^{\prime}$.

We continue in this way and define $u$ for each element of $\mathscr{W}$.

We thus described the topology of $M(\Gamma)=\bigcup_{\mathscr{W} \in X(\Gamma)} M(\mathscr{W})$. We can prove that $M(\Gamma)$ is a smooth manifold with corners.

We are going to define a map $\Phi: M \times \hat{\mathscr{T}}_{\Gamma} \times\left(C^{\infty}(M)\right)^{m} \times$ Met $\rightarrow M(\Gamma)$. This map is a generalization of one we defined at the beginning of Sect. 2. Here $m$ is the number of interior edges. We remark that both $M(\Gamma)$ and $\hat{\mathscr{T}}_{\Gamma}$ are stratified sets. Their strata both correspond one to one to the set $X(\Gamma)$. Our map $\Phi$ respects this stratification. We are going to define $\Phi$ for each stratum.

In case $Z=\varnothing$ which corresponds to $\mathscr{W}=\left\{\{v\} \mid v \in C_{0, \text { int }}(\Gamma)\right\}$ our definition is as follows. We first recall

$$
M(\mathscr{W})=\left\{p: C_{0, \text { int }}(T) \rightarrow M \mid p(v) \neq p\left(v^{\prime}\right),\right.
$$

if there exists an edge of $T$ joining $v$ and $\left.v^{\prime}\right\}$

and

$$
\mathscr{T}(\mathscr{Z})=\operatorname{Map}\left(C_{1, \text { int }}(\Gamma) \rightarrow \mathbf{R}_{+}\right)
$$

in this case. We fix an interior vertex $v_{0}$. Then for each $v \in C_{0, \text { int }}(T)$ there is a unique minimal path $\ell_{v}$ joining it to $v_{0}$. We put $\ell_{v}=e_{1, v} \cup \cdots \cup e_{k(v), v}$ such that $v_{0} \in e_{1, v}, e_{i, v} \cap e_{i+1, v}=$ one point, and $v \in e_{k(v), v}$. Then for $t \in \mathscr{T}(\mathscr{Z})=$ $\operatorname{Map}\left(C_{1, \text { int }}(\Gamma) \rightarrow \mathbf{R}_{+}\right)$we put

$$
\Phi\left(p, t,\left(f_{e}\right), g\right)(v)=\left(\Phi_{f_{e_{k}(v), v}}^{t\left(e_{k(v), v}\right)} \circ \cdots \circ \Phi_{f_{e_{1, v}}}^{t\left(e_{1, v}\right)}\right)(p) .
$$

(We recall that $\Phi_{f_{e_{1, v}}}^{t\left(e_{1, v}\right)}$ is the exponential map of the vector field grad $f_{e_{1, v}}$.)

We next consider the case when $\mathscr{Z}=\left\{A_{i}\right\}$ such that $A_{i} \cap A_{j}=\varnothing$ for $i \neq j$. This corresponds to the case $\mathscr{W}=\left\{A_{i}\right\} \cup\left\{\{v\} \mid v \in C_{0 \text {,int }}(\Gamma)\right\}$. (Here $A_{i}$ is a connected subcomplex of $T$, which is regarded both as a subset of $C_{1, \text { int }}(\Gamma)$ and $C_{0, \text { int }}(T)$.) Let $\mathscr{T}(\mathscr{Z})$ be the tree obtained by shrinking each of the elements of $\mathscr{Z}=\left\{A_{i}\right\}$ to a point. An element $t$ of $\mathscr{T}(Z)$ determines an element of $\operatorname{Map}\left(C_{1, \text { int }}(\mathscr{T}(\mathscr{Z})) \rightarrow \mathbf{R}_{+}\right)$. Then in the same way as above we obtain $p:\{$ all maximal elements of $\mathscr{W}\} \rightarrow M$. (Remark that the vertex of $T_{\mathscr{Z}}$ corresponds to a maximal element of $\mathscr{W}$.) Next we determine $u\left(A_{i}\right) . t$ also determines an element of $t_{i}: C_{1, \text { int }}\left(A_{i}\right) \rightarrow \mathbf{R}_{+}$(up to an equivalence $t_{i} \sim r \cdot t_{i}$ ). Since $T$ is a tree there is a unique vertex $v_{i}$ of $A_{i}$ which is closest from $v_{0}$. For $v \in C_{0, \text { int }}\left(A_{i}\right)$ there is a unique minimal path $\ell_{i, v}$ in $A_{i}$ joining 
$v$ to $v_{i}$. Let $\ell_{i, v}=e_{i, 1, v} \cup \cdots \cup e_{i, k_{i}(v), v}$ such that $v_{i} \in e_{i, 1, v}, e_{i, j, v} \cap e_{i, j+1, v}=$ one point, $v \in e_{i, k_{t}(v), v}$. We put

$$
u\left(A_{i}\right)(v)=\sum_{j} t\left(e_{i, j, v}\right) \operatorname{grad} f_{e_{l, j, v}} .
$$

We then define $\Phi\left(p, t,\left(f_{e}\right), g\right)=(t, u)$.

The definition of $\Phi$ for general strata is similar.

Lemma 6.4. The map $\Phi: M \times \hat{\mathscr{T}}_{\Gamma} \times\left(C^{\infty}(M)\right)^{m} \times \operatorname{Met} \rightarrow M(\Gamma)$ is a smooth map between manifolds with corners. Its restriction to each stratum is a submersion.

The lemma is a direct consequence of the definition and the straightforward transversality argument.

Now we can use Lemma 6.4 to construct a compactification of the moduli space $\mathscr{M}(\Gamma ; \vec{f} \vec{p})$. We first consider the submanifold of

$$
\begin{aligned}
& M(\varnothing)=\left\{p: C_{0}, \operatorname{int}(T) \rightarrow M \mid p(v) \neq\left(v^{\prime}\right),\right. \\
& \text { if there exists an edge of } \left.T \text { joining } v \text { and } v^{\prime}\right\} .
\end{aligned}
$$

We fix Morse functions $f_{e}$ for each exterior edge $e$ of $\Gamma$. And we fix a critical point $p_{v}$ for each exterior vertex $v$. We consider the set $Y$ of all elements of $M(\varnothing)$ such that

If $v^{\prime}$ is an interior vertex joined by $e$ to a exterior vertex $v$. Then $p\left(v^{\prime}\right)$ is contained in the unstable manifold of $p_{v}$ of the gradient vector field grad $f_{e}$

$$
\text { If } v, v^{\prime} \in C_{0, \text { int }}(T) \text { such that } J(v)=J\left(v^{\prime}\right) \text {, then } p(v)=p\left(v^{\prime}\right) \text {. }
$$

The following lemma is immediate from the definition.

Lemma 6.6. $\Phi^{-1}(Y) \cap\left(M \times \mathscr{T}_{\Gamma} \times\left\{\left(f_{e}\right)\right\} \times\{g\}\right)$ is equal to $\mathscr{M}(\Gamma ; \vec{f}, \vec{p})$. Here the component of $\vec{f}$ is $f_{e}$ in the above formula for interior edge $e$ and is $f_{e}$ in (6.5.1) for exterior edge $e$.

We next use the following:

Lemma 6.7. For generic $f_{e}$, the closure $\bar{Y}$ of $Y$ in $M(\Gamma)$ is a manifold with corners. For each strata $M(\mathscr{W})$ of $M(\Gamma)$ the codimension of $M(\mathscr{W}) \cap \bar{Y}$ depends only on the Morse index of $p_{v}$ and the combinatorial type of the graph and is independent of $\mathscr{W}$.

Proof. Let $(p, u) \in M(\mathscr{W})$. We describe the condition for this element in $\bar{Y}$.

Let $A$ be a maximal element of $\mathscr{W}$. The condition for $p(A) \in M$ is

If $v^{\prime}$ is an interior vertex joined by $e$ to an exterior vertex $v$ and if $v^{\prime} \in A$, then $p(A)$ is contained in the stable manifold of $p_{v}$ of the gradient vector field grad $f_{e}$.

Next we consider a pair $v, v^{\prime}$ of vertices of $T$ such that for $J(v)=J\left(v^{\prime}\right)$ we put conditions for such pairs. Let us describe those conditions. Let $A, A^{\prime} \in \mathscr{W}$ be the maximal element such that $v \in A$ and $v^{\prime} \in A^{\prime}$. If $A \neq A^{\prime}$ then we assume

$$
p(A)=p\left(A^{\prime}\right)
$$


If $A=A^{\prime}$ we need to put conditions on $u$. Let $B, B^{\prime}$ be the maximal element of $A(\mathscr{W})$ such that $v \in B$ and $v^{\prime} \in B^{\prime}$. If $B \neq B^{\prime}$ then we put

$$
u(B)=u\left(B^{\prime}\right) .
$$

If $B=B^{\prime}$ we choose the maximal elements of $B(\mathscr{W})$ containing $v, v^{\prime}$. We continue in this way. It is straightforward to see that these conditions describe $\bar{Y}$.

The co-dimension for each stratum is found from these descriptions. Namely we put $\operatorname{dim} M$ conditions for each pair $v, v^{\prime}$ of vertices of $T$ such that $J(v)=J\left(v^{\prime}\right)$. (Note that condition (6.8) is applied once for each such pair).

The number of conditions corresponding to each exterior edge is the Morse index and is independent of the stratum.

The proof of Lemma 6.7 is complete.

Using Lemma 6.7 we obtain:

Lemma 6.9. $\Phi^{-1}(\bar{Y})$ is a manifold with corners. The projection $\Phi^{-1}(\bar{Y}) \rightarrow \mathscr{T}_{\Gamma} \times$ $\left(C^{\infty}(M)\right)^{m} \times$ Met is a Fredholm map. This projection respects stratification. The index of the restriction to each stratum is the same.

The tarnsversality we used in earlier sections follows immediately from Lemma 6.9.

\section{Orientation}

In this section we define an orientation of our moduli spaces and verify compatibility of them in various contexts. We first define an orientation of $\mathscr{M}_{\Theta}\left(f_{1}, f_{2}, f_{3}\right)$. We regard it as an intersection of three submanifolds of dimension 4 in $M^{2}$, as we discussed in introduction. Namely we put

$$
M(f)=\left\{\left(p, \Phi_{f}^{t}(p)\right) \mid p \in M, t>0\right\} \subseteq M^{2} .
$$

We regard it as an image of the map $\Psi_{f}: M \times \mathbf{R}_{+} \rightarrow M^{2},(p, t) \mapsto\left(p, \Phi_{f}^{t}(p)\right)$. Then an orientation of $M$ induces one on $M(f)$. We recall $\mathscr{M}_{\Theta}\left(f_{1}, f_{2}, f_{3}\right)=$ $M\left(f_{1}\right) \cap M\left(f_{2}\right) \cap M\left(f_{3}\right)$. Hence we define a sign for each point on $\mathscr{M}_{\Theta}\left(f_{1}, f_{2}, f_{3}\right)$ induced from the orientation of $M^{2}$ and $M\left(f_{i}\right)$. Thus $\mathscr{M}_{\Theta}\left(f_{1}, f_{2}, f_{3}\right)$ is an oriented 0-dimensional manifold.

We remark that there is a diffeomorphism $\tau: \mathscr{M}_{\Theta}\left(f_{1}, f_{2}, f_{3}\right) \rightarrow \mathscr{M}_{\Theta}\left(-f_{1},-f_{2}\right.$, $\left.-f_{3}\right),\left(p, q ; t_{1}, t_{2}, t_{3}\right) \mapsto\left(q, p ; t_{1}, t_{2}, t_{3}\right)$.

Lemma 7.1. $\tau$ is an orientation preserving diffeomorphism.

We recall that we took the sum over $\mathscr{M}_{\Theta}\left(\varepsilon_{1} f_{1}, \varepsilon_{2} f_{2}, \varepsilon_{3} f_{3}\right)$. Hence if $\tau$ were orientation reversing then our invariant would be always 0 .

Proof of Lemma 7.1. Let $J: M \times \mathbf{R}_{+} \rightarrow M \times \mathbf{R}_{+}$be an orientation preserving diffeomorphism $(p, t) \mapsto\left(\Phi_{f}^{-1}(p), t\right)$. And $R: M \times M \rightarrow M \times M,(p, q) \mapsto(q, p)$ be an orientation reversing diffeomorphism. We remark that $\Psi_{-f}=R \circ \Psi_{f} \circ J$. Hence the lemma is a consequence of the following:

Sublemma 7.2. Let $V_{i}, i=1,2,3$ be oriented linear subspaces of an oriented vector space $V$ and $R: V \rightarrow V$ be an orientation reversing isomorphism. Orientations of 
$V_{i}$ induces one on $R\left(V_{i}\right)$. Then $\left.R\right|_{V_{1} \cap V_{2} \cap V_{3}}: V_{1} \cap V_{2} \cap V_{3} \rightarrow R\left(V_{1}\right) \cap R\left(V_{2}\right) \cap R\left(V_{3}\right)$ is orientation preserving.

Proof. One finds that $\left.R\right|_{V_{1} \cap V_{2}}: V_{1} \cap V_{2} \rightarrow R\left(V_{1}\right) \cap R\left(V_{2}\right)$ is orientation reversing. Hence $\left.R\right|_{V_{1} \cap V_{2} \cap V_{3}}$ is orientation preserving, as required.

We next turn to the orientation of $\mathscr{M}_{\Theta(1,0,0)}\left(p, q ; f_{1}, f_{2}, f_{3}\right)$, etc. We consider stable and unstable manifolds. Namely:

$$
\begin{aligned}
& U(p)=\left\{x \in M \mid \lim _{t \rightarrow-\infty} \Phi_{f}^{t}(x)=p\right\}, \\
& S(p)=\left\{x \in M \mid \lim _{t \rightarrow+\infty} \Phi_{f}^{t}(x)=p\right\} .
\end{aligned}
$$

We remark that

$$
\mathscr{M}_{\Theta(1,0,0)}\left(p, q ; f_{1}, f_{2}, f_{3}\right)=(S(p) \times U(q)) \cap M\left(f_{2}\right) \cap M\left(f_{3}\right) .
$$

Therefore orientations of stable and unstable manifolds determines orientation of $\mathscr{M}_{\Theta(1,0,0)}\left(p, q ; f_{1}, f_{2}, f_{3}\right)$. The most natural way to define orientation of stable and unstable manifolds is to modify the definition of the Witten complex a bit as follows:

$$
C_{k}(M ; f ; \varsigma)=\frac{\bigoplus_{\begin{array}{c}
p \in \operatorname{Cr}(f), \eta(p)=k \\
\text { an orientation of } S(p)
\end{array}} \varsigma_{p} \bullet[p, \varepsilon]}{\sim} .
$$

Here $[p, \varepsilon] \sim-[p,-\varepsilon]$. Then the orientation of $\mathscr{M}\left(\left[p, \varepsilon_{p}\right],\left[q, \varepsilon_{q}\right]\right)$ is automatically fixed. Namely the orientation $\varepsilon$ of $S(p)$ and the orientation of $M$ determines an orientation of $U(p)$. Therefore the orientation of $\mathscr{M}\left(\left[p, \varepsilon_{p}\right],\left[q, \varepsilon_{q}\right]\right)=S(p) \cap U(q)$ will be fixed. Using this orientation and induced orientation on $\overline{\mathscr{M}}\left(\left[p, \varepsilon_{p}\right],\left[q, \varepsilon_{q}\right]\right)$ we can determine the sign in the definition of $\partial_{p q}$.

Also we can determine the orientation of $\mathscr{M}_{\Theta(1,0,0)}\left(\left[p, \varepsilon_{p}\right],\left[q, \varepsilon_{q}\right] ; f_{1}, f_{2}, f_{3}\right)$ $=(S(p) \times U(q)) \cap M\left(f_{2}\right) \cap M\left(f_{3}\right)$.

We next discuss the compatibility of this orientation to one for $\mathscr{M}_{\Theta}\left(f_{1}, f_{2}, f_{3}\right)$. Let us consider a one parameter family $L$ of $(\vec{f}, g)$. We fix an orientation of $L$. Then $\mathscr{M}_{\Theta}(L)=\bigcup_{\left(f_{1}, f_{2}, f_{3} ; g\right) \in L} \mathscr{M}_{\Theta}\left(f_{1}, f_{2}, f_{3}\right)$ is an oriented one dimensional manifold. One of its boundary components is given as $\bigcup_{\substack{\left.\left(f_{1}, f_{2}, f_{3}, g\right) \in L \\ 1 \in C \in C_{1}\right)}} \mathscr{M}_{\Theta(1,0,0)}\left(p, p ; f_{1}, f_{2}, f_{3}\right)$. As before we can define orientation of $\bigcup_{\substack{\left(1_{1}, f_{2}, f_{3} ; g\right) \in L \\ p \in \operatorname{Cr}\left(f_{1}\right)}} \mathscr{M}_{\Theta(1,0,0)}\left(p, p ; f_{1}, f_{2}, f_{3}\right)$. To prove that these orientations are compatible we only need to prove the following:

Lemma 7.3. $\bigcup_{\substack{\left(\mathcal{f}_{1}, f_{2}, f_{3}, g\right) \in L \\ p \in \operatorname{Cr}\left(f_{1}\right)}} S(p) \times U(p)$ is a boundary component of $\bigcup_{\left(f_{1}, f_{2}, f_{3} ; g\right) \in L} M(f)$. Their orientations are compatible.

Proof. The problem is local so we only need to work in a neighborhood of a critical point $p$ of $f_{1, s}$. Then by using the Morse lemma, we may assume that $f$ is a standard quadratic function. In that case the lemma is easy to verify by looking at the spaces directly.

We need to verify compatibility of the orientation in various contexts. First compatibility of orientation in Lemma 3.1 is an immediate consequence of Lemma 7.3. 
More interesting is the statement on orientation for Lemma 3.8. Let us state it more precisely.

We consider two moduli spaces $\bigcup_{\left(f_{1}, f_{2}, f_{3} ; g\right) \in L} \mathscr{M}_{\Theta(1,0,0)}\left(p, q ; f_{1}, f_{2}, f_{3}\right)$ and $\bigcup_{\left(f_{1}, f_{2}, f_{3} ; g\right) \in L} \mathscr{M}_{\Theta(1,0,0)}\left(q, p ;-f_{1}, f_{2}, f_{3}\right)$. Their closures both contain

$$
\mathscr{S}(1,0,0)\left(p_{1}, q_{1} ; f_{1}, f_{2}, f_{3}\right)=\mathscr{S}(1,0,0)\left(q_{1}, p_{1} ;-f_{1}, f_{2}, f_{3}\right)
$$

We are going to compare orientations attached to each term of the element of $\mathscr{S}(1,0,0)\left(p_{1}, q_{1} ; f_{1}, f_{2}, f_{3}\right)=\mathscr{S}(1,0,0)\left(q_{1}, p_{1} ;-f_{1}, f_{2}, f_{3}\right)$.

Lemma 7.4. The orientations of $\mathscr{S}(1,0,0)\left(p_{1}, q_{1} ; f_{1}, f_{2}, f_{3}\right)$ and $\mathscr{S}(1,0,0)\left(q_{1}, p_{1}\right.$; $\left.-f_{1}, f_{2}, f_{3}\right)$ are opposite to each other.

Proof. We put

$$
\mathscr{S}\left(f_{2}, f_{3}\right)=\left\{(x, x) \in M^{2} \mid \operatorname{grad} f_{2}(x) \text { is parallel to } \operatorname{grad} f_{3}(x)\right\}
$$

$\mathscr{S}\left(f_{2}, f_{3}\right)$ is a component of the boundary of $M\left(f_{2}\right) \cap M\left(f_{3}\right)$, and hence is an oriented manifold. We remark that $\mathscr{S}\left(f_{2}, f_{3}\right)$ is contained in the diagonal. We have

$$
\begin{gathered}
\mathscr{S}(1,0,0)\left(p_{1}, q_{1} ; f_{1}, f_{2}, f_{3}\right)=\left(S_{f_{1}}\left(p_{1}\right) \times U_{f_{1}}\left(q_{1}\right)\right) \cap \mathscr{S}\left(f_{2}, f_{3}\right), \\
\mathscr{S}(1,0,0)\left(q_{1}, p_{1} ;-f_{1}, f_{2}, f_{3}\right)=\left(S_{-f_{1}}\left(q_{1}\right) \times U_{-f_{1}}\left(p_{1}\right)\right) \cap \mathscr{S}\left(f_{2}, f_{3}\right) .
\end{gathered}
$$

We remark that $S_{f_{1}}\left(q_{1}\right)$ is the same manifold as $U_{-f_{1}}\left(q_{1}\right)$. We may assume that their orientations coincide. $S_{f_{1}}\left(p_{1}\right) \times U_{f_{1}}\left(q_{1}\right) \rightarrow U_{f_{1}}\left(q_{1}\right) \times S_{f_{1}}\left(p_{1}\right)=S_{-f_{1}}\left(q_{1}\right) \times U_{-f_{1}}\left(p_{1}\right)$ is an orientation preserving diffeomorphism. The lemma then follows from the fact that if $R: V \rightarrow V$ is orientation reversing, and $R: V_{i} \rightarrow R\left(V_{i}\right)$ is orientation preserving, then $\left.R\right|_{V_{1} \cap V_{2}}: V_{1} \cap V_{2} \rightarrow R\left(V_{1}\right) \cap R\left(V_{2}\right)$ is orientation reversing.

We next turn to the compatibility of the orientation we used at the end of Sect. 3. Namely we prove:

Lemma 7.5. $\mathscr{M}_{\Theta(1,1, X)}\left(p_{1}, q_{1}, p_{2}, q_{2}\right)\left(f_{1}, f_{2}, f_{3}\right)$ and $\mathscr{M}_{\Theta(1,1, X)}\left(q_{1}, p_{1}, p_{2}, q_{2}\right)\left(-f_{1}, f_{2}\right.$, $f_{3}$ ) has an opposite orientation to each other.

Proof. Let $\Delta \subseteq M^{2}$ be the diagonal. Then

$$
\mathscr{M}_{\Theta(1,1, X)}\left(p_{1}, q_{1}, p_{2}, q_{2}\right)\left(f_{1}, f_{2}, f_{3}\right)=\left(S_{f_{1}}\left(p_{1}\right) \times U_{f_{1}}\left(q_{1}\right)\right) \cap\left(U_{f_{2}}\left(p_{2}\right) \times S_{f_{2}}\left(q_{2}\right)\right) \cap \Delta,
$$

$\mathscr{M}_{\Theta(1,1, X)}\left(q_{1}, p_{1}, p_{2}, q_{2}\right)\left(f_{1}, f_{2}, f_{3}\right)=\left(S_{-f_{1}}\left(q_{1}\right) \times U_{-f_{1}}\left(p_{1}\right)\right) \cap\left(U_{f_{2}}\left(p_{2}\right) \times S_{f_{2}}\left(q_{2}\right)\right) \cap \Delta$.

The lemma therefore follows from the same argument as Lemma 7.1.

There are many other cases for which we need to verify the compatibility of orientations. But the argument for them are the same as those we discussed in this section already. 


\section{Concluding Remarks}

As we mentioned in the introduction, we conjecture that the invariant discussed in this paper coincides with Chern-Simons Perturbation theory by Axelrod-Singer, etc. We here discuss a heuristic argument to "show" this conjecture.

We first recall the definition of the 2-loop amplitude of Chern-Simons Perturbation theory briefly. Let $\varsigma$ be a flat $g$ bundle on a compact oriented 3 manifold $M$ such that $H^{*}(M ; \varsigma)=0$. Let $\Omega^{k}(M ; \varsigma)$ be the set of all smooth $k$-forms on $M$ with a $\varsigma$ coefficient and let $d_{k}: \Omega^{k}(M ; \varsigma) \rightarrow \Omega^{k+1}(M ; \varsigma)$ be the exterior derivative. Since our bundle $\varsigma$ is flat we have $d_{k+1} d_{k}=0$. The assumption $H^{*}(M ; \varsigma)=0$ is then equivalent to $\operatorname{ker} d_{k}=\operatorname{Im} d_{k-1}$.

We fix a Riemannian metric on $M$ and consider an orthonormal complement $\left(\operatorname{ker} d_{k}\right)^{\perp}$ of $\operatorname{ker} d_{k}=\operatorname{Im} d_{k-1}$. The restriction of $d_{k}$ to $\left(\operatorname{ker} d_{k}\right)^{\perp}$ is an isomorphism: $\left(\operatorname{ker} d_{k}\right)^{\perp} \rightarrow \operatorname{ker} d_{k+1}$. Let $d_{k}^{-1}$ be its inverse.

We consider the bundle $\operatorname{Hom}\left(\pi_{l}^{*} \varsigma, \pi_{2}^{*} \varsigma\right)$ on $M^{2}$. Here $\pi_{i}: M^{2} \rightarrow M$ is the projection to the $i^{\text {th }}$ component.

Definition 8.1. A section $P \in \Gamma\left(M^{2} \backslash \Delta ; \operatorname{Hom}\left(\pi_{2}^{*} \varsigma, \pi_{1}^{*} \varsigma\right) \otimes \Lambda^{2}\left(M^{2}\right)\right)$ is said to be a propagator if

$$
d_{k}^{-1}(u)(x)=\int_{y \in M} P(x, y) \wedge u(y) d y
$$

holds for each $u \in \operatorname{ker} d_{k+1}$.

We next define the $\Theta$-trace: $\operatorname{End}(\mathfrak{g})^{\otimes 3} \rightarrow \mathbf{R}$ for each semi simple Lie algebra $\mathfrak{g}$. Let $\mathbf{e}_{i}$ be an orthonormal basis of $\mathfrak{g}$. We put

$$
\operatorname{Tr}_{\Theta}\left(u_{1} \otimes u_{2} \otimes u_{3}\right)=\sum_{i, j, k}\left\langle\left[e_{i}, e_{j}\right], e_{k}\right\rangle\left\langle\left[u_{1}\left(e_{i}\right), u_{2}\left(e_{j}\right)\right], u_{3}\left(e_{k}\right)\right\rangle .
$$

Now the leading term of the 2 loop amplitude of Chern-Simons Perturbation theory is given by

$$
\int_{(x, y) \in M^{2}} \operatorname{Tr}_{\Theta}(P(x, y) \wedge P(x, y) \wedge P(x, y)) .
$$

We recall here that $P(x, y) \wedge P(x, y) \wedge P(x, y) \in \Lambda_{(x, y)}^{6}\left(M^{2}\right) \otimes \operatorname{Hom}\left(\varsigma_{x}, \varsigma_{y}\right)^{\otimes 3}$. Hence $\operatorname{Tr}_{\Theta}(P(x, y) \wedge P(x, y) \wedge P(x, y)) \in \Lambda_{(x, y)}^{6}\left(M^{2}\right)$. So (8.2) gives a number. It is proved in $[\mathrm{AS}]$ that (8.2) together with correction terms (terms related to the other graph $\Lambda$, etc.) is independent of the Riemannian metric, etc.

We conjecture that this invariant coincides with ours. We remark that the validity of this conjecture implies an integrability or rationality theorem of Chern-Simons Perturbation theory.

In [W1] Witten introduced a perturbation of De-Rham complex and Laplace operator using a Morse function. Let us propose to use this perturbation to verify the above conjecture as follows.

Let $G(t ; x, y) \in \operatorname{Hom}\left(\varsigma_{x}, \varsigma_{y}\right) \otimes\left(\bigoplus_{i} \Lambda_{x}^{i} \otimes \Lambda_{y}^{i}\right)$ be the Green kernel of the Laplace operator. Namely if we put

$$
u(t, x)=\int_{y \in M}\langle G(t ; x, y), u(y)\rangle \operatorname{Vol}_{M}
$$


then

$$
\left\{\begin{array}{l}
\lim _{t \rightarrow 0} u(t, x)=u(x) \\
\frac{d u}{d t}=-\Delta u
\end{array} .\right.
$$

In other words $G(t ; x, y)$ is the Schwartz kernel for $e^{-t \Delta}$. We recall the formula

$$
\int_{0}^{\infty} e^{-t \lambda} d t=\lambda^{-1}
$$

Since there is no harmonic form, it follows that

$$
\Delta^{-1}=\int_{0}^{\infty} e^{-t \Delta} d t
$$

We remark that $d^{-1}=\delta \Delta^{-1}$. Therefore we have:

$$
P=(\delta \otimes *) \int_{0}^{\infty} G(t ; x, y) d t .
$$

We put

$$
P(t ; x, y)=(\delta \otimes *) G(t ; x, y) .
$$

We then have

$$
\begin{array}{rl}
\int_{M^{2}} & f d S \operatorname{Tr}_{\Theta}(P(x, y) \wedge P(x, y) \wedge P(x, y)) \\
\quad=\int_{M^{2}} \int_{0}^{\infty} \int_{0}^{\infty} \int_{0}^{\infty} d t_{1} d t_{2} d t_{3} \operatorname{Tr}_{\Theta}\left(P\left(t_{1} ; x, y\right) \wedge P\left(t_{2} ; x, y\right) \wedge P\left(t_{3} ; x, y\right)\right) .
\end{array}
$$

We now try to perturb the Laplace operator using the Morse function $f_{i}$. Let us consider the norm

$$
\langle u, v\rangle_{f_{i}, \varepsilon}=\int_{M}\langle u(x), v(x)\rangle e^{f_{i}(x) / \varepsilon} d S .
$$

Let $\delta_{f_{l}}^{\varepsilon}$ be the dual operator to $d$ with respect to this norm. We put $\Delta_{f_{i}}^{\varepsilon}=\delta_{f_{l}}^{\varepsilon} \circ d$ $+d \circ \delta_{f_{i}}^{\varepsilon}$. Then

$$
\Delta_{f_{i}}^{\varepsilon}=\Delta+\frac{1}{\varepsilon} L_{\varepsilon \operatorname{grad} f_{l}}
$$

Here $L_{X}$ is the Lie derivative by the vector field $X$. We let $G_{\varepsilon, f_{l}}(t ; x, y)$ be the Green function of $\Delta_{f_{i}}^{\varepsilon}$. Using it we define

$$
P_{\varepsilon, f_{i}}(t ; x, y)=\left(\delta_{\varepsilon, f_{i}} \otimes *\right) G_{\varepsilon, f_{i}}(t ; x, y)
$$

and

$$
\int_{M^{2}} d x d y \int_{0}^{\infty} \int_{0}^{\infty} \int_{0}^{\infty} d t_{1} d t_{2} d t_{3} \operatorname{Tr}_{\Theta}\left(P_{\varepsilon, f_{1}}\left(t_{1} ; x, y\right) \wedge P_{\varepsilon, f_{2}}\left(t_{2} ; x, y\right) \wedge P_{\varepsilon, f_{3}}\left(t_{3} ; x, y\right)\right) .
$$

We expect that after suitable normalization this integral also gives the leading term of the 2 loop amplitude of Chern-Simons Perturbation theory.

Let us then see what happens when $\varepsilon$ goes to zero. We remark the leading term of $\Delta_{f_{l}}^{\varepsilon}=\Delta+\frac{1}{\varepsilon} L_{\varepsilon} \operatorname{grad} f_{l}$ is the Lie derivative. Hence after suitable normalization we 
find that $e^{-t\left(\Delta+\frac{1}{\varepsilon} L_{\varepsilon \operatorname{grad} f_{i}}\right)} \approx e^{-\frac{1}{\varepsilon} L_{\varepsilon} \operatorname{grad} f_{i}} \approx\left(\Phi_{f_{i}}^{-t / \varepsilon}\right)^{*}$. Therefore $G_{\varepsilon, f_{i}}(t ; x, y)$ will be a delta measure supported on $M\left(f_{i}\right)$. Thus the limit of (8.4) is an integral of the current supported at the set $M\left(f_{1}\right) \cap M\left(f_{2}\right) \cap M\left(f_{3}\right)$. This shows that this limit is equal to our leading term $Z_{2}\left(M ; f_{1}, f_{2}, f_{3} ; \varsigma\right)$. However to show that our invariant coincides with Chern-Simons Perturbation theory, we need to discuss how the correction term occurs. The author does not know how to do it.

Let us turn to the other topic, that is the relation of our Morse theory invariant to open string theory. (The discussion here is rigorous unless otherwise specified.) For this purpose we consider a domain $D$ with genus 0 and 3 boundaries. Let $\Lambda_{i}, i=1,2,3$ be Lagrangian submanifolds in $T^{*} M$. We choose an almost complex structure $J$ on $T^{*} M$ tamed by the standard symplectic structure. We put

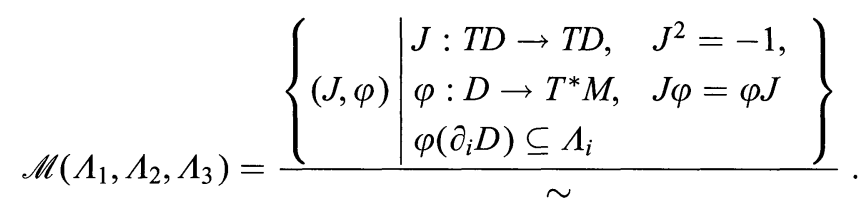

Here $(J, \varphi) \sim\left(\Psi^{*} J, \varphi \circ \Psi\right)$. And $\partial \Lambda=\partial_{1} \Lambda \cup \partial_{2} \Lambda \cup \partial_{3} \Lambda$ is the decomposition to connected components. Now we choose functions $f_{1}, f_{2}, f_{3} \in C^{\infty}(M)$ and put $\Lambda_{i}=$ $\Lambda_{f_{i}}=\left\{\left(x, d f_{i}(x) \mid x \in M\right)\right\}$.

Lemma 8.5. For generic $f_{1}, f_{2}, f_{3} \in C^{\infty}(M)$ the space $\mathscr{M}\left(\Lambda_{f_{1}}, \Lambda_{f_{2}}, \Lambda_{f_{3}}\right)$ is a smooth manifold of dimension 3- $\operatorname{dim} M$. If $M$ is 3-dimensional this space is compact also.

We omit the proof. The main point is that the moduli space of complex structures on $D$ is real 3 dimensional.

Now imitating the proof of [FO] we can prove the following:

Theorem 8.6. If $\varepsilon$ is sufficiently small and $f_{1}, f_{2}, f_{3} \in C^{\infty}(M)$ is generic then $\mathscr{M}\left(\Lambda_{\varepsilon f_{1}}, \Lambda_{\varepsilon f_{2}}, \Lambda_{\varepsilon f_{3}}\right)$ is homeomorphic to $\mathscr{M}_{\Theta}\left(f_{1}-f_{2}, f_{2}-f_{3}, f_{3}-f_{1}\right)$.

We do not prove this theorem here since its proof is completely parallel to [FO].

We next mention some trouble to use this idea to show that the open string on $T^{*} M$ is equivalent to the Morse homotopy of $M$. We remark that in Theorem 8.6 we find $\mathscr{M}_{\Theta}\left(f_{1}-f_{2}, f_{2}-f_{3}, f_{3}-f_{1}\right)$. This space is a bit special compared to the general $\mathscr{M}_{\Theta}\left(g_{1}, g_{2}, g_{3}\right)$. Namely $g_{1}+g_{2}+g_{3}=0$ is satisfied automatically. This causes some trouble for our construction, since we took $\mathscr{M}_{\Theta}\left(\varepsilon_{1} g_{1}, \varepsilon_{2} g_{2}, \varepsilon_{3} g_{3}\right)$ for $\varepsilon_{i}= \pm 1$. The condition $g_{1}+g_{2}+g_{3}=0$ is not preserved by this symmetry. This symmetry was necessary for the cancellation argument.

Namely to cancel the effect from, for example, the degeneration of the second edge, we need to sum up two terms, one from $g_{1}, g_{3}$ and the other from $-g_{1}, g_{3}$. (Here $g_{2}$ can be arbitrary.) So to preserve the relation $g_{1}+g_{2}+g_{3}=0$ we need to put $g_{2} \mapsto g_{2}+2 g_{1}$. This corresponds to the matrix $\left(\begin{array}{ll}1 & 0 \\ 2 & 1\end{array}\right)$. The other two symmetries we need are $\left(\begin{array}{rr}-1 & 0 \\ 0 & 1\end{array}\right)$ and $\left(\begin{array}{ll}1 & 2 \\ 0 & 1\end{array}\right)$. These matrices generate an infinite subgroup of $G L(2 ; \mathbf{Z})$. (The symmetry we used in this paper is a finite group $(\mathbf{Z} / 2 \mathbf{Z})^{3}$.) Then there is trouble to take the average.

There is another trouble. From the point of the open string it is natural to consider the ribbon graph rather than the graph. In the case of the $\Theta$-graph this 
corresponds to consider another Riemann surface as well. Namely we need to take $D^{\prime}=T^{2}-$ Disk. We can try to imitate the construction $\mathscr{M}\left(\Lambda_{1}, \Lambda_{2}, \Lambda_{3}\right)$ and define

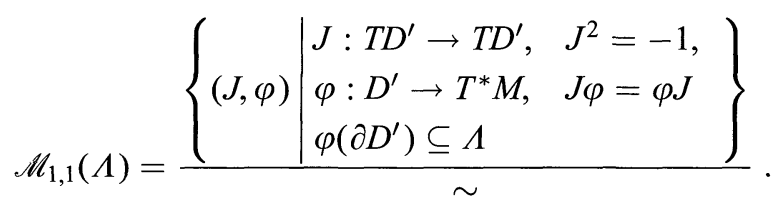

(Here 1,1 in the suffix means that our Riemann surface $D^{\prime}$ is of genus 1 and 1 boundary component. In that sense one we used before is $\mathscr{M}_{0,3}\left(\Lambda_{f_{1}}, \Lambda_{f_{2}}, \Lambda_{f_{3}}\right)$.)

However in fact for this moduli space transversality never holds. In fact we can easily see that elements of $\mathscr{M}_{1,1}(\Lambda)$ are constant maps to the origin. Hence the actual $\operatorname{dimension}$ is $\operatorname{dim} M$, while the formal dimension is $(3-\operatorname{dim} M)(2 g+k-2)$. (Here $g$ is the genus and $k$ is the number of boundary component. In the case of $\mathscr{M}_{1,1}(\Lambda)$ the formal dimension is hence $3-\operatorname{dim} M$.)

This fact is parallel to a similar problem in closed string theory. Namely we consider the moduli space of holomorphic map $\varphi$ from the genus one (or higher) (closed) Riemann surface $\Sigma$ to a symplectic manifold $X$ such that $\varphi^{*}\left(c^{1}(X)\right)=0$, then we have a moduli space homeomorphic to $X$, that is the set of constant maps. The virtual dimension of this moduli space is $2 g(3-\operatorname{dim} M)$ and is different from the actual dimension. This problem was studied by Ruan-Tian [RuT] using the inhomogeneous perturbation. Maybe there is an approach to our open string setting also using inhomogeneous perturbation.

This problem will cause a trouble to generalize Theorem 8.6 also. Namely the homeomorphism in this case should be

$$
\mathscr{M}_{1,1}\left(\Lambda_{\varepsilon f}\right) \stackrel{?}{=} \mathscr{M}_{\Theta}(f-f, f-f, f-f) .
$$

We can not perturb $\mathscr{M}_{\Theta}(f-f, f-f, f-f)=\mathscr{M}_{\Theta}(0,0,0)$ ! to achieve transversality in a similar way discussed in this paper.

The author yet does not know how to overcome these problems, define the open string amplitude and prove that it coincides to our invariant in the case of the cotangent bundle.

Finally we discuss another way to handle the anomaly of the Morse theory version of Chern-Simons perturbation theory. Our argument here is not rigorous, so we put " " in the Lemmas below and mention 8.9 as a conjecture. We recall that the reason we need to take two flat bundles and take the difference is the degeneration of the moduli space parametrized by

$$
\begin{gathered}
R(\vec{f})=\left\{x \in M \mid \operatorname{grad} f_{j}(x) j=1,2,3 \text { are parallel to each other }\right\} \\
R(L)=\{((\vec{f}, g), x) \mid(\vec{f}, g) \in L, x \in R(\vec{f})\} .
\end{gathered}
$$

(See Sect. 2.) Here $L$ is a one dimensional subspace of $\left(C^{\infty}(M)\right)^{3} \times$ Met. We are going to discuss another way to cancel this degeneration. For two functions $f, f^{\prime}$, we consider the space

$$
\bar{S}\left(f, f^{\prime}\right)=\left\{x \in M \mid \operatorname{grad} f(x) \text { is parallel to } \operatorname{grad} f^{\prime}(x)\right\},
$$

and put

$$
S\left(f, f^{\prime}\right)=\bar{S}\left(f, f^{\prime}\right) \cup \bar{S}\left(-f, f^{\prime}\right) .
$$


(We recall that our notation that the two vector is parallel was a bit unusual.) We have:

"Lemma 8.7". For generic $f, f^{\prime}$ the space $S\left(f, f^{\prime}\right)$ is a compact one dimensional oriented manifold.

Proof. The proof that $S\left(f, f^{\prime}\right)$ is one dimensional at a regular point is a counting argument we discussed many times. It is also easy to see that $S\left(f, f^{\prime}\right)$ is smooth outside the critical point of $f$ or $f^{\prime}$. Let us consider a critical point $p$ of $f$. Let $\partial B_{\varepsilon}(p)$ be the boundary of a small metric ball of radius $\varepsilon$ centered at $p$. Then the restriction of grad $f$ to $\partial B_{\varepsilon}(p)$ is regarded as a map from $S^{n-1}$ to $\mathbf{R}^{n} \backslash\{0\}$. The degree of this map is \pm 1 according to the parity of the Morse index. By choosing $\varepsilon$ small grad $f^{\prime}$ is almost constant here. Therefore $\bar{S}\left(f, f^{\prime}\right) \cap \partial B_{\varepsilon}(p)$ and $\bar{S}\left(-f, f^{\prime}\right) \cap \partial B_{\varepsilon}(p)$ both consists of one point. The lemma follows immediately.

Now we consider $S\left(f_{1}, f_{2}\right)$ and $S\left(f_{2}, f_{3}\right)$. For generic $f_{i}$ they intersect only at $\operatorname{Cr}\left(f_{2}\right)$. There is exactly $2^{\# \operatorname{Cr}\left(f_{2}\right)}$ choices to perturb $S\left(f_{1}, f_{2}\right)$ so that it does not intersect to $S\left(f_{2}, f_{3}\right)$. Let $S\left(f_{1}, f_{2} ; \varepsilon\right)$ be those perturbations. We put

$$
L k\left(f_{1}, f_{2} ; f_{2}, f_{3}\right)=2^{-\# \operatorname{Cr}\left(f_{2}\right)} \sum_{\varepsilon} L k\left(S\left(f_{1}, f_{2} ; \varepsilon\right), S\left(f_{2}, f_{3}\right)\right) .
$$

Here $L k$ denotes the linking number. To make it symmetric we take

$$
L k\left(f_{1}, f_{2}, f_{3}\right)=\frac{1}{3}\left(L k\left(f_{1}, f_{2} ; f_{2}, f_{3}\right)+L k\left(f_{2}, f_{3} ; f_{3}, f_{1}\right)+L k\left(f_{3}, f_{1} ; f_{1}, f_{2}\right)\right) .
$$

They we have the following:

“Lemma 8.8". For a generic path L joining $(\vec{f}, g)$ and $\left(\vec{f}^{\prime}, g^{\prime}\right)$ we have

$$
\sum_{\vec{\varepsilon}} \# R(\vec{\varepsilon} L)=4\left(L k\left(\vec{f}^{\prime}\right)-L k(\vec{f})\right) \text {. }
$$

"Proof". The linking number $L k\left(f_{1}, f_{2} ; f_{2}, f_{3}\right)$ changes if and only if $S\left(f_{1}, f_{2}\right)$ intersects with $S\left(f_{2}, f_{3}\right)$ outside $\operatorname{Cr}\left(f_{2}\right)$. This intersection is exactly the point of $R(\vec{f})$. The lemma follows.

These "lemmas" may suggest

Conjecture 8.9. Under the assumption of Main Theorem II, the number $\hat{Z}_{2}\left(f_{1}, f_{2}\right.$, $\left.f_{3}, f_{4} ; \varsigma\right)-\frac{n_{g}}{2} L k\left(f_{1}, f_{2}, f_{3}\right)$ is an invariant of $(M ; \varsigma)$.

\section{References}

[At] Atiyah, M : The geometry and physics of Knots Cambridge: Cambridge University Press, 1990

[AS] Axelrod, S., Singer, I : Chern Simons perturbation theory I In: Proceeding of the XXth DGM Conf. S. Catto and A. Rocha eds, Singapore: World Scientific, 1992, II. J. Diff. Geom. 39, 173-213 (1994)

[BC] Betz, M, Cohen, R.: Graph moduli spaces and Cohomology Operations. Turkish J Math 18, 23-41 (1995)

[Ba] Bar-Natan, D.: Perturbative aspects of the Chern-Simons topological field theory $\mathrm{PhD}$ thesis, Princeton University, 1991 
[Bi] Bismut, J: The Witten complex and the degenerate Morse inequalities $\mathrm{J}$ Diff. Geom 23, 207-240 (1986)

[Ch] Cheeger, J : Analytic torsion and heat equations Ann Math 109, 259-322 (1979)

[F11] Floer, A.: Morse theory for Lagrangian intersection. J Diff. Geom 28, 513-547 (1988)

[F12] Floer, A : Witten's complex and infinite dimensional Morse theory. J Diff Geom 30, 207-221 (1989)

[FU] Freed, D and Uhlenbeck, K : Instanton and four manifolds. MSRI Publication I, Berlin: Springer Verlag, 1974

[Fu1] Fukaya, K : Floer homology for 3-manifolds with boundary In: "Topology Geometry and Field theory". K Fukaya, M. Furuta, T Kohno and K Kotchik, eds., Singapore: World Scientific, 1994, pp 1-23

[Fu2] Fukaya, K : Morse homotopy, $A^{\infty}$ Category and Floer homologies In: The Proceedings of the 1993 GARC Workshop on Geometry and Topology, H.J Kim ed, Seoul National University, pp. 1-102

[Fu3] Fukaya, K : Morse homotopy and its quatization To appear in the Proceeding of Georgia International Conference of Topology

[Fu4] Fukaya, K.: Morse theory and topological field theory To appear in Suukgaku exposition

[FO] Fukaya, K, Oh, Y : 0 loop open string on contangent bundle and Morse homotopy Preprint

[FM] Fulton, W, Macpherson, R: A compactification of configuration space Ann. Math 139, $183-225$ (1994)

[GMM] Guadagnini, E., Martinelli, M., Mintchev, M : Perturvative aspects of Chern Simons field theory Phys Lett., B 227, 111 (1989)

[Kh] Kohno, $\mathrm{T}$ : Invariants of 3-manifolds using representations of mapping class group I Topology 31-2, 203-230 (1992)

[Ko] Kontsevich, M.: Feynman diagram and low dimensional topology. In: "Proceeding of First European Congress of Mathematics" Progress in Mathematics, 120 Birkhäuser, Boston, 97-122 (1994)

[Mü] Müller, W : Analytic torsion and R-torsion of Riemannian manifold Adv Math 28, 233-305 (1978)

[Oh] Oh, Y : Floer cohomology of Lagrangian intersections and Pseudo-holomorphic disks Commun Pure Appl. Math. 46, 949-994 (1993)

[RS] Ray, D, Singer, I : R-torsion and the Laplacian on Riemannian manifolds Adv Math 7, 145-210 (1971)

[ReT] Reshetikhin, N, Turaev, V: Invariants of 3-manifolds via link polynomial and quantum groups. Invent. Math. 103, 547-597 (1991)

[RuT] Ruan, Y, Tian, G.: Mathematical theory of quantum cohomology. Preprint

[Sc] Schwartz, M: Morse homology. Progress in Mathematics 111, Basel: Birkhauser, 1993

[Sm] Smale, S : An infinite dimensional version of Sard's theorem Ann. Math. 87, 213-221 (1973)

[W1] Witten, E : Supersymmetry and Morse theory. J Diff. Geom. 17, 661-692 (1982)

[W2] Witten, E : Quantum field theory and Jones polynomial Commun. Math Phys 121, 351 (1989)

[W3] Witten, E.: Chern-Simons Gauge theory as a string theory. In: "The Floer Memorial Volume", Hofer, Taubes, Winstein, Zehnder, eds., Boston: Birkhäuser, 1995, pp $637-$ 678

Communicated by R.H. Dijkgraaf 